\title{
Scalable Querying of Nested Data
}

\author{
Jaclyn Smith ${ }^{\dagger}$, Michael Benedikt ${ }^{\dagger}$, Milos Nikolic ${ }^{\S}$, Amir Shaikhha $^{\dagger}$ \\ †University of Oxford, UK \\ SUniversity of Edinburgh, UK \\ †name.surname@cs.ox.ac.uk \\ §name.surname@ed.ac.uk
}

\begin{abstract}
While large-scale distributed data processing platforms have become an attractive target for query processing, these systems are problematic for applications that deal with nested collections. Programmers are forced either to perform nontrivial translations of collection programs or to employ automated flattening procedures, both of which lead to performance problems. These challenges only worsen for nested collections with skewed cardinalities, where both handcrafted rewriting and automated flattening are unable to enforce load balancing across partitions.

In this work, we propose a framework that translates a program manipulating nested collections into a set of semantically equivalent shredded queries that can be efficiently evaluated. The framework employs a combination of query compilation techniques, an efficient data representation for nested collections, and automated skew-handling. We provide an extensive experimental evaluation, demonstrating significant improvements provided by the framework in diverse scenarios for nested collection programs.
\end{abstract}

PVLDB Reference Format:

Jaclyn Smith, Michael Benedikt, Milos Nikolic, and Amir Shaikhha. Scalable Querying of Nested Data. PVLDB, 12(xxx): xxxx-yyyy, 2019.

DOI: https://doi.org/10.14778/xxxxxxx.xxxxxxx

\section{INTRODUCTION}

Large-scale, distributed data processing platforms such as Spark 59, Flink 9], and Hadoop 21] have become indispensable tools for modern data analysis. Their wide adoption stems from powerful functional-style APIs that allow programmers to express complex analytical tasks while abstracting distributed resources and data parallelism. These systems use an underlying data model that allows for data to be described as a collection of tuples whose values may themselves be collections. This nested data representation arises naturally in many domains, such as web, biomedicine, and business intelligence. The widespread use of nested data

This work is licensed under the Creative Commons AttributionNonCommercial-NoDerivatives 4.0 International License. To view a copy of this license, visit http://creativecommons.org/licenses/by-nc-nd/4.0/. For any use beyond those covered by this license, obtain permission by emailing info@ vldb.org. Copyright is held by the owner/author(s). Publication rights licensed to the VLDB Endowment.

Proceedings of the VLDB Endowment, Vol. 12, No. xxx

ISSN 2150-8097.

DOI: https://doi.org/10.14778/xxxxxxx.xxxxxxx also contributed to the rise in NoSQL databases [4, 5, 42, where the nested data model is central. Thus, it comes as no surprise that nested data accounts for most large-scale, structured data processing at major web companies 40 .

Despite natively supporting nested data, existing systems fail to harness the full potential of processing nested collections at scale. One implementation difficulty is the discrepancy between the tuple-at-a-time processing of local programs and the bulk processing used in distributed settings. Though maintaining similar APIs, local programs that use Scala collections often require non-trivial and error-prone translations to distributed Spark programs. Beyond programming difficulties, nested data processing requires scaling in the presence of large or skewed inner collections. We next elaborate these difficulties by means of an example.

EXAmPle 1. Consider a variant of the TPC-H database containing a flat relation Part with information about parts and a nested relation COP with information about customers, their orders, and the parts purchased in the orders. The relation COP stores customer names, order dates, part IDs and purchased quantities per order. The type of COP is:

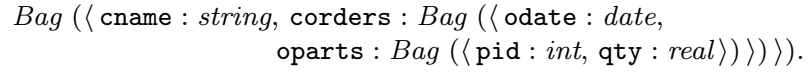

Consider now a high-level collection program that returns for each customer and for each of their orders, the total amount spent per part name. This requires joining COP with Part on pid to get the name and price of each part and then summing up the costs per part name. We can express this using nested relational calculus [11, 56] as:

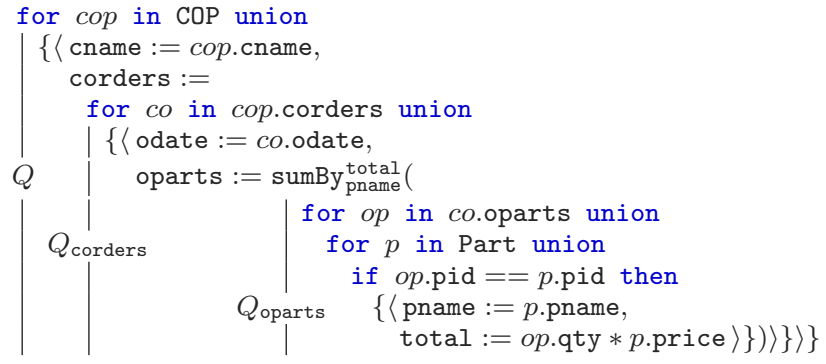

The program navigates to oparts in COP, joins each bag with Part, computes the amount spent per part, and aggregates using sumBy which sums up the total amount spent for each distinct part name. The output type is:

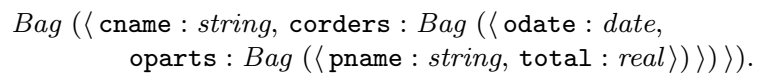


A key motivation for our work is analytics pipelines, which typically consist of a sequence of transformations with the output of one transformation contributing to the input of the next, as above. Although the final output consumed by an end user or application may be flat, the intermediate nested outputs may still be important in themselves: either because the intermediate outputs are used in multiple followup transformations, or because the pipeline is expanded and modified as the data is explored. Our interest is thus in programs consisting of sequence of queries, where both inputs and outputs may be nested.

We next discuss the challenges associated with processing such examples using distributed frameworks.

Challenge 1: Programming mismatch. The translation of collection programs from local to distributed settings is not always straightforward. Distributed processing systems natively distribute collections only at the granularity of top-level tuples and provide no direct support for using nested loops over different distributed collections. To address these limitations, programmers resort to techniques that are either prohibitively expensive or error-prone. In our example, a common error is to try to iterate over Part, which is a collection distributed across worker nodes, from within the map function over COP that navigates to oparts, which are collections local to one worker node. Since Part is distributed across worker nodes, it cannot be referenced inside another distributed collection. Faced with this impossibility, programmers could either replicate Part to each worker node, which can be too expensive, or rewrite to use an explicit join operator, which requires flattening COP to bring pid values needed for the join to the top level. A natural way to flatten COP is by pairing each cname with every tuple from corders and subsequently with every tuple from oparts; however, the resulting (cname, odate, pid, qty) tuples encode incomplete information - e.g., omit customers with no orders - which can eventually cause an incorrect result. Manually rewriting such a computation while preserving its correctness is non-trivial.

Challenge 2: Input and output data representation. Default top-level partitioning of nested data can lead to poor scalability, particularly with few top-level tuples and/or large inner collections. The number of top-level tuples limits the level of parallelism by requiring all data on lower levels to persist on the same node, which is detrimental regardless of the size of these inner collections. Conversely, large inner collections can overload worker nodes and increase data transfer costs, even with larger numbers of top-level tuples. In our example, a small number of customers in COP may lead to poor cluster utilization because few worker nodes process the inner collections of COP.

To cope with limited parallelism, an alternative is to flatten nested data and redistribute processing over more worker nodes. In addition to the programming challenges, unnesting collections also leads to data duplication and consequently redundancy in computation. Wide flattened tuples increase memory pressure and the amount of data shuffled among the worker nodes. Alleviating problems such as disk spillage and load imbalance requires rewriting of programs on an ad hoc basis, without a principled approach.

Representation of nested outputs from transformations is likewise an issue. Flat representations of these outputs can result in significant inefficency in space usage, since parts of the input can appear as subobjects in many places within an output. Exploration of succinctness and efficiency for different representations of nested output has not, to our knowledge, been the topic of significant study.

Challenge 3: Data skew. Last but not least, data skew can significantly degrade performance of large-scale data processing, even when dealing with flat data only. Skew can lead to load imbalance where some workers perform significantly more work than others, prolonging run times on platforms with synchronous execution such as Spark, Flink, and Hadoop. The presence of nested data only exacerbates the problem of load imbalance: inner collections may have skewed cardinalities - e.g., very few customers can have very many orders - and the standard top-level partitioning places each inner collection entirely on a single worker node. Moreover, programmers must ensure that inner collections do not grow large enough to cause disk spill or crash worker nodes due to insufficient memory.

Prior work has addressed some of these challenges. Highlevel scripting languages such as Pig Latin 43] and Scope 13] ease the programming of distributed data processing systems. Apache Hive 6], F1 48, 46], Dremel [40], and BigQuery 47. provide SQL-like languages with extensions for querying nested structured data at scale. Emma 3] and DIQL 27] support for-comprehensions and respectively SQLlike syntax via DSLs deeply-embedded in Scala, and target several distributed processing frameworks. But alleviating the performance problems caused by skew and manually flattening nested collections remains an open problem.

Our approach. To address these challenges and achieve scalable processing of nested data, we advocate an approach that relies on three key aspects:

Query Compilation. When designing programs over nested data, programmers often first conceptualize desired transformations using high-level languages such as nested relational calculus 11, 56, monad comprehension calculus 26], or the collection constructs of functional programming languages (e.g., the Scala collections API). These languages lend themselves naturally to centralized execution, thus making them the weapon of choice for rapid prototyping and testing over small datasets.

To relieve programmers from manually rewriting program for scalable execution (Challenge 1), we propose using a compilation framework that automatically restructures programs to distributed settings. In this process, the framework applies optimizations that are often overlooked by programmers such as column pruning, selection pushdown, and pushing nesting and aggregation operators past joins. These optimizations may significantly cut communication overheads.

Query and Data Shredding. To achieve parallelism beyond top-level records (Challenge 2), we argue for processing over shredded data. In this processing model, nested collections are encoded as flat collections and nested collection queries are translated into relational queries. Early work on nested data 22, 56 proposed a flattening approach in which a nested collection is converted into a single collection containing both top-level attributes, lower-level attributes, and identifiers encoding inner collections. We refer to these as flattened representations below. We will follow later work which considers a normalized representation in which inner collections are stored separately. Regardless of whether flattened or shredded representations are used, it is necessary to rewrite queries over nested data to run over the encoded structure. For example 14 proposes a transformation - 
denoted there as query shredding - which converts nested relational queries to SQL queries that can be run on top of commercial relational processor. Closer to our work is 36 which proposes a shredded data representation and query transformation, applying it to incremental evaluation 36. Both of these works focus on centralized setting.

The motivation for using shredding in distributed settings is twofold. First, a shredded representation of query inputs can enable full parallel processing of large nested collections and their even distribution among worker nodes. The second motivation concerns query outputs. In the setting of complex programs consisting of a sequence of nested-to-nested data transformations, shredding eliminates the need for the reconstruction of intermediate nested results. Compared to flattening, the intermediate outputs are much more succinct. In fact, shredded outputs can even be succinct compared to a native representation, due to the ability to share identifiers for inner bags among distinct outer bags that contain them; we refer to these benefits as the succinct representation used in shredding. The shredded representation also provides more opportunities for reduction of data through aggregation, since aggregates can often be applied locally at a given level of the data. When an aggregation or selection operation on the source object can be translated into a similar operation running on only one component of a shredded representation, then we say that the operator is localized.

In our example, the shredded form of COP consists of one flat top-level collection containing labels in place of inner collections and two dictionaries, one for each level of nesting, associating labels with flat collections. The top-level collection and dictionaries are distributed among worker nodes. We transform the example program to compute over the shredded form of COP and produce shredded output. The first two output levels are those from the shredded input. Computing the last output level requires joining one dictionary of COP with Part, followed by the sum aggregate; both operations can be done in a distributed fashion. In either a flattened or a native representation, the aggregation would be performed over a much larger object, thus resulting in a much larger amount of shuffled data. The shredded output can serve as input to another constituent query in a pipeline. If required downstream, the nested output can be restored by joining together the computed flat collections.

Skew-Resilient Query Processing. To handle data skew (Challenge 3), we propose using different evaluation plans for processing skewed and non-skewed portions of data. We transparently rewrite evaluation plans to avoid partitioning collections on values that appear frequently in the data, thus consequently avoid overloading few worker nodes with significantly more data. Our processing strategy deals with data skew appearing at the top level of distributed collections, but when coupled with the shredding transformation, it can also effectively handle data skew present in inner collections.

In summary, we make the following contributions:

- We propose a compilation framework that transforms declarative programs over nested collections into plans that are executable in distributed environments.

- The framework provides transformations that enable compute over shredded relations; this includes an extension of the symbolic shredding approach, originating in 36, to a larger query language, along with a $m a-$ terialization phase which better suites programs with

$$
\begin{aligned}
& P::=(\operatorname{var} \Leftarrow e)^{*} \\
& e::=\emptyset_{\text {Bag }(F)}|\{e\}| \operatorname{get}(e) \\
& |c| \text { var } \mid \text { e.a } \mid\left\langle a_{1}:=e, \ldots, a_{n}:=e\right\rangle \\
& \text { | for var in } e \text { union } e \mid e \uplus e \\
& \mid \text { let } v a r:=e \text { in } e \mid \text { e PrimOp } e \\
& \mid \text { if cond then } e \mid \operatorname{dedup}(e) \\
& \left|\operatorname{groupBy}_{k e y}(e)\right| \operatorname{sumBy}_{\text {key }}^{\text {value }}(e) \\
& \text { cond }::=\text { e RelOp e } \mid \neg \text { cond } \mid \text { cond BoolOp cond } \\
& T::=S \mid C \\
& C::=\operatorname{Bag}(F) \\
& F::=\left\langle a_{1}: T, \ldots, a_{n}: T\right\rangle \mid S \\
& S::=\text { int } \mid \text { real } \mid \text { string } \mid \text { bool } \mid \text { date } \\
& \text { - Collection Type } \\
& \text { - Flat Type } \\
& \text { - Scalar Type }
\end{aligned}
$$

Figure 1: Syntax of NRC.

nested output to be compiled to parallel programs.

- We introduce techniques that adapt generated plans to the presence of skew.

- We develop a micro-benchmark based on TPC-H and also a benchmark based on a real-word biomedical dataset, suitable for evaluating nesting collection programs. Using these we show that our framework can scale as nesting and skew increase, even when alternative methods are unable to complete at all.

\section{BACKGROUND}

We describe here our source language, which is based on nested relational calculus 11, 56, and our query plan language, based on the intermediate object algebra of [26].

\subsection{Nested Relational Calculus (NRC)}

We support a rich collection programming language NRC as our source language. Programs are sequences of assignments of variables to expressions, where the expression language extends the standard nested relational calculus with primitives for aggregation and deduplication. Figure 1 gives the syntax of NRC. We work with a standard nested data model with typed data items. The NRC types are built up from the basic scalar types (integer, string, etc.), tuple type $\left\langle a_{1}: T_{1} \ldots a_{n}: T_{n}\right\rangle$, and bag type $\operatorname{Bag}(T)$. For ease of presentation, we restrict the bag content $T$ to be either a tuple type or a scalar type, as shown in Figure 1. Set types are modeled as bags with multiplicity one. We refer to a bag of tuples whose each attribute has a scalar type as a flat bag.

$\operatorname{RelOp}$ is a comparison operator on scalars (e.g., $==, \leq$ ), PrimOp is a primitive function on scalars (e.g.,,$+ *$ ), and BoolOp is a boolean operator (e.g., \&\&, \|). A simple term can be a constant, a variable, or an expression e.a. Variables can be free, e.g. representing input objects, or can be introduced in for or let constructs. $\{e\}$ takes the expression $e$ and returns a singleton bag. Conversely, get $(e)$ takes a singleton bag and returns its only element; if $e$ is empty or has more than one element, get returns a default value. $\emptyset_{B a g(F)}$ returns an empty bag.

The if-then-else constructor for expressions of bag type can be expressed using the if-then and union constructors.

$\operatorname{dedup}(e)$ takes the bag $e$ and returns a bag with the same elements, but with all multiplicities changed to one. We im- 
pose a restriction that will be useful in our query shredding methods (Section 4): the input to dedup must be a flat bag.

groupBy $_{k e y}(e)$ groups the tuples of bag $e$ by a collection of attributes key (in the figure we assume a single attribute for simplicity) and for each distinct values of key, produces a bag named GROUP containing the tuples from $e$ with the $k e y$ value, projected on the remaining attributes.

$\operatorname{sumBy}_{\text {key }}^{\text {value }}(e)$ groups the tuples of bag $e$ by the values of their key attributes and for each distinct value of key, sums up the attributes value of the tuples with the key value.

In both groupBy and sumBy operators, we restrict the grouping attributes key to be flat.

\subsection{Plan Language}

Our framework also makes use of a language with algebraic operators, variants of those described in 26 .

Our plan language includes selection $\sigma$, projection $\pi$, join $\bowtie$, and left outer join $\bowtie$, as known from relational algebra.

The unnest operator $\mu^{a}$ takes a nested bag with top-level bag-valued attribute $a$ and pairs each tuple of the outer bag with each tuple of $a$, while projecting away $a$. The outer-unnest operator $\mu^{a}$ is a variant of $\mu^{a}$ that in addition extends each tuple from the outer bag with a unique ID and pairs such tuples with NULL values when $a$ is the empty bag.

The nest operator $\Gamma^{a g g} \begin{gathered}\text { value } \\ \text { key }\end{gathered}$ defines a key-based reduce, parameterized by the aggregate function agg, which could be addition $(+)$ or bag union $(\uplus)$. This operator also casts NULL values introduced by the outer operators into 0 for the + aggregate and the empty bag for the $\uplus$ aggregate.

\section{COMPILATION FRAMEWORK}

Our framework transforms high-level programs into programs optimized for distributed execution. It can generate code operating directly over nested data, as well as code operating over shredded data. Figure 2 shows our architecture.

This section focuses on the standard compilation method, which uses a variant of the unnesting algorithm 26] to transform an input NRC program into an algebraic plan expressed in our plan language. A plan serves as input to the code generator producing executable code for a chosen target platform. For this paper, we consider Apache Spark as the target platform; other platforms such as Apache Flink, Cascading, and Apache Pig could also be supported.

The shredded compilation adds on a shredding phase that transforms an NRC program into a shredded NRC program that operates over flat collections with shredding-specific labels, dictionaries, and dictionary lookup operations. Section 4 describes the shredded data representation and the shredding algorithm. The shredded program then passes through the unnesting and code generation phases, as in the standard method.

Both compilation routes can produce skew-resilient code during code generation. Our skew-resilient processing first uses a lightweight sampling mechanism to detect skew in data. Then, for each operator affected by data skew (e.g., joins), our framework generates different execution strategies that process non-skewed and skewed parts of the data. Section 5 describes our skew-resilient processing technique.

We describe here our standard compilation route for producing distributed programs from high-level queries. It relies on existing techniques from unnesting [26] and codegeneration for distributed processing platforms (e.g., [12]).

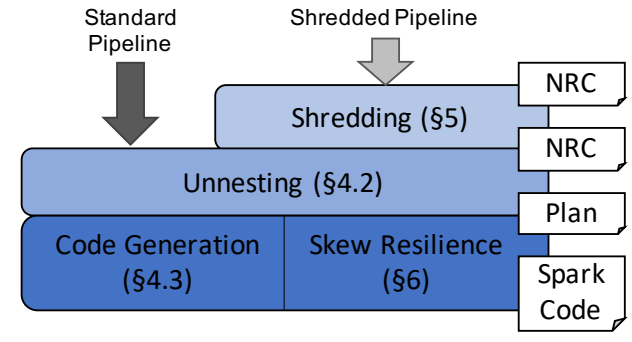

Figure 2: System architecture.

It generates code that automatically inserts IDs and processes NULL values - techniques that play a role in the manually-crafted solutions discussed in the introduction while adding optimizations and guaranteeing correctness. Further, this route forms the basis for our shredded variant and skew-resilient processing module.

\subsection{Unnesting}

The unnesting stage translates each constituent query in an NRC program into a query plan consisting of the operators of the plan language, following the prior work 26], to facilitate code generation for batch processing frameworks.

The unnesting algorithm starts from the outermost level of a query and recursively builds up a subplan subplan ${ }^{e}$ for each nested expression $e$. The algorithm detects joins written as nested loops with equality conditions and also translates each for loop over a bag-typed attribute $x . a$ to an unnest operator $\mu^{a}$.

The algorithm enters a new nesting level when a tuple expression contains a bag expression. When at a non-root level, the algorithm generates the outer counterparts of joins and unnests. At each level, the algorithm maintains a set $\mathcal{G}$ of attributes used as a prefix in the key parameter of any generated nest operator at that level. The set $\mathcal{G}$ is initially empty. Before entering a new nesting level, the algorithm expands $\mathcal{G}$ to include the unique ID attribute and other output attributes from the current level. The $\operatorname{sumBy}_{\text {key }}^{\text {value }}(e)$ operator translates to $\Gamma^{+}$value $_{\mathcal{G} \text {,key }}\left(\right.$ subplan $\left.^{e}\right)$, while $\operatorname{groupBy}_{\text {key }}(e)$ translates to $\Gamma_{\mathcal{G} \text {, key }}^{\uplus \text { value }}\left(\right.$ subplan $\left.^{e}\right)$, where value represents the remaining non-key attributes in $e$. These $\Gamma$ operators connect directly to the subplan built up from unnesting $e$.

The example below details the plans produced by the unnesting algorithm on the running example. We will see that while unnesting addresses the programming mismatch, it highlights the challenges introduced by flattening mentioned in the introduction.

EXAMPle 2. Figure 3 shows a query plan output by an unnesting algorithm for the running example, along with the output type of each operator. For readability, we omit renaming operators from the query plan.

Starting at the bottom left, the plan performs an outerunnest on cop.corders. To facilitate the upcoming nest operation at corders, the outer-unnest associates a unique ID to each tuple cop. The set $\mathcal{G}$ of grouping attributes at this point is $\{$ copID, cname $\}$.

The plan continues with an outer-unnest for co.oparts, extending each tuple $c o$ with a unique ID. The set of grouping attributes is $\{$ copID, coID, cname, odate $\}$. At this point the input is fully flattened. The next operation is an outer join between the plan built up to this point and the Part 


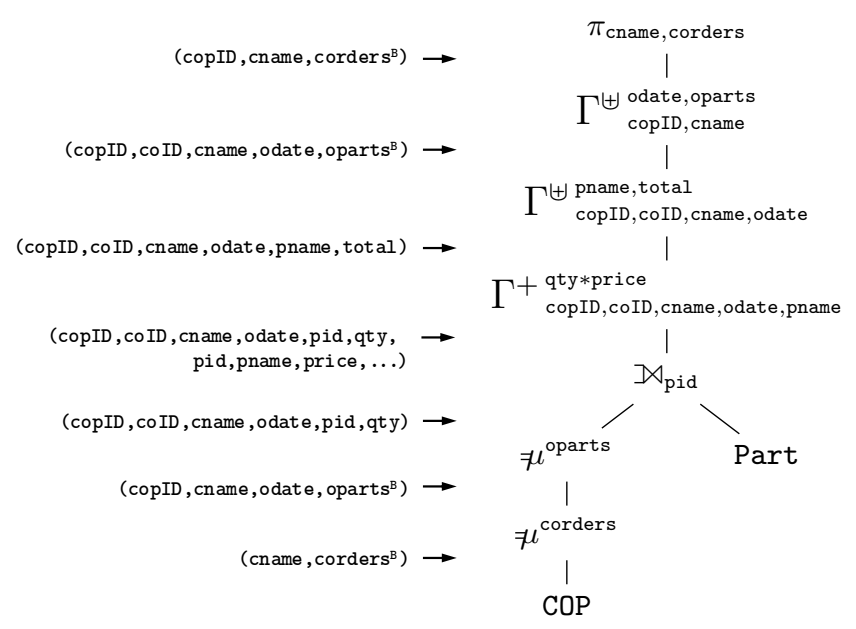

Figure 3: A query plan for the running example with output types. Bag types are annotated with ${ }^{B}$.

relation. This comprises the subplan corresponding to the input for the sumBy expression in the source query.

The sumBy expression is translated to a sum aggregate $\Gamma^{+}$with the key consisting of the grouping attributes from the first two levels and $p$.pname from the key attribute of the sumBy. The second-level subplan continues with a nest operation $\Gamma^{\uplus}$ created from entering the second level at co.oparts with grouping attributes \{copID, coID, cname, odate\}, followed by another nest operation with grouping attributes \{copID, cname $\}$ and a projection on (cname, corders).

The unnesting algorithm may produce suboptimal query plans. We can optimize the plan from Figure 3 by projecting away unused attributes from the Part relation. We can push the sum aggregate $\Gamma^{+}$past the join to compute partial sums of qty values over the output of $\mu^{\text {oparts }}$, grouped by $\{$ copID, coID, cname, odate, pid\}. However, a similar local aggregation over Part brings no benefit since pid is the primary key of Part.

\subsection{Code Generation}

Code generation works bottom-up over a plan produced by the unnesting stage, translating an NRC program into a parallel collection program. For this paper, we describe our compilation in terms of the Spark collection API 59.

We model input/output bags as Spark Datasets, which are strongly-typed, immutable collections of objects; by default, collections are distributed at the granularity of top-level tuples. We translate operators of the plan language into operations over Datasets, taking into account that $\Gamma^{\uplus}$ and $\Gamma^{+}$ also cast NULL values to the empty bag and respectively 0 ; implementation details can be found online 19 .

The design choice to use Datasets was based on an initial set of experiments (Appendix E.1), which revealed that an alternative encoding - using RDDs of case classes - incurs much higher memory and processing overheads. Datasets use encoder objects to operate directly on the compact Tungsten binary format, thus significantly reducing memory footprint and serialization cost. Datasets also allow users to explicitly state which attributes are used in each operation, providing valuable meta-information to the Spark optimizer.

Operators effect the partitioning guarantee ("partitioner" in Spark) of a Dataset. All partitioners are key-based and ensure all values associated with the same key are assigned to the same location. Partitioning guarantees affect the amount of data movement across partitions (shuffling), which can significantly impact the execution cost. Inputs that have not been altered by an operator have no partitioning guarantee. An operator inherits the partitioner from its input and can either preserve, drop, or redefine it for the output. Join and nest operators are the only operators that directly alter partitioning since they require moving values associated with keys to the same partition. These operators control all partitioning in the standard compilation route.

\subsection{Optimization}

The generated plan is subject to standard database optimizations; this includes pushing down selection, projection, and nest operators. Aggregations are pushed down when the key is known to be unique, based on schema information for inputs. When an aggregation is pushed down, the aggregation is only executed locally at each partition. Additional optimizations are applied during code generation; for example, a join followed by a nest operation is directly translated into a cogroup, a Spark primitive that leverages logical grouping to avoid separate grouping and join operations. This is beneficial when building up nested objects with large input bags. These optimizations collectively eliminate unnecessary data as early as possible and thus reduce data transfer during distributed execution.

\section{SHREDDED COMPILATION}

On top of the standard compilation route we build a shredded variant. This shredded compilation route provides a novel approach that begins with an extension of symbolic query shredding from [36], applies a materialization phase that makes the resulting queries appropriate for bulk implementation, optimizes the resulting expressions, and then applies a custom translation into bulk operations.

Our shredded representation encodes a nested bag $b$ whose type $T$ includes bag-valued attributes $a_{1}, \ldots, a_{k}$ by a flat bag $b^{\mathrm{F}}$ of type $T^{\mathrm{F}}$ where each $a_{i}$ has a special type Label and an identifier of a lower-level bag. The representation of $b$ also includes a dictionary tree $b^{\mathrm{D}}$ of type $T^{\mathrm{D}}$ capturing the association between labels and flat bags at each level.

The type $T^{\mathrm{F}}$, always a flat bag type, and the type $T^{\mathrm{D}}$, always a tuple type, are defined recursively. For a tuple type $T=\left\langle a_{1}: T_{1}, \ldots, a_{n}: T_{n}\right\rangle, T^{\mathrm{F}}$ is formed by replacing each attribute $a_{i}$ of bag type with a corresponding attribute of type Label. $T^{\mathrm{D}}$ includes attributes $a_{i}^{\text {fun }}$ and $a_{i}^{\text {child }}$ for each bag-valued $a_{i}$, where $a_{i}^{\text {fun }}$ denotes the dictionary for $a_{i}$ of type Label $\rightarrow B a g\left(T_{i}^{\mathrm{F}}\right)$, while $a_{i}^{\text {child }}$ denotes the dictionary tree of type $T_{i}^{\mathrm{D}}$. Similarly, for a bag type $T=\operatorname{Bag}\left(T_{1}\right)$, we have $T^{\mathrm{F}}=\operatorname{Bag}\left(T_{1}^{\mathrm{F}}\right)$ and $T^{\mathrm{D}}=T_{1}^{\mathrm{D}}$; for scalar types, we have $T^{\mathrm{F}}=T$ and $T^{\mathrm{D}}=\langle\rangle$.

EXAMPLE 3. Recall the type of COP from Example 1 .

$$
\begin{array}{r}
\text { Bag }(\langle\text { cname : string, corders : Bag }(\langle\text { odate : date, } \\
\text { oparts : Bag }(\langle\text { pid : int, qty : real }\rangle)\rangle)\rangle) .
\end{array}
$$

The shredded representation of COP consists of a top-level flat bag $\operatorname{COP}^{\mathrm{F}}$ of type $\mathrm{Bag}(\langle$ cname : string, corders : Label $\rangle)$ 
and a dictionary tree $\mathrm{COP}^{\mathrm{D}}$ of tuple type

$$
\begin{aligned}
& \left\langle\operatorname{corders}^{\text {fun }}: \text { Label } \rightarrow \text { Bag }(\langle\text { odate }: \text { date, oparts }: \text { Label }\rangle),\right. \\
& \text { corders }^{\text {child }}: \text { Bag }(\langle \\
& \quad{ }^{\text {oparts }}{ }^{\text {fun }}: \text { Label } \rightarrow \text { Bag }(\langle\text { pid : string, qty : real }\rangle), \\
& \left.\left.\left.\quad \text { oparts }^{\text {child }}: \operatorname{Bag}(\langle\rangle)\right\rangle\right)\right\rangle
\end{aligned}
$$

The $\mathrm{COP}^{\mathrm{D}}$ dictionary tree encodes the corder $\mathrm{s}^{\text {fun }}$ dictionary for corders labels, the oparts ${ }^{\text {fun }}$ dictionary for oparts labels, and the nesting structure via the child attributes. Since the type system prevents nesting tuples inside tuples, each child dictionary tree is wrapped in a singleton bag.

Note that the shredded representation allows opportunities for sharing in the output, since a given label identifying a nested object may be referenced many times. Appendix D provides a microexperiment that quantifies the benefits of a succinct representation in real-world datasets.

We can convert nested objects to their shredded representations and vice versa. A value shredding function takes as input a nested object $o$ and returns a top-level bag $o^{\mathrm{F}}$ and a dictionary tree $o^{\mathrm{D}}$. This function associates a unique label to each lower-level bag. A value unshredding function performs the opposite conversion [36]. High-level definitions of the shredding and unshredding of a value of type $T$ are straightforward, defined by induction on $T$.

\subsection{Shredded NRC}

Our goal in query shredding is to convert a source NRC program to a program capable of working over shredded representations of input and output.

Shredding approaches. Past implementations of shredding focused on producing a collection of stand-alone subqueries, e.g. in SQL 14, which are well-suited for the parallel execution strategy of relational query engines. Collectively, these subqueries make up the shredded query, with one component query for the top-level bag and one component for each dictionary. To ensure each query can be executed independently, the component query for a dictionary $D$ will need to independently materialize the label domain for $D$; this can result in duplicated computation of different components as well as repetitive iteration steps, which could be problematic for queries with several levels of nesting. We refer to this as monolithic shredding below.

We adopt a sequential approach, which is well-designed for the sequential execution strategy of distributed processing systems. Our method produces a sequence of queries, one for each level of the output, with the query for a given dictionary $D$ depending on the output of its parent. The advantage of this approach is that the labels of $D$ will already be materialized in the parent object, which avoids the duplication of computation and repeated iteration artifacts of monolithic shredding.

We now begin the detailed discussion of our shredding approach, starting with the target languages involved.

As an intermediate stage in shredding, we will make use of a symbolic representation for output dictionaries, defined as partial functions from labels to bags. We will use an intermediate query language, denoted $\mathrm{NRC}^{L b l+\lambda}$, which extends $\mathrm{NRC}$ with a new atomic type for labels and a function type for dictionaries. The grammar of $\mathrm{NRC}^{L b l+\lambda}$ is:

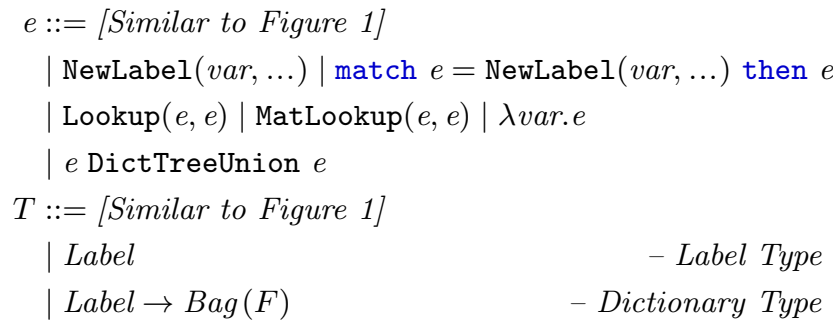

The NewLabel $\left(x_{1}, \ldots, x_{n}\right)$ construct creates a new label encapsulating the values of variables $x_{1}, \ldots, x_{n}$ of flat types. To deconstruct labels we have a "label matching construct":

match $l=\operatorname{NewLabel}(\boldsymbol{x})$ then $F(\boldsymbol{x}, \boldsymbol{y})$

where $F$ is a bag expression, and $\boldsymbol{x}$ and $\boldsymbol{y}$ are tuples of variables. Formally, given any label $l$ and a binding for $\boldsymbol{y}$, we find the unique $\boldsymbol{x}$ such that $l=\operatorname{NewLabel}(\boldsymbol{x})$ and evaluate $F$. If there is no such $\boldsymbol{x}, F$ returns the empty bag. In this expression, $\boldsymbol{x}$ becomes bound although it is free in $F$.

We have the standard $\lambda$ abstraction restricted to label parameters: if $e$ is an expression of type $T$ and $l$ is a label variable, then we can form $\lambda l$.e of type Label $\rightarrow T$. We also have the standard function application: if $e_{1}$ is an expression of type Label $\rightarrow T$ and $e_{2}$ an expression of type Label, then we can form $\operatorname{Lookup}\left(e_{1}, e_{2}\right)$ of type $T$. MatLookup $\left(e_{1}, e_{2}\right)$ corresponds to a lookup of the label returned by $e_{2}$ within the bag of label-bags pairs returned by $e_{1}$. Finally, we have a variation of the binary union for expressions representing dictionary trees, denoted as DictTreeUnion.

\subsection{Query Shredding Algorithm}

A query shredding algorithm takes as input an expression $e$ of type $T$ and produces an expression $e^{\mathrm{F}}$ of type $T^{\mathrm{F}}$ and a dictionary tree $e^{\mathrm{D}}$ of tuple type $T^{\mathrm{D}}$. The algorithm transforms the evaluation problem for $e$ to evaluation problems for $e^{\mathrm{F}}$ and $e^{\mathrm{D}}$ : When the output of $e$ on an input $i$ is $o$, then the outputs of $e^{\mathrm{F}}$ and $e^{\mathrm{D}}$ on the shredded representation of $i$ will be $o^{\mathrm{F}}$ and $o^{\mathrm{D}}$, respectively.

Our shredding transformation consists of two phases. We proceed first with a symbolic query shredding phase, producing succinct expressions manipulating intermediate and output dictionaries defined via $\lambda$ expressions. This phase adapts the work from [36 to our source language. The key to the succinctness of the representation is that the dictionaries are not presented explicitly as a list of label/bag pairs, but rather are over-approximated by $\lambda$-terms that provide general recipes for computing a bag from an arbitrary label.

In the second phase, the shredding transformation is followed by a materialization transformation that produces a sequence of expressions that is free of $\lambda$ abstractions.

Figure 4 shows the shredding transformation in terms of recursive functions $\mathcal{F}$ and $\mathcal{D}$. Given a source expression $e$, the invocation $\mathcal{F}(e)$ returns the expression $e^{\mathrm{F}}$ computing the flat version of the output, while $\mathcal{D}(e)$ returns the dictionary tree $e^{\mathrm{D}}$ corresponding to $e^{\mathrm{F}}$.

The base cases are those of constants and variables: for a scalar $c$, we have $\mathcal{F}(c)=c$ and $\mathcal{D}(c)$ is an empty dictionary tree (line 1); for a variable $x$ of type $T$, the two functions return $x^{\mathrm{F}}$ and $x^{\mathrm{D}}$ whose types $T^{\mathrm{F}}$ and $T^{\mathrm{D}}$ depend on $T$ as described above (line 2).

The interesting cases are those of tuple construction and tuple projection. For each bag-valued attribute $a_{i}$ in a tu- 
ple constructor (line 3$), \mathcal{F}$ replaces the bag expression $e_{i}$ with a new label encapsulating the (flat) free variables of $e_{i}$. The dictionary tree of the tuple constructor includes two attributes: $a_{i}^{\text {fun }}$, which represents the mapping from such a label to the flat variant $e_{i}^{\mathrm{F}}$ of $e_{i}$; and $a_{i}^{\text {child }}$, which represents the dictionary tree for $e_{i}^{\mathrm{F}}$. To conform with the type system, each child dictionary tree is wrapped as a singleton bag as in Example 3. For each scalar-valued attribute $a_{j}$ in a tuple constructor (line 4$), \mathcal{F}$ recurs on the scalar expression $e_{j}$ to produce $e_{j}^{\mathrm{F}}$. Since $e_{j}$ is already flat, $e_{j}^{\mathrm{D}}$ is empty; thus, we omit it from the dictionary tree of the tuple constructor. When accessing a bag-valued attribute $a$ in a tuple expression $e$ (line 5$), \mathcal{F}$ returns a Lookup construct computing the flat bag of $e . a$, based on the dictionary $\mathcal{D}(e) . a^{\text {fun }}$ and label $\mathcal{F}(e) . a$ formed during tuple construction. The returned child dictionary tree serves to dereference any label-valued attributes in the corresponding flat bag. When e. $a$ is a scalar expression (line 6 ), $\mathcal{F}$ recursively computes $e^{\mathrm{F}} . a$, while $\mathcal{D}$ returns an empty dictionary tree.

For the binary union, the same label-valued attribute may correspond to labels that depend on two different sets of free variables. To encapsulate two dictionary trees, the function $\mathcal{D}$ constructs a DictTreeUnion of tuple type (line 11). For the remaining constructs, the functions $\mathcal{F}$ and $\mathcal{D}$ proceed recursively, maintaining the invariant that for any source expression $e$, the free variables of $e^{\mathrm{F}}$ or $e^{\mathrm{D}}$ in the shredded representation correspond to the free variables of $e$.

ExAmple 4. We exhibit the shredding algorithm from Figure 4 on the query $Q$ from Example 1. The algorithm produces a query $Q^{\mathrm{F}}$ computing the top-level flat bag and a query $Q^{\mathrm{D}}$ computing the dictionary tree for $Q^{\mathrm{F}}$. We match $Q$ to the for construct in $\mathcal{F}$ (line 8) and recur to derive $Q^{\mathrm{F}}$ :

for $\operatorname{cop}^{\mathrm{F}}$ in $\mathrm{COP}^{\mathrm{F}}$ union

$\left\{\left\langle\right.\right.$ cname $:=\operatorname{cop}^{\mathrm{F}} \cdot$ cname, corders $\left.\left.:=\operatorname{NewLabel}\left(\operatorname{cop}^{\mathrm{F}}\right)\right\rangle\right\}$

Recall that $\operatorname{COP}^{\mathrm{F}}$ is a flat bag, so $Q^{\mathrm{F}}$ indeed computes a bag of flat tuples. We drop here the unused let binding to $\operatorname{cop}^{\mathrm{D}}$. We derive $Q^{\mathrm{D}}$ after matching corders $:=Q_{\text {corders }}$ in the top-level tuple constructor of $Q$ (line 3):

$$
\begin{aligned}
& \text { let } \operatorname{cop}^{\mathrm{D}}:=\operatorname{COP}^{\mathrm{D}} \text { in } \\
& \left\langle\operatorname{corders}^{\text {fun }}:=\lambda l \text {. match } l=\operatorname{NewLabel}\left(\operatorname{cop}^{\mathrm{F}}\right)\right. \text { then } \\
& \quad \text { for } \operatorname{co}^{\mathrm{F}} \text { in Lookup }\left(\operatorname{cop}^{\mathrm{D}} \cdot \operatorname{corders}, \operatorname{cop}^{\mathrm{F}} \cdot \operatorname{corders}\right) \text { union } \\
& \quad\left\{\left\langle\text { odate }:=\cos ^{\mathrm{F}} \text {.odate, oparts }:=\operatorname{NewLabel}\left(\cos ^{\mathrm{F}}\right)\right\rangle\right\}, \\
& \text { corders } \left.^{\text {child }}:=\left\{Q_{\text {corders }}^{\mathrm{D}}\right\}\right\rangle
\end{aligned}
$$

We omit the unused let binding to $c o^{\mathrm{D}}$ in the expression computing the dictionary corders ${ }^{\text {fun }}$. The query producing the lowest-level dictionary tree $Q_{\text {corders }}^{\mathrm{D}}$ is:

$$
\begin{aligned}
& \text { let } c o^{\mathrm{D}}:=\operatorname{get}\left(\operatorname{cop}^{\mathrm{D}} \cdot \operatorname{corders}^{\mathrm{child}}\right) \text { in } \\
& \left\langle\operatorname{oparts}^{\mathrm{fun}}:=\lambda l \text {. match } l=\operatorname{NewLabel}\left(c o^{\mathrm{F}}\right)\right. \text { then } \\
& \text { sumBy } \text { pname }_{\text {total }}( \\
& \text { for } o p^{\mathrm{F}} \text { in Lookup }\left(c O^{\mathrm{D}} \text {.oparts, } c O^{\mathrm{F}} \text {.oparts }\right) \text { union } \\
& \text { for } p^{\mathrm{F}} \text { in Part } \mathrm{F}^{\mathrm{F}} \text { union } \\
& \text { if }\left(p^{\mathrm{F}} \cdot \mathrm{pid}==o p^{\mathrm{F}} \cdot \mathrm{pid}\right) \text { then } \\
& \left.\left\{\left\langle\text { pname }:=p^{\mathrm{F}} \text {.pname, total }:=o p^{\mathrm{F}} \text {.qty } * p^{\mathrm{F}} \text {.price }\right\rangle\right\}\right), \\
& \left.\operatorname{oparts}^{\text {child }}:=\{\langle\rangle\}\right\rangle
\end{aligned}
$$

Our implementation [19] refines the shredding algorithm to retain only the relevant attributes of free variables in a NewLabel, thus contributing to a succinct representation. For instance, the corders labels in $Q^{\mathrm{F}}$ need only capture the free variables referenced in $c o p^{\mathrm{F}}$.corders and not the $\operatorname{cop}^{\mathrm{F}}$.cname values.

\subsection{Materialization Algorithm}

The symbolic dictionaries produced by the symbolic query shredding algorithm are useful in keeping the inductivelyformed expressions succinct. The second phase of the shredding process, which we refer to as materialization, eliminates $\lambda$ terms in favor of expressions that produce an explicit representation of the shredded output. In the case of a query with flat output, materialization will be a variant of wellknown normalization and conservativity algorithms 44, 26. In the case of nested output, materialization will be the key distinguishing feature of the sequential approach from monolithic shredding. Intuitively, the materialized expression for a given dictionary $D$ will make use of the label domain already materialized for its parent, producing a join of these labels that takes into account the $\lambda$ term associated with $D$ in symbolic shredding.

Our materialization phase produces a sequence of assignments, given a shredded expression and its dictionary tree. For each symbolic dictionary $Q_{i n d e x}^{\mathrm{D}}$ in the dictionary tree, the transformation creates one assignment MATDICT index $_{\text {. }} \Leftarrow$ $e$, where the expression $e$ computes a bag of tuples representing the materialized form of $Q_{\text {index }}^{\mathrm{D}}$. Each assignment can depend on the output of the prior ones. The strategy works downward on the dictionary tree, keeping track of the assigned variable for each symbolic dictionary. Prior to producing each assignment, the transformation rewrites the expression $e$ to replace any symbolic dictionary by its assigned variable and any Lookup on a symbolic dictionary by a MatLookup on its materialized variant.

Materialization needs to resolve the domain of a symbolic dictionary. In our baseline materialization, the expression $e$ computing MATDICT ${ }_{\text {index }}$ takes as input a label domain, the set of labels produced in the parent assignment. The expression $e$ then simply iterates over the label domain and evaluates the symbolic dictionary $Q_{\text {index }}^{\mathrm{D}}$ for each label.

The materialization algorithm from Figure 5 produces a sequence of assignments given a shredded expression, its dictionary tree, and a variable to represent the top-level expression. The MATERIALIZE procedure first replaces the (input) symbolic dictionaries in $e^{\mathrm{F}}$ by their materialized counterparts (line 1) and then assigns this rewritten expression to the provided variable (line 2). Prior to traversing the dictionary tree, its dictionaries are simplified by inlining each let binding produced by the shredding algorithm (line 3).

The MATERIALIzEDicT procedure performs a depth-first traversal of the dictionary tree. For each label-valued attribute $a$, we first emit an assignment computing the set of labels produced in the parent assignment (lines 3-4). We then rewrite the dictionary $a^{\text {fun }}$ to replace all references to symbolic dictionaries, each of them guaranteed to have a matching assignment since the traversal is top-down. We finally produce an assignment computing a bag of label-value tuples representing the materialized form of $a^{\text {fun }}$ (lines 6-8), before recurring to the child dictionary tree (line 9 ).

A technicality is that labels within a label domain can encode values of multiple types, while the expressions above assume that we can match labels coming from each domain against a single known tag $F$, always returning the same type. To solve this we form separate label domains for each tag $\tau$ of a label NewLabe $l_{\tau}$ term. This assures that in each domain, the labels depend upon tuples of the same type.

The ReplaceSymbolicDicts function first recursively inlines all let bindings in the given expression and then per- 


\begin{tabular}{|c|c|c|c|}
\hline & Pattern of $e$ & $\mathcal{F}(e)$ & $\mathcal{D}(e)$ \\
\hline 1 & $c$ & $c$ & \langle\rangle \\
\hline 2 & var & $v a r \mathrm{~F}$ & $\operatorname{var}^{\mathrm{D}}$ \\
\hline 3 & $\left\langle\ldots a_{i}:=e_{i}, \ldots \quad e_{i}\right.$ of bag type & $\left\langle\ldots a_{i}:=\operatorname{NewLabel}\left(\left(x^{\mathrm{F}}\right)_{x \in \operatorname{FrEEVARS}\left(e_{i}\right)}\right), \ldots\right.$ & $\begin{aligned}\langle\ldots & a_{i}^{\text {fun }}:=\lambda l . \text { match } l=\operatorname{NewLabel}(\ldots) \text { then } \mathcal{F}\left(e_{i}\right), \\
& a_{i}^{\text {child }}:=\left\{\mathcal{D}\left(e_{i}\right)\right\}, \ldots\end{aligned}$ \\
\hline & $\ldots a_{j}:=e_{j}, \ldots \quad e_{j}$ of scalar type $\rangle$ & $\left.\ldots a_{j}:=\mathcal{F}\left(e_{j}\right), \ldots\right\rangle$ & $\ldots\rangle \quad / /$ omit empty dictionary tree for $a_{j}$ \\
\hline 5 & e.a of bag type & Lookup $\left(\mathcal{D}(e) \cdot a^{\text {fun }}, \mathcal{F}(e) . a\right)$ & $\operatorname{get}\left(\mathcal{D}(e) \cdot a^{\mathrm{child}}\right)$ \\
\hline 6 & e.a of scalar type & $\mathcal{F}(e) . a$ & \langle\rangle \\
\hline 7 & $\{e\}$ & $\{\mathcal{F}(e)\}$ & $\mathcal{D}(e)$ \\
\hline 8 & for $\operatorname{var}$ in $e_{1}$ union $e_{2}$ & $\begin{array}{l}\text { let } \operatorname{var}^{\mathrm{D}}:=\mathcal{D}\left(e_{1}\right) \text { in } \\
\quad \text { for } \operatorname{var}^{\mathrm{F}} \text { in } \mathcal{F}\left(e_{1}\right) \text { union } \mathcal{F}\left(e_{2}\right)\end{array}$ & let $\operatorname{var}^{\mathrm{D}}:=\mathcal{D}\left(e_{1}\right)$ in $\mathcal{D}\left(e_{2}\right)$ \\
\hline 9 & let $\operatorname{var}:=e_{1}$ in $e_{2}$ & $\begin{array}{l}\text { let } \operatorname{var}^{\mathrm{D}}:=\mathcal{D}\left(e_{1}\right) \text { in } \\
\quad \text { let } \operatorname{var}^{\mathrm{F}} \text { in } \mathcal{F}\left(e_{1}\right) \text { in } \mathcal{F}\left(e_{2}\right)\end{array}$ & let $\operatorname{var}^{\mathrm{D}}:=\mathcal{D}\left(e_{1}\right)$ in $\mathcal{D}\left(e_{2}\right)$ \\
\hline 10 & if cond then $e$ & if $\mathcal{F}($ cond $)$ then $\mathcal{F}(e)$ & $\mathcal{D}(e)$ \\
\hline 11 & $e_{1} \uplus e_{2}$ & $\mathcal{F}\left(e_{1}\right) \uplus \mathcal{F}\left(e_{2}\right)$ & $\mathcal{D}\left(e_{1}\right)$ DictTreeUnion $\mathcal{D}\left(e_{2}\right)$ \\
\hline 12 & $o p(e)$ & $o p(\mathcal{F}(e))$ & $\mathcal{D}(e)$ \\
\hline 13 & $o p\left(e_{1}, e_{2}\right)$ & $o p\left(\mathcal{F}\left(e_{1}\right), \mathcal{F}\left(e_{2}\right)\right)$ & \langle\rangle \\
\hline
\end{tabular}

Figure 4: Query shredding algorithm.

forms the following actions: 1) replaces any Lookup over an input symbolic dictionary with a MatLookup over the corresponding materialized dictionary obtained using the resolver function; 2) $\beta$-reduces any Lookup over an intermediate symbolic dictionary (lambda), returning its body expression with the given label inlined; and 3) replaces any Lookup over a DictTreeUnion with a union of two recursively resolved lookups.

EXAMPle 5. We showcase the MATERIALize procedure on the shredded queries $Q^{\mathrm{F}}$ and $Q^{\mathrm{D}}$ from Example 4 . Let MATCOP, MATCOP corders, and MATCOP corders_oparts denote the materializations of the top-level input bag $\operatorname{COP}^{\mathrm{F}}$ and the two symbolic dictionaries from $\mathrm{COP}^{\mathrm{D}}$, respectively. The procedure replaces $\mathrm{COP}^{F}$ by MATCOP in $Q^{\mathrm{F}}$ (line 1 ), followed by emitting the assignment to the provided variable ToPBAG:

TOPBAG $\Leftarrow$ for $c o p^{\mathrm{F}}$ in MATCOP union

$\left\{\left\langle\right.\right.$ cname $:=\operatorname{cop}^{\mathrm{F}}$. cname, $\left.\operatorname{corders}:=\operatorname{NewLabel}\left(\operatorname{cop} p^{\mathrm{F}}\right)\right\}$

MATERIALIZEDICT produces an assignment computing the set of corders labels from the parent TopBAG (lines 3-4):

LABDoma I $\mathrm{N}_{\text {corders }} \Leftarrow$ $\operatorname{dedup}($ for $x$ in TopBag union $\{\langle$ label $:=x$.corders $\rangle\})$

The function uses the labels from LABDoma I $\mathrm{N}_{\text {corders }}$ to materialize the corders ${ }^{\text {fun }}$ dictionary (lines 6-8):

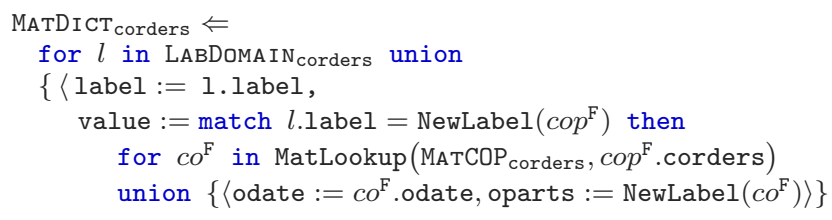

The query computing value corresponds to the body of the corders $^{\text {fun }}$ dictionary, with the Lookup and its symbolic dictionary replaced by their materialized counterparts (line 5).

The function finally recurs on the dictionary tree for oparts, deriving the label domain for oparts ${ }^{\text {fun }}$ from MATDICT corders $_{\text {. }}$

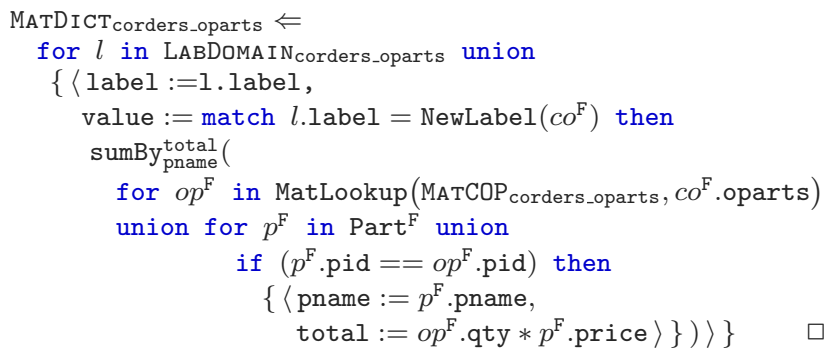

\subsection{Domain Elimination}

In many cases the materialization algorithm produces label domains that are redundant. This represents one major shortcoming of sequential shredding: in some cases making use of labels materialized in the parent is unnecessary.

In order to detect such situations, we optimize our materialization procedure using domain elimination rules.

The first rule recognizes that a child symbolic dictionary is an expression of the form:

$\lambda l$. match $l=\operatorname{NewLabel}(x)$ then

for $y$ in $\operatorname{Lookup}(D, x . a)$ union $e$

where the only used attribute of $x$ is $a$ of label type. We can skip computing the domain of labels for this dictionary and compute the label-value pairs directly from the materialized dictionary MATD corresponding to $D$ :

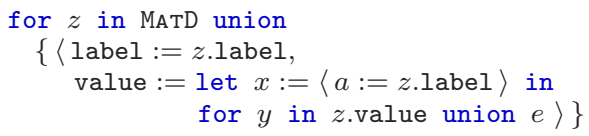

We benefit from this rule when the size of the label domain from the parent assignment is comparable to the size of MATD.

EXAMPLE 6. Returning to Example 5 applying this rule avoids producing LABDOMA I $\mathrm{N}_{\text {corders }}$ as an intermediate result. The materialization of corders ${ }^{\text {fun }}$ now corresponds to:

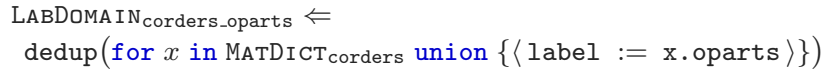


MATDICT corders $\Leftarrow$

for $z$ in MATCOP corders union

$\{\langle$ label := z.label,

value $:=$ for $c O^{\mathrm{F}}$ in $z$.value union

$\left\{\left\langle\right.\right.$ odate $:=c O^{\mathrm{F}}$.odate, oparts $\left.\left.:=\operatorname{NewLabel}\left(c o^{\mathrm{F}}\right)\right\rangle\right\}$

We can extend this rule to match a sumBy construct around the for construct, which would then also enable computing MATDICT corders_oparts $_{\text {directly from MATCOP }}$ corders_oparts.

The second rule for domain elimination recognizes when a label $l$ encodes a non-label attribute $b$ filtering a bag:

$\lambda l$.match $l=\operatorname{NewLabel}(x)$ then

for $y$ in $Y$ union if $(y . a==x . b)$ then $e$

If no other attribute of $x$ appears in $e$, we can produce the label-value pairs from $Y$ using the value of $y . a$ :

$$
\begin{aligned}
& \text { groupBy }_{\text {label }} \text { (for } y \text { in } Y \text { union } \\
& \text { let } x:=\langle b:=y \cdot a\rangle \text { in } \\
& \{\langle\text { label }:=\operatorname{NewLabel}(x) \text {, value }:=e\rangle\})
\end{aligned}
$$

This rule transforms the variable $x$ from free to bound, allowing computing the materialized dictionary from $Y$ only.

\subsection{Partial Shredding}

Shredding can easily be tuned towards either partiallyshredded inputs or partially-shredded outputs.

ExAmple 7. The shredded output in Example 4 in the body of the paper was produced by three queries, the first producing the top-level bag, the second producing the dictionary for corders, and the third producing a dictionary for corders oparts. If there is little or no data skew in oparts, we could decide not to shred corders. The query producing the top-level bag would be unchanged, but we modify the dictionary for corders so that it maps each label to an entire nested object.

$$
\begin{aligned}
& \text { let } c O p^{\mathrm{D}}:=\mathrm{COP}^{\mathrm{D}} \text { in } \\
& \left\langle\operatorname{corders}^{\text {fun }}:=\lambda l \text {. match } l=\operatorname{NewLabel}\left(\operatorname{cop}^{\mathrm{F}}\right)\right. \text { then } \\
& \text { for } c o^{\mathrm{F}} \text { in Lookup }\left(c o p^{\mathrm{D}} \text {. corders, } c o p^{\mathrm{F}}\right. \text {.corders) union } \\
& \left\{\left\langle\text { odate }:=c O^{\mathrm{F}} \text {.odate, oparts }:=\operatorname{sumBy}_{\text {pname }}^{\text {total }}(\right.\right. \\
& \text { for } o p \text { in } c o^{\mathrm{F}} \text {.oparts union } \\
& \text { for } p^{\mathrm{F}} \text { in Part }{ }^{\mathrm{F}} \text { union } \\
& \text { if } p^{\mathrm{F}} \text {.pid }==o p \text {.pid then } \\
& \left\{\left\langle\text { pname }:=p^{\mathrm{F}} \cdot\right.\right. \text { pname, } \\
& \text { total } \left.\left.:=o p \cdot \text {.qty } * p^{\mathrm{F}} \cdot \text { price }\right\rangle\right\} \\
& )\rangle\} \\
& \text { corders } \left.^{\text {child }}:=\{\langle\rangle\}\right\rangle
\end{aligned}
$$

The modification over the fully-shredded version is to replace a call to the NewLabel function at oparts with the inlining of the oparts subquery.

\subsection{Extensions for Shredded Compilation}

Given that the output of materialization is an NRC program with expressions containing only a subset of $\mathrm{NRC}^{L b l+\lambda}$, there are only a few extensions required to support compilation for shredded data. Dictionaries are simply considered to be a special instance of a bag, keyed with values of a label type and supporting lookup operations. A MatLookup is translated directly into an outer join in the plan language, providing opportunities for pushed aggregation and merging with nest operators to form cogroups in the code generated for value unshredding.

The shredded representation allows nested operations in the input query to be translated to operations working only
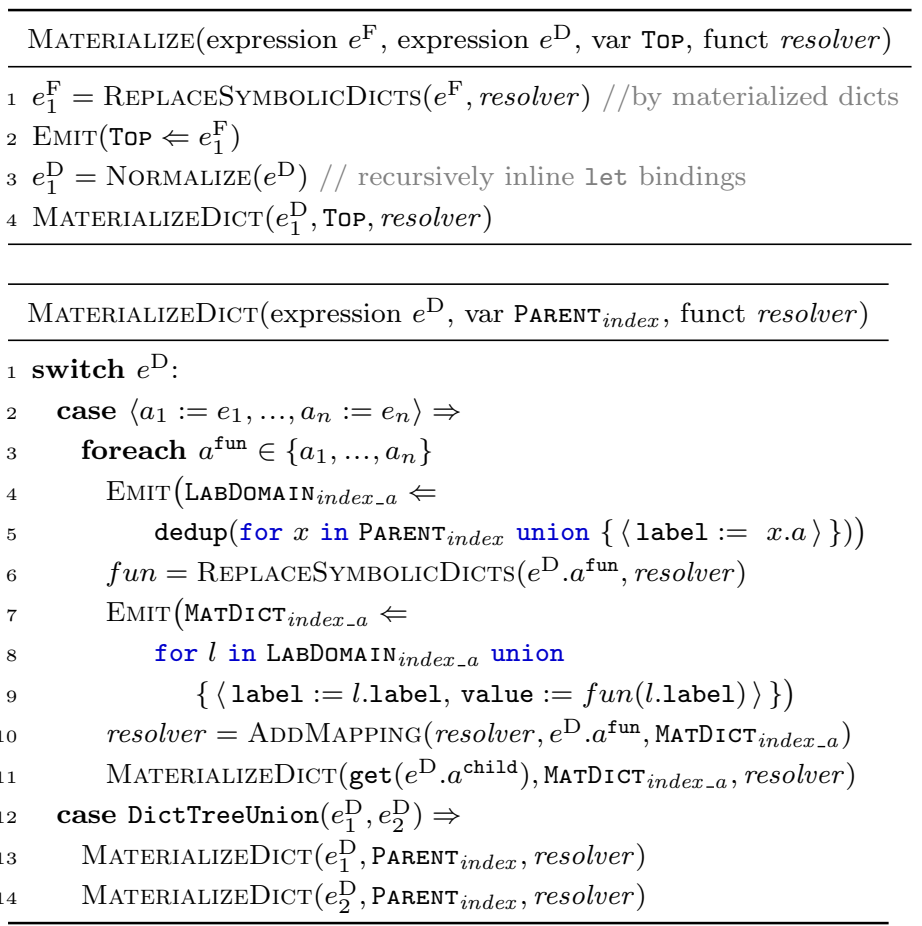

Figure 5: Materialization algorithms.

on the dictionaries relevant to that level, these are the localized operations first discussed in the introduction. For example, the sumBy in the running example will operate directly over the oparts dictionary. This produces a plan, similar to a subset of the plan in Figure 3 , that has isolated the join and aggregate operation to the lowest level dictionary. This avoids flattening the input via the series of unnest operators and avoids the programming mismatch also described in Section 1 .

The label-bag representation of dictionaries can lead to overheads when we want to join with a bag value. Thus, a relational dictionary representation of label-value pairs can be useful for implementation. To provide flexibility in dictionary representation, we introduce an operator BagToDict, which casts a bag to a dictionary while delaying explicit representation until the relevant stage of compilation.

For our current code generation, dictionaries are represented the same as bags, Dataset[T] where $\mathrm{T}$ contains a label column (label) as key. Top-level bags that have not been altered by an operator have no partitioning guarantee and are distributed by the same default strategy as bags in the standard compilation route. Dictionaries have a label-based partitioning guarantee, which is the key-based partitioning guarantee described in Section 3.2 where all values associated to the same label reside on the same partition.

\section{SKEW-RESILIENT PROCESSING}

We provide a novel approach to skew handling, which adapts a generated plan based on statistics of the data at runtime and supports shredding. The framework up to this point has not addressed skew-related bottlenecks. Skew is a consequence of key-based partitioning where all values with the same key are sent to the same partition. If the collected values of a key $\left(k e y_{1}\right)$ are much larger than the collected values of another key $\left(k e y_{2}\right)$, then the partition housing $k e y_{1}$ 
values will take longer to compute than others. We refer to such keys as heavy, and all others as light. In the worst case, the partition is completely saturated and the evaluation is heavily burdened by reading and writing to disk. We mitigate the effects of skew by implementing skew-aware plan operators that automate the skew-handling process.

The identification of heavy keys is central to automating the skew-handling process. We use a sampling procedure to determine heavy keys in a distributed bag, considering a key heavy when at least a certain threshold of the sampled tuples $(10 \%)$ in a partition are associated with that key; in our experiments, we use the threshold of $2.5 \%$. Based on the heavy keys, the input bag is split into a light component and a heavy component. We refer to these three components (light bag, heavy bag, and heavy keys) as a skew-triple.

A skew-aware operator is a plan operator that accepts and returns a skew-triple. If the set of heavy keys is not known, then the light and heavy components are unioned and a heavy key set is generated. This set of heavy keys remains associated to that skew-triple until the operator does something to alter the key, such as a join operation.

In essence, the skew-aware plan consists of two plans, one that works on the light component (light plan), and one that works on the heavy component (heavy plan). The light plan follows the standard implementation for all operators. The light plan ensures that values corresponding to light keys reside in the same partition. The heavy plan works on the heavy input following an alternative implementation. The heavy plan ensures that the values associated to heavy keys are distributed across partitions. This ability to preserve the partitioning guarantee of light keys and ensure the distribution of large collections associated to heavy keys enables skew-resilient processing.

Figure 6 provides the implementation of the main skewaware operations. The skew-aware operators assume that the set of heavy keys is small. The threshold used to compute heavy keys puts an upper bound on their number; for example, the threshold of $2.5 \%$ means there can be at most $\frac{100}{2.5}=40$ distinct heavy keys in the sampled tuples per partition. This small domain allows for lightweight broadcasting of heavy keys and, in the case of the skew-aware join operations, the heavy values of the smaller relation. Broadcast operations maintain skew-resilience by ensuring the heavy key values in the larger relation remain at their current location and are not consolidated to the same node.

All nest operations merge the light and heavy components and follow the standard implementation, returning a skewtriple with an empty heavy component and a null set of heavy keys. Aggregation $\Gamma^{+}$mitigates skew-effects by default by reducing the values of all keys. A grouping operation $\Gamma^{\uplus}$ cannot avoid skew-related bottlenecks. More importantly, skew-handling for nested output types would be detrimental to our design - attempting to solve a problem that the shredded representation already handles gracefully.

The encoding of dictionaries as flat bags means that their distribution is skew-resilient by default. In the shredded variant, input dictionaries come as skew triples, with known sets of heavy labels. The skew-aware evaluation of a dictionary involves casting of a bag with the skew-aware BagToDict operation. This maintains the skew-resilience of dictionaries by repartitioning only light labels, and leaving the heavy labels in their current location, as shown in Figure 6 .

\begin{tabular}{|c|c|}
\hline Plan Operator & Definition \\
\hline$X \bowtie_{f(x)=g(y)} Y$ & 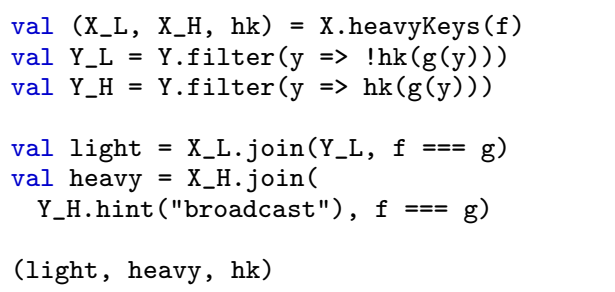 \\
\hline$\Gamma^{a g g} \underset{\text { key }}{\text { value }} X$ & $\begin{array}{l}\text { val unioned }=\mathrm{X}_{-} \mathrm{L} \text {.union }\left(\mathrm{X}_{-} \mathrm{H}\right) \\
/ / \text { proceed with light plan } \\
\text { val light }=\ldots \\
\text { (light, sc.emptyDataset, null) }\end{array}$ \\
\hline BagToDict $X$ & $\begin{array}{l}\text { val }\left(X_{-} L, X_{-} H, h k\right)= \\
X \cdot \text { heavyKeys }(x=x \cdot l a b e l) \\
\text { val light }=X_{-} L \cdot \text { repartition }(x=>x \cdot \text { label }) \\
\text { val heavy }=X_{-} H \\
\text { (light, heavy, hk) }\end{array}$ \\
\hline
\end{tabular}

Figure 6: Skew-aware implementation for the plan language operators using Spark Datasets.

\section{EXPERIMENTS}

We evaluate the performance of the standard and shredded variants of our framework and compare against existing systems that support nested data processing for both generated and real-world datasets. One goal of the experiments is to evaluate how the succinct dictionary representation of shredding compares to the flattening methods. The following experiments look at how the strategies scale with increased levels of nesting, number of top-level tuples, and the size of inner collections. We explore how aggregations can reduce dictionaries and downstream dictionary operations for nested and flat output types. Finally, to highlight our ability to handle skewed data, we evaluate the performance of our framework for increasing amounts of skew.

Our experimental results can be summarized as follows:

- For flat-to-nested queries, the shredded variant adds no overhead when producing nested results and shows up to $25 \mathrm{x}$ gain when producing shredded results.

- For nested input, the shredded compilation route has a 16x improvement for shallow nesting and scales to deeper levels which flattening methods cannot handle.

- The shredded data representation provides more opportunities for optimizations in nested-to-flat queries with aggregation, providing a $6 \mathrm{x}$ improvement over the flattening methods.

- The skew-aware shredded compilation route outperforms flattening methods with $15 \mathrm{x}$ improvement in runtime and $33 \mathrm{x}$ reduction in shuffling for moderate skew, as well as graceful handling of increasing amounts of skew while the flattening methods, skew-aware and skew-unaware, are unable to complete at all.

Full details of the experimental evaluation can be found in the Appendix. Framework and experimental implementation can be found in our github repository 19 .

Experimental environment. Experiments were run on a Spark 2.4.2 cluster (Scala 2.12, Hadoop 2.7) with five 


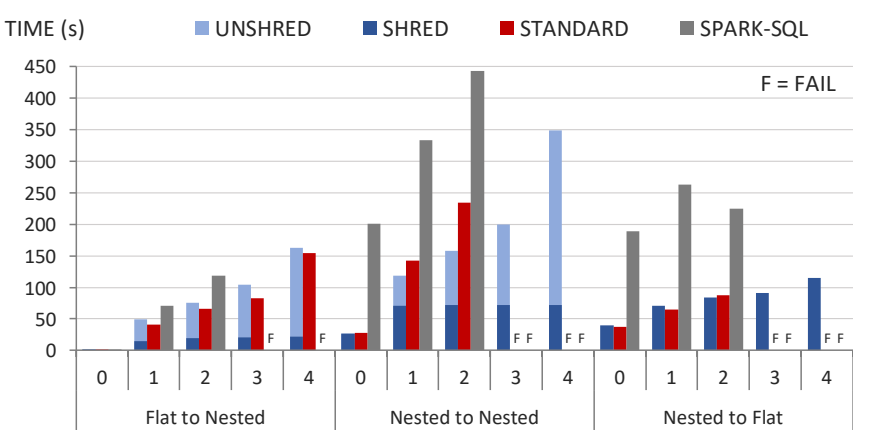

(a) Narrow schema

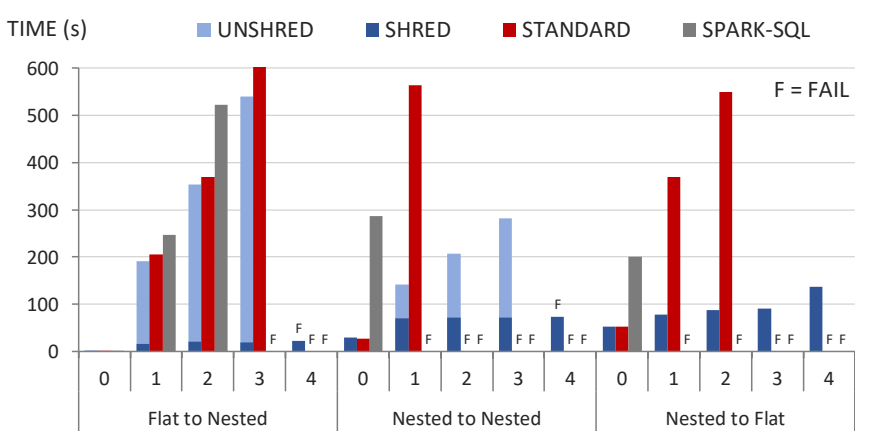

(b) Wide schema

Figure 7: Performance comparison of the narrow and wide benchmarked TPC-H queries with varying levels of nesting (0-4).

workers, each with 20 cores and 320G memory. Each experiment was run as a Spark application with 25 executors, 4 cores and $64 \mathrm{G}$ memory per executor, $32 \mathrm{G}$ memory allocated to the driver, and 1000 partitions used for shuffling data. Broadcast operations are deferred to Spark, which broadcasts anything under 10MB. Total reported runtime starts after caching all inputs. We provide summary information on shuffling cost - full details of which can be found in the github repository. All missing values correspond to a run that crashed due to memory saturation of a node.

Benchmarks. We create two NRC query benchmarks; a micro-benchmark based on the standard TPC-H schema and the second based on biomedical datasets from the International Cancer Genome Consortium (ICGC) 34.

The TPC-H benchmark contains a suite of flat-to-nested, nested-to-nested, and nested-to-flat queries - all with 0 to 4 levels of nesting and organized such that the number of top-level tuples decrease as the level of nesting increases. All queries start with the Lineitem table at level 0 , then group across Orders, Customer, Nation, then Region, as the level increases. Each query has a wide variant where we keep all the attributes, and a narrow variant which follows the grouping with a projection at each level. For scale factor 100 , this organization gives query results with 600 million, 150 million, 15 million, 25, and 5 top-level tuples. Flat-tonested queries perform the iterative grouping above to the relational inputs, returning a nested output. At the lowest level we keep the partkey and quantity of a Lineitem. At the higher levels the narrow variant keeps only a single attribute, e.g., order_date for Orders, customer_name for Customer, etc. The nested-to-nested queries take the materialized result of the flat-to-nested queries as input and perform a join with Part at the lowest level, followed by sumBy pname pty $^{\text {qtyce }}$, as in Example 1. The nested-to-nested queries thus produce the same hierarchy as the flat-to-nested queries. Finally, the nested-to-flat queries follow the same construction as the nested-to-nested queries, but apply sumBy ${ }_{\text {name }}^{\text {qty } * \text { price }}$ at toplevel, where name is one of the top-level attributes; this returns a flat collection persisting only attributes from the outermost level. We use the skewed TPC-H data generator 53 to generate $100 \mathrm{~GB}$ datasets with a Zipfian distribution; skew factor 4 is the greatest amount of skew, with a few heavy keys occurring at a high frequency. Non-skewed data is generated with skew factor 0 , generating keys at a normal distribution as in the standard TPC-H generator.

The biomedical benchmark includes an end-to-end pipeline E2E, based on 60, consisting of 5 steps. Matching one of the major motivations of this work, results of the intermediate steps produce output that has significant nesting.

The inputs include a two-level nested relation $\mathrm{BN}_{2}(280 \mathrm{~GB})$ 34, 38, a one-level nested relation $\mathrm{BN}_{1}$ (4GB) 52, and five relational inputs - the most notable of which are $\mathrm{BF}_{1}(23 \mathrm{G})$, $\mathrm{BF}_{2}$ (34GB), and $\mathrm{BF}_{3}$ (5KB) 34, 23. The first two steps of $\mathrm{E} 2 \mathrm{E}$ are the most expensive. STEP 1 flattens the whole of $\mathrm{BN}_{2}$, while performing a nested join on each level $\left(\mathrm{BF}_{2}\right.$ at level 1 and $\mathrm{BF}_{3}$ at level 2), aggregating the result, and finally grouping to produce nested output. $\mathrm{STEP}_{2}$ joins and aggregates $\mathrm{BN}_{1}$ on the first-level of the output of $\mathrm{STEP}_{1}$. Information on the additional steps and additional queries of the biomedical benchmark can be found in Appendix C.

Evaluation strategies and competitors. We compare the standard compilation route (Section 3) to the shredded compilation route (Section 4). On each of our benchmarks, we have also compared to a wide array of external competitors: an implementation via encoding in SparkSQL 7]; Citus, a distributed version of Postgres [17]; MongoDB [42], and the recently-developed nested relational engine DIQL 25. Since we found that SparkSQL outperformed the other competitors, we include only SparkSQL in the body of the paper, deferring discussion of the others to the github repository. SparkSQL does not support explode (i.e., UNNEST) operations in the SELECT clause, requiring the operator to be kept with the source relation which forces flattening for queries that take nested input. The SparkSQL queries were manually written based on this restriction. STANDARD denotes the standard compilation route and produces results that match the type of the input query. STANDARD also serves as a means to explore the general functionality of flattening methods. SHRED denotes the shredded compilation route with domain elimination, leaving its output in shredded form. UNSHRED represents the time required to unshred the materialized dictionaries, returning results that match the type of the input query. UNSHRED is often considered in combination with SHRED to denote total runtime of the shredded compilation route when the output type is nested. SHRED assumes a downstream operation can consume materialized dictionaries. The above strategies do not implement skew-handling; we use $\mathrm{STANDARD}_{\mathrm{SKEW}}, \mathrm{SHRED}_{\mathrm{SKEW}}$, and SHRED $_{\text {SKEW }}^{+\mathrm{U}}$ to denote the skew-handling variations. When executing queries, we use the optimal plan for a given strategy, which at a minimum includes pushing projections and domain-elimination (Section 4.4) any further optimizations are described within the context of each experiment.

Flat-to-nested queries for non-skewed data. The 
TPC-H flat-to-nested queries are used to explore building nested structures from flat inputs when the data is not skewed. Figure 7 shows the running times of SparkSQL, Standard , Shred, and Unshred for all levels of nesting.

SHRED runs to completion for all levels, remaining constant after the first level and exhibiting nearly identical runtimes and max data shuffle for narrow and wide. UNSHRED and STANDARD have comparable runtimes overall and a max data shuffle that is 20x that of SHRED. For deeper levels of nesting, these methods fail to store the local nested collections when tuples are wide. The use of cogroup in these methods (Section 3.2 provides performance advantages for shallow nesting in comparison to SparkSQL, which is unable to scale for the small number of top-level tuples that occur at deeper levels of nesting even for narrow tuples. SHRED shows $6 \mathrm{x}$ improvement for narrow queries and 26x for wide queries. The flat-to-nested case introduces a worst case for the shredded compilation route, where the shredded query is executed only to group everything up in the unshredding phase. Even in this case, the shredded compilation route adds no additional overhead in comparison to flattening.

Nested-to-nested queries for non-skewed data. The TPC-H nested-to-nested queries are used to explore the effects of aggregation on non-skewed data. We use the wide version of the flat-to-nested queries as input to evaluate the impact of projections on nested input. Figure 7 shows the runtimes of SparkSQL, Standard , Shred, and Unshred for increasing levels of nesting. SparkSQL exhibits consistently worse performance while maintaining the same total amount of shuffled memory as STANDARD . This behavior starts for 0 levels of nesting, suggesting overheads related to pushing projections that may be compounded when combined with the explode operator at deeper levels of nesting. SparkSQL does not survive even for one-level of nesting for wide tuples. The rest of the methods diverge with respect to runtime at one level of nesting, with SHRED exhibiting the best performance overall.

For wide queries STANDARD is burdened by the top-level attributes that must be included in the sumBy pname $\mathrm{qty}$ *price aggregate operation, and only finishes for one level of nesting. In the narrow case, STANDARD survives the aggregation but fails for the small number of top-level tuples in the deeper levels of nesting. UNSHRED shows a linear drop in performance as the number of top-level tuples decrease from 150 million to 25 tuples, but at a much lower rate compared to the flattening methods. With 5 top-level tuples, UNSHRED suffers from a poor distribution strategy that must maintain the wide tuples in nested local collections, and crashes due to memory saturation. Overall, SHRED and UNSHRED show up to a $8 \mathrm{x}$ performance advantage for shallow levels of nesting and exhibit $3 x$ less total shuffle. Further, in comparison to the previous results without aggregation, the localized aggregation produces up to a $3 \mathrm{x}$ performance gain in unshredding. This experiment highlights how the succinct representation of dictionaries is a key factor in achieving scalability. The use of this succinct representation enables localized aggregation (Section 4.6) which avoids data duplication, reduces the size of dictionaries, and lowers unshredding costs to support processing at deeper levels of nesting even when the output to be returned is nested.

Nested-to-flat with non-skewed inputs. We use nestedto-flat queries to explore effects of aggregation for queries that navigate over multiple levels of the input; such queries

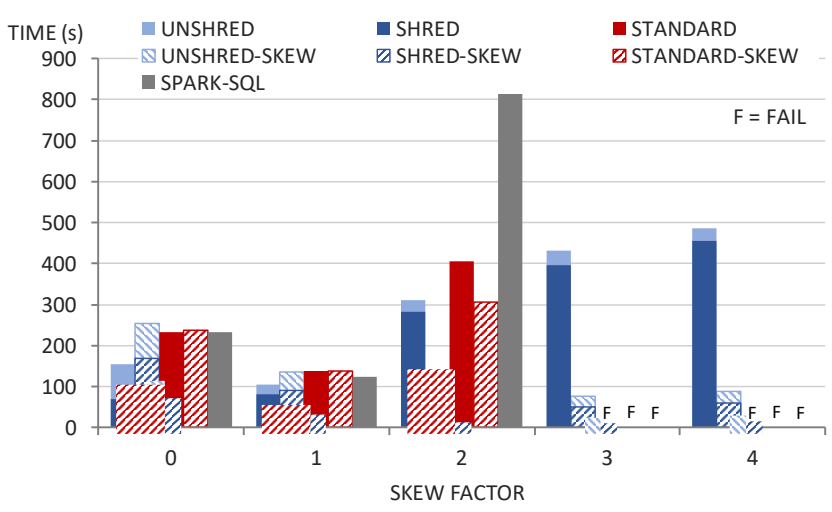

Figure 8: Nested-to-nested narrow TPC-H query with two levels of nesting on increasingly skewed datasets.

are challenging for shredding-based approaches due to an increase in the number of joins in the query plan. Figure 7 shows the runtimes for SparkSQL, StANDARD and SHrED, where the unshredding cost for flat outputs is zero. Similar to the nested-to-nested results, STANDARD is unable to run for the small number of top-level tuples associated with deeper levels of nesting for both the narrow and wide case. SparkSQL has the worst performance overall, and cannot complete for even one level of nesting when tuples are wide.

SHRED outperforms STANDARD with a 6x runtime advantage, over a $2 \mathrm{x}$ total shuffle advantage for wide queries, and runs to completion even when STANDARD fails to perform at all. At two levels of nesting, the execution of SHRED begins with a localized join between the lowest-level Lineitem dictionary and Part, then aggregates the result. Unlike the flattening procedures, these operations avoid carrying redundant information from top-level tuples, thereby reducing the lowest-level dictionary as much as possible. The evaluation continues by dropping all non-label attributes from the first-level Order dictionary, which reduces the first-level dictionary and results in a cheap join operation to reassociate the next two levels. This benefit persists even as the number of intermediate lookups increase for deeper levels of nesting, exhibiting resilience to the number of top-level tuples.

Due to the nature of the non-skewed TPC-H data, STANDARD does not benefit from local aggregation (before unnesting) and also does not benefit from pushing the aggregation past the nested join since the top-level information must be included in the aggregation key. These results show how the shredded representation can introduce more opportunities for optimization in comparison to traditional flattening methods, supporting more cases where pushed and localized operations can be beneficial.

Skew-handling. We use the narrow variant of the nestedto-nested queries with two levels of nesting to evaluate our skew-handling procedure. We use the materialized flat-tonested narrow query (COP) as input.

The TPC-H data generator produces skew by duplicating values; thus, pushing aggregations will reduce the duplicated values associated to a heavy key thereby diminishing skew. When designing this experiment, we found that skewunaware methods benefit from aggregation pushing, whereas skew-aware methods benefit more from maintaining the distribution of heavy keys (skew-resilience, Section 5). Figure 8 shows the runtimes for all methods for increasing amounts of skew. Skew-unaware methods are reported with pushed aggregation and skew-aware methods without pushed aggre- 


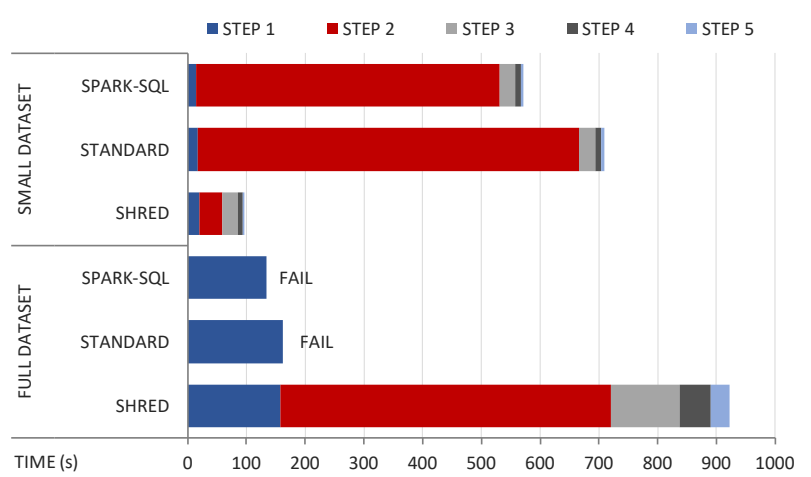

Figure 9: End-to-end pipeline of the biomedical benchmark.

gation. Appendix E.6 provides results without aggregation pushing for all methods, which suggests further benefits to skew-aware methods in the case where aggregation pushing does not reduce skew.

The results in Figure 8 show that SHRED $_{\text {SKEW }}$ has up to a 15x performance gain over other methods for moderate amounts of skew (skew-factor 2). The skew join of SHRED shuffles up to $33 \mathrm{x}$ less for moderate skew and up to $74 \mathrm{x}$ less for high skew than the skew-unaware join of SHRED, which has also first performed aggregation pushing. At greater amounts of skew (skew-factor 3 and 4), SparkSQL, STAN-

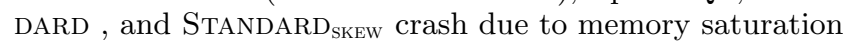
from flattening skewed inner collections.

The performance gain of the skew-aware shredded compilation route over the skew-aware standard compilation route is attributed to the succinct representation of dictionaries, which provides better support for the skew-aware operators. The shredded compilation route, regardless of skewhandling, runs to completion for all skew factors. Beyond supporting the distribution of the large-skewed inner collections, the localized aggregation reduces the size of the lowerlevel dictionary thereby decreasing the skew. This highlights how the shredded representation is able to handle skew even before the skew-handling procedure is applied. Overall, we see that the skew-aware components ads additional benefits by maintaining skew-resilience and reducing the amount of data shuffle required to handle heavy keys.

Biomedical Benchmark. Figure 9 shows the runtimes for SparkSQL, STD, and SHrEd for the E2E pipeline of the biomedical benchmark. The final output of E2E is flat, so no unshredding is needed. SHrED exhibits many advantages, overall surviving the whole pipeline. Since STANDARD and SparkSQL fail during $\mathrm{STEP}_{2}$, we also provide results with a smaller dataset using only $6 \mathrm{~GB} \mathrm{BN}_{2}, 2 \mathrm{~GB} \mathrm{BF}_{1}$, and $4 \mathrm{~GB} \mathrm{BF}_{2}$. The results for $\mathrm{STEP}_{1}$ using the small and full dataset exhibit similar behavior to the nested-to-flat results for TPC-H; given that the whole $\mathrm{BN}_{2}$ input is flattened and there are many projections, STANDARD and SparkSQL are not too burdened by executing nested joins via flattening.

The methods diverge at $\mathrm{STEP}_{2}$, where a nested join between $\mathrm{BN}_{1}$ and the output of $\mathrm{STEP}_{1}$ leads to an explosion in the amount of data being shuffled. SHRED displays up to a 16x performance gain for $\mathrm{STEP}_{2}$ with the small dataset. Despite the small number of output attributes, the result of the join between flattened output of $\mathrm{STEP}_{1}$ and $\mathrm{BN}_{1}$ produces over 16 billion tuples and shuffles up to 2.1TB before crashing. The results for SHRED highlight that this is an expensive join, the longest running time for the whole pipeline; however, the succinct dictionary representation allows for a localized join at the first level of the output of $\mathrm{STEP}_{1}$, reducing the join to 10 billion tuples and the shuffling to only 470GB. Thus we avoid carrying around redundant information from the top-level and avoid the outer-join required to properly reconstruct the top-level tuples.

The E2E queries thus exhibit how a succinct representation of dictionaries is vital for executing the whole of the pipeline, with a $7 \mathrm{x}$ advantage for SHRED on the small dataset and scaling to larger datasets when the flattening methods are unable to perform. The queries also highlight how an aggregation pipeline that eventually returns flat output can leverage the succinct representation of dictionaries without the need for reassociating the dictionaries.

\section{RELATED WORK}

Declarative querying against complex objects has been investigated for decades in different contexts, from standalone implementations on top of a functional language, as in Kleisli [58], language-integrated querying approaches such as Links 20 and LINQ 39]. Alternative approaches such as Emma 3 give more fine-grained programming abstractions, integrating into a host language like Scala.

One of the first insights in the field was that queries returning flat output, intermediate subqueries that produce nesting absent in the input can be eliminated. These conservativity results [44,57] were original obtained in the presence of set collections only, but were later extended to multisets, where they are often phrased as a normalization step in processing nested queries 26.

When extending to nested output, a natural approach is to consider the simulation of nested queries on flat representation. This dates to early foundational studies of the nested relational model 56, 22], where it was seen as an extension of conservativity results.

Implementation of this idea in the form of query shredding has been less investigated, but it has been utilized by Cheney et al. 14] in the Links system and Grust et al. 31, 32 in the Ferry system. The thesis of Ultich 54 contains an in-depth overview of Both these systems focus on the generation of SQL queries. Koch et al. 36 provide a shredding algorithm in order to reduce incremental evaluation of nested queries to incremental evaluation of flat queries. They do not deal with scalable evaluation or target any concrete processing platform. However, our symbolic shredding transformation is closely related to the one provided by 36], differing primarily in that the language of 36 supports only queries returning bag type without aggregation.

Query unnesting 26, 35], on the other hand, deals with the programming model mismatch, both for flat queries and nested ones. Fegaras and Maier's unnesting algorithm 26] de-correlates standard NRC queries to support a more efficient bulk implementation, exploiting the richness of the object data model. In the process they introduce an attractive calculus for manipulating and optimizing nested queries. A number of recent applications of nested data models build on this calculus. For example, CleanM 29] uses it as the frontend language for data cleaning and generates Spark code. Similarly, DIQL 25 and MRQL 24 provide embedded DSLs in Scala generating efficient Spark and Flink programs with query unnesting and normalization. These works do not deal with limitations of standard nested representations, nor do they provide support for skew handling. 
Google's large scale analytics systems such as Spanner 8], F1 48, 46, and Dremel 40 support querying against complex objects. Dremel performs evaluation over a "semiflattened" 2] format in order to avoid the space inefficiencies caused by fully flattening data. Skew-resilience and query processing performance are not discussed in 2], which focuses on the impact on storage, while details of the queryprocessing techniques applied in the commercial systems are proprietary. Skew-resilience in parallel processing [10, 37, and methods for efficient identification of heavy keys 45 have been investigated for relational data. But for nested data the only related work we know of targets parallel processing on a low-level parallel language [51, rather than current frameworks like Spark.

\section{CONCLUSION}

Our work takes a step in exploring how components like shredded representations, query unnesting, and skew-resilient processing fit together to support scalable processing of nested collections. Our results show that the platform has promise in automating the challenges that arise for large-scale, distributed processing of nested collections; showing scalable performance for deeper levels of nesting and skewed collections even when state-of-the-art flattening methods are unable to perform at all. In the process we have developed both a micro-benchmark and a benchmark based on a realworld, biomedical use case. A number of components of a full solution still remain to be explored. A crucial issue, and a target of our ongoing work, is cost estimation for these programs, and the application of such estimates to optimization decisions within the compilation framework.

\section{REFERENCES}

[1] I. A. Adzhubei, S. Schmidt, L. Peshkin, V. E. Ramensky, A. Gerasimova, P. Bork, A. S. Kondrashov, and S. R. Sunyaev. A method and server for predicting damaging missense mutations. Nature Methods, 7(4):248-249, 2010.

[2] F. N. Afrati, D. Delorey, M. Pasumansky, and J. D. Ullman. Storing and querying tree-structured records in Dremel. PVLDB, 7(12):1131-1142, 2014.

[3] A. Alexandrov, A. Katsifodimos, G. Krastev, and V. Markl. Implicit Parallelism through Deep Language Embedding. SIGMOD Rec., 45(1):51-58, 2016.

[4] Apache Cassandra, 2020. cassandra.apache.org

[5] Apache CouchDB, 2020. couchdb. apache.org

[6] Apache Hive, 2020. hive.apache.org

[7] M. Armbrust, R. S. Xin, C. Lian, Y. Huai, D. Liu, J. K. Bradley, X. Meng, T. Kaftan, M. J. Franklin, A. Ghodsi, and M. Zaharia. Spark SQL: Relational Data Processing in Spark. In SIGMOD, pages 1383-1394, 2015.

[8] D. F. Bacon, N. Bales, N. Bruno, B. F. Cooper, A. Dickinson, A. Fikes, C. Fraser, A. Gubarev, M. Joshi, E. Kogan, et al. Spanner: Becoming a SQL system. In SIGMOD, 2017.

[9] D. Battré, S. Ewen, F. Hueske, O. Kao, V. Markl, and D. Warneke. Nephele/PACTs: A Programming Model and Execution Framework for Web-Scale Analytical Processing. In Cloud Computing, 2010.
[10] P. Beame, P. Koutris, and D. Suciu. Communication steps for parallel query processing. J. ACM, 64(6):40:1-40:58, 2017.

[11] P. Buneman, S. Naqvi, V. Tannen, and L. Wong. Principles of Programming with Complex Objects and Collection Types. Theoretical Computer Science, 149(1):3-48, 1995.

[12] J. Camacho-Rodríguez, D. Colazzo, I. Manolescu, and J. A. Naranjo. PAXQuery: Parallel Analytical XML Processing. In SIGMOD, 2015.

[13] R. Chaiken, B. Jenkins, P.-Å. Larson, B. Ramsey, D. Shakib, S. Weaver, and J. Zhou. SCOPE: Easy and Efficient Parallel Processing of Massive Data Sets. PVLDB, 1(2):1265-1276, 2008.

[14] J. Cheney, S. Lindley, and P. Wadler. Query Shredding: Efficient Relational Evaluation of Queries over Nested Multisets. In SIGMOD, 2014.

[15] F. Cheng, J. Zhao, and Z. Zhao. Advances in computational approaches for prioritizing driver mutations and significantly mutated genes in cancer genomes. Briefings in Bioinformatics, 17(4):642-656, 082015.

[16] Z. Z. Cheng F, Zhao J. Advances in computational approaches for prioritizing driver mutations and significantly mutated genes in cancer genomes. Briefings in Bioinformatics, 17(4):642-656, 2016.

[17] Citus, 2020. www.citusdata.com.

[18] Cockroach, 2020. https://github.com/cockroachdb/cockroach

[19] Scalable querying of nested data, 2020. github.com/jacmarjorie/trance.

[20] E. Cooper, S. Lindley, P. Wadler, and J. Yallop. Links: Web programming without tiers. In Formal Methods for Components and Objects, 2007.

[21] J. Dean and S. Ghemawat. MapReduce: Simplified Data Processing on Large Clusters. Communications of the ACM, 51(1):107-113, 2008.

[22] J. V. den Bussche. Simulation of the Nested Relational Algebra by the Flat Relational Algebra. Theor. Comput. Sci., 254(1-2):363-377, 2001.

[23] K. Eilbeck, S. E. Lewis, C. J. Mungall, M. Yandell, L. Stein, R. Durbin, and M. Ashburner. The sequence ontology: A tool for the unification of genome annotations. Nature Methods, 6:R44, 2005.

[24] L. Fegaras. An algebra for distributed big data analytics. Journal of Functional Programming, 27, 2017.

[25] L. Fegaras. Compile-time query optimization for big data analytics. Open Journal of Big Data (OJBD), 5(1):35-61, 2019.

[26] L. Fegaras and D. Maier. Optimizing Object Queries Using an Effective Calculus. TODS, 25(4):457-516, 2000.

[27] L. Fegaras and M. H. Noor. Compile-Time Code Generation for Embedded Data-Intensive Query Languages. In BigData Congress, 2018.

[28] Genomic data commons endpoints, 2020. https://docs.gdc.cancer.gov/API.

[29] S. Giannakopoulou, M. Karpathiotakis, B. Gaidioz, and A. Ailamaki. CleanM: An Optimizable Query Language for Unified Scale-out Data Cleaning. PVLDB, 10(11):1466-1477, 2017. 
[30] C. Greenman, P. Stephens, R. Smith, G. L. Dalgliesh, C. Hunter, G. Bignell, H. Davies, J. Teague, A. Butler, C. Stevens, S. Edkins, S. O'Meara, I. Vastrik, E. E. Schmidt, T. Avis, S. Barthorpe, G. Bhamra, G. Buck, B. Choudhury, J. Clements, J. Cole, E. Dicks, S. Forbes, K. Gray, K. Halliday, R. Harrison, K. Hills, J. Hinton, A. Jenkinson, D. Jones, A. Menzies, T. Mironenko, J. Perry, K. Raine, D. Richardson, R. Shepherd, A. Small, C. Tofts, J. Varian, T. Webb, S. West, S. Widaa, A. Yates, D. P. Cahill, D. N. Louis, P. Goldstraw, A. G. Nicholson, F. Brasseur, L. Looijenga, B. L. Weber, Y.-E. Chiew, A. deFazio, M. F. Greaves, A. R. Green, P. Campbell, E. Birney, D. F. Easton, G. Chenevix-Trench, M.-H. Tan, S. K. Khoo, B. T. Teh, S. T. Yuen, S. Y. Leung, R. Wooster, P. A. Futreal, and M. R. Stratton. Patterns of somatic mutation in human cancer genomes. Nature, 446(7132):153-158, 2007.

[31] T. Grust, M. Mayr, J. Rittinger, and T. Schreiber. FERRY: Database-Supported Program Execution. In SIGMOD, 2009.

[32] T. Grust, J. Rittinger, and T. Schreiber. Avalanche-safe LINQ compilation. PVLDB, $3(1-2): 162-172,2010$.

[33] i2b2, 2020. i2b2.org/software/index.html.

[34] International cancer genome consortium, 2020. https://icgc.org/.

[35] W. Kim. On optimizing an SQL-like nested query. TODS, 7(3):443-469, 1982.

[36] C. Koch, D. Lupei, and V. Tannen. Incremental View Maintenance For Collection Programming. In PODS, 2016.

[37] P. Koutris, S. Salihoglu, and D. Suciu. Algorithmic Aspects of Parallel Data Processing. Now Publishers Inc., 2018.

[38] W. McLaren, L. Gil, S. E. Hunt, H. S. Riat, G. R. S. Ritchie, A. Thormann, P. Flicek, and F. Cunningham. The ensembl variant effect predictor. Genome Biology, 17(1):122, 2016.

[39] E. Meijer, B. Beckman, and G. Bierman. LINQ: Reconciling object, relations and XML in the .NET framework. In SIGMOD, 2006.

[40] S. Melnik, A. Gubarev, J. J. Long, G. Romer, S. Shivakumar, M. Tolton, and T. Vassilakis. Dremel: Interactive Analysis of Web-Scale Datasets. PVLDB, 3(1-2):330-339, 2010.

[41] MonetDB, 2020. https://www.monetdb.org/

[42] MongoDB, 2020. www.mongodb.com.

[43] C. Olston, B. Reed, U. Srivastava, R. Kumar, and A. Tomkins. Pig Latin: a not-so-foreign language for data processing. In SIGMOD, 2008.

[44] J. Paredaens and D. Van Gucht. Converting nested algebra expressions into flat algebra expressions. $A C M$ TODS, 17(1):65-93, 1992.

[45] W. Rödiger, S. Idicula, A. Kemper, and T. Neumann. Flow-join: Adaptive skew handling for distributed joins over high-speed networks. In ICDE, 2016.
[46] B. Samwel, J. Cieslewicz, B. Handy, J. Govig, P. Venetis, C. Yang, K. Peters, J. Shute, D. Tenedorio, H. Apte, et al. F1 Query: Declarative Querying at Scale. PVLDB, 11(12):1835-1848, 2018.

[47] K. Sato. An Inside Look at Google BigQuery. White Paper, Google Inc, page 12, 2012.

[48] J. Shute, R. Vingralek, B. Samwel, B. Handy, C. Whipkey, E. Rollins, M. Oancea, K. Littlefield, D. Menestrina, S. Ellner, and et al. F1: A Distributed SQL Database That Scales. PVLDB, 6(11):1068-1079, 2013.

[49] D. Smedley. The biomart community portal: an innovative alternative to large, centralized data repositories. Nucleic Acids Research, 43(W1):W589-W598, 042015.

[50] S. Smemo, J. J. Tena, and M. A. Nóbrega. Obesity-associated variants within fto form long-range functional connections with irx3. Nature, 507(7492):371-375, 2014.

[51] D. Suciu. Implementation and analysis of a parallel collection query language. In $V L D B, 1996$.

[52] D. Szklarczyk, A. Gable, and D. e. a. Lyon. String v11: protein-protein association networks with increased coverage, supporting functional discovery in genome-wide experimental datasets. Nucleic Acids Res, 47(D1):D607-D613, 2019.

[53] Transaction processing performance council, 2020. TPC-H Benchmark. http://www.tpc.org.

[54] A. Ulrich. Query Flattening and the Nested Data Parallelism Paradigm. PhD thesis, University of Tübingen, Germany, 2019.

[55] R. Vaser, S. Adusumalli, S. N. Leng, M. Sikic, and P. Ng. Sift missense predictions for genomes. Nature Protocols, 11(1):1073-1081, 2009.

[56] L. Wong. Querying Nested Collections. PhD thesis, Univ. Pennsylvania, 1994.

[57] L. Wong. Normal forms and conservative extension properties for query languages over collection types. $J$. Comput. Syst. Sci., 52(3):495-505, 1996.

[58] L. Wong. Kleisli, a functional query system. Journal of Functional Programming, 10(1):19-56, 2000.

[59] M. Zaharia, M. Chowdhury, M. J. Franklin, S. Shenker, I. Stoica, et al. Spark: Cluster Computing with Working Sets. HotCloud, 10(10-10):95, 2010.

[60] W. Zhang and S.-L. Wang. A novel method for identifying the potential cancer driver genes based on molecular data integration. Biochemical Genetics, 58(1):16-39, 2020.

[61] Zobra, 2016. https://github.com/zorba-processor/zorba

\section{APPENDIX}

The appendix, beginning on the next page, contains the implementation of the Spark operators, the details of the TPC-H and biomedical benchmark, as well as additional and extended experimental results. 


\begin{tabular}{|c|c|}
\hline Plan Operator & Definition for Dataset API \\
\hline$\sigma_{p(x)}(X)$ & X.filter $(x=>p(x))$ \\
\hline$\pi_{a_{1}, \ldots, a_{k}}(X)$ & $X . \operatorname{select}\left(a_{1}, \ldots, a_{k}\right)$ \\
\hline$\mu^{a_{i}}(X)$ & 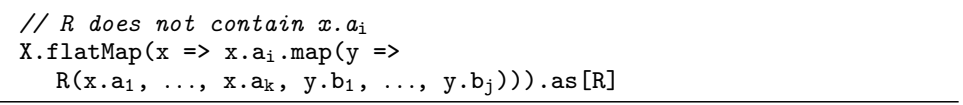 \\
\hline $\boldsymbol{F}^{a}(X)$ & 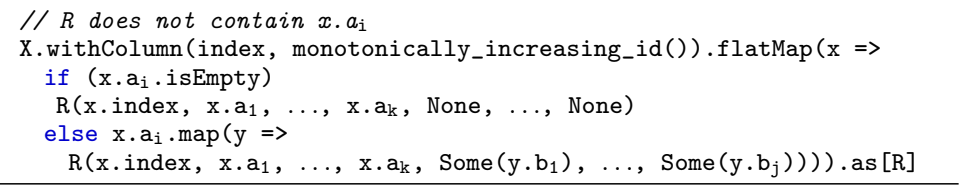 \\
\hline$X \bowtie_{f(x)=g(y)} Y$ & $X \cdot j \circ i n(Y, f===g)$ \\
\hline$X \searrow_{f(x)=g(y)} Y$ & $X . j \circ i n(Y, f===g$, "left_outer") \\
\hline$\Gamma_{+, \operatorname{key}(x)}^{\operatorname{value}(x)}(X)$ & 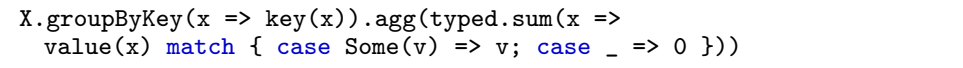 \\
\hline$\Gamma_{\uplus, \operatorname{key}(x)}^{\operatorname{value}(x)}(X)$ & 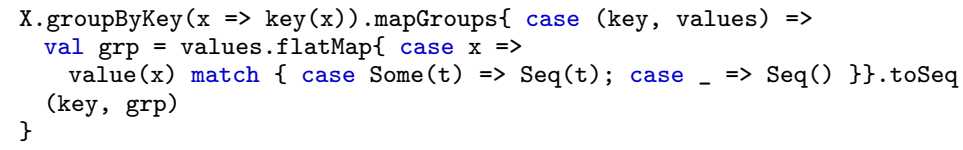 \\
\hline
\end{tabular}

Figure 10: Plan language operators and their semantics for Spark Datasets.

\begin{tabular}{l|l}
\hline Plan Operator & Definition for RDD API \\
\hline$\sigma_{p(x)}(X)$ & X.filter $(\mathrm{x}=>\mathrm{p}(\mathrm{x}))$ \\
\hline$\pi_{a_{1}, \ldots, a_{k}(X)} \mathrm{X} \cdot \mathrm{map}\left(\mathrm{x}=>\mathrm{R}\left(\mathrm{x} \cdot \mathrm{a}_{1}, \ldots, \mathrm{x} \cdot \mathrm{a}_{\mathrm{k}}\right)\right)$
\end{tabular}

Figure 11: Plan language operators and their semantics for Spark RDDs.

\section{A. SPARK IMPLEMENTATION OF THE PLAN OPERATORS}

Figure 10 details the Spark implementations of the plan operators (Section 2.2) applied during code generation (Section 3.2). All operations use Dataset[R], where $\mathrm{R}$ is an arbitrary Scala case class. For outer unnest operation, $\mu^{a}(X)$, we use the function index to create a unique index associated to the top-level input of the unnest operator.

As mentioned in Section 3.2, Spark Datasets are a special instance of RDD that use encoders to avoid the large memory footprint of using RDDs of Scala case classes. Though similar in their underlying data type, the Dataset and RDD APIs are different and thus have different implementations for the language operators. Figure 11 provides the implementation of the operators of the plan language using Spark RDDs. The experiment in Section E.1 highlights the advantages of using Spark Datasets as the underlying type in the code generator. 


\section{B. DETAILED DESCRIPTION OF NESTED TPC-H BENCHMARK}

We detail the nested TPC-H benchmark introduced in our experimental evaluation (Section 6). The queries are designed for a systematic exploration of nested queries within a distributed environment, focusing on a small number of top-level tuples and large inner collections. The queries range from 0 to 4 levels of nesting, organized such that the number of top-level tuples decrease as the level of nesting increases. All queries start with the Lineitem table at level 0, then group across Orders, Customer, Nation, then Region, as the level of nesting increases. Each query has a wide variant where we keep all the attributes, and a narrow variant which follows the grouping with a projection at each level.

For each of the query categories below, we provided the input NRC, the optimal plan produced from the standard compilation route STANDARD and the shredded variant SHRED. The shredded compilation route can produce a plan with or without unshredding (reconstructing the nested object). The shredded compilation route with unshredding will materialized the output of the shredded query and pass it to the unshredding process. Where relevant, we describe the subplan corresponding to the unshredding process and discuss optimizations introduced by the code generator.

\section{B.1 Flat-to-nested}

Here we detail the flat-to-nested queries of the benchmark, which build up nested objects from flat input. For scale factor 100, this organization gives query results with 600 million, 150 million, 15 million, 25, and 5 top-level tuples. Flat-to-nested queries perform the iterative grouping above to the relational inputs, returning a nested output. This starts with the Lineitem table (0 levels), Lineitem grouped by Orders (oparts), oparts grouped by Customers (corders), corders grouped by Nation (ncusts), ncusts grouped by Region (rnations). At the lowest level we keep the partkey and quantity of a Lineitem.

Each of the queries have a narrow and wide variant. At the higher levels the narrow schema keeps only a single attribute, e.g. orderdate for Orders, customername for Customer, etc. The wide variant returns all attributes except at the lowest level, which still just has the partkey and quantity of Lineitem. The ellipses represent the additional fields that may be present based on narrow and wide schemas.

We provide the query with 4 levels, since queries with different levels are merely subsets of this query.

\section{B.1.1 Input NRC Program}

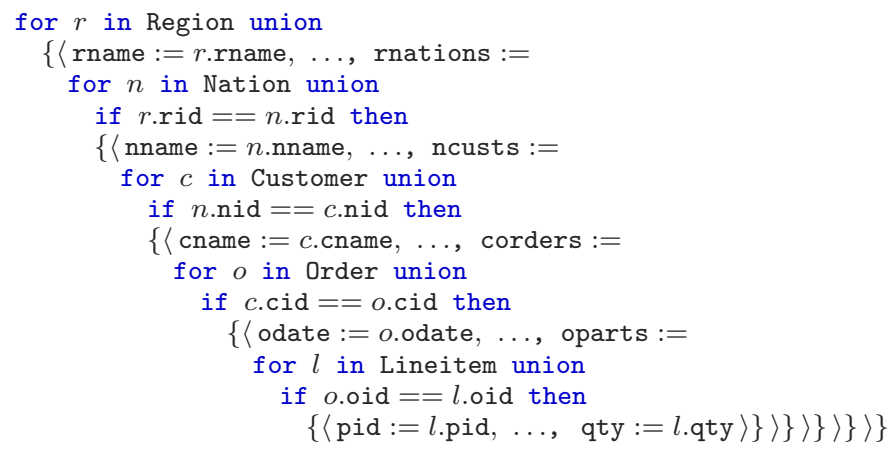

B.1.2 Plan produced by the standard compilation route

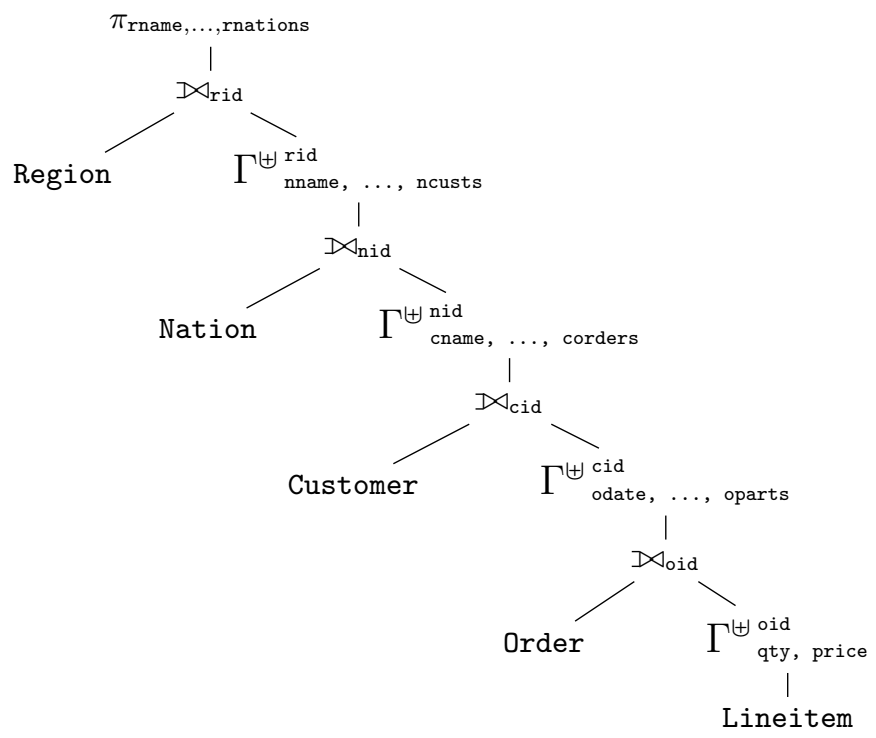

The sequential join-nest operations in the above plan will be merged into cogroups during code generation (Section 3.3). We implement the cogroup in a left-outer fashion, persisting empty bags from the right relation for every matching tuple 
in the left relation. As an example, the join and nest over Order and Lineitem in the above plan will be translated to the following during code generation:

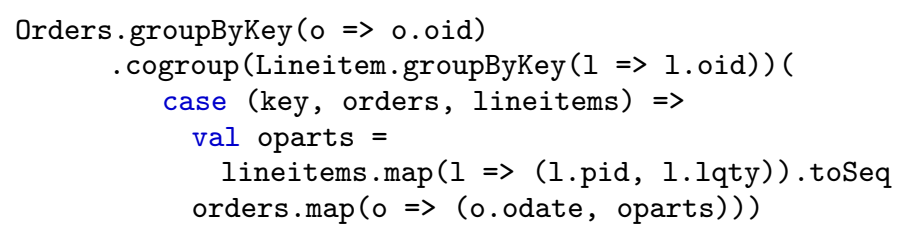

\section{B.1.3 Plan produced by the shredded compilation route}

Below we show the plan produced by the shredded variation of our compilation framework for the evaluation of the shredded query prior to unshredding. The shredded variant produces an additional plan that corresponds to the execution strategy of unshredding; this unshredding plan is identical to the plan produced by the standard compilation route, with each input relation represented as a top-level bag.

$$
\begin{aligned}
& \operatorname{RNCOP}_{\text {Top }}:=\pi_{\text {rname }, \ldots, \text { rnations }:=\text { rid }}(\text { Region }) \\
& \text { rnations }_{\text {Dict }}:=\pi_{\text {label }:=\text { rid,nname,...,ncusts:=nid }}(\text { Nation) } \\
& \text { ncusts }_{\text {Dict }}:=\pi_{\text {label }:=\text { nid,cname, }, \ldots, \text { corders }:=\text { cid }}(\text { Customer }) \\
& \operatorname{corder} \mathbf{S}_{\text {Dict }}:=\pi_{\text {label:=cid,odate, }, . ., \text { oparts:=oid }}(\text { Order }) \\
& \operatorname{oparts}_{\text {Dict }}:=\pi_{\text {label:=oid,pid,qty }}(\text { Lineitem })
\end{aligned}
$$

\section{B.2 Nested-to-nested}

The nested-to-nested queries operate on nested input; these queries take the materialized result of the flat-to-nested queries as input, and perform a join with Part at the lowest level, followed by sumBy pname ${ }_{\text {qty }}$, as in Example 1. The nested-to-nested queries thus produce the same hierarchy and number of top-level tuples as the flat-to-nested queries for all levels of nesting. The ellipses represent the additional fields that may be present in the narrow and wide version of the queries. We provide the query for 4 levels of nesting, all other queries can be derived from this query.

\section{B.2.1 Input NRC Program}

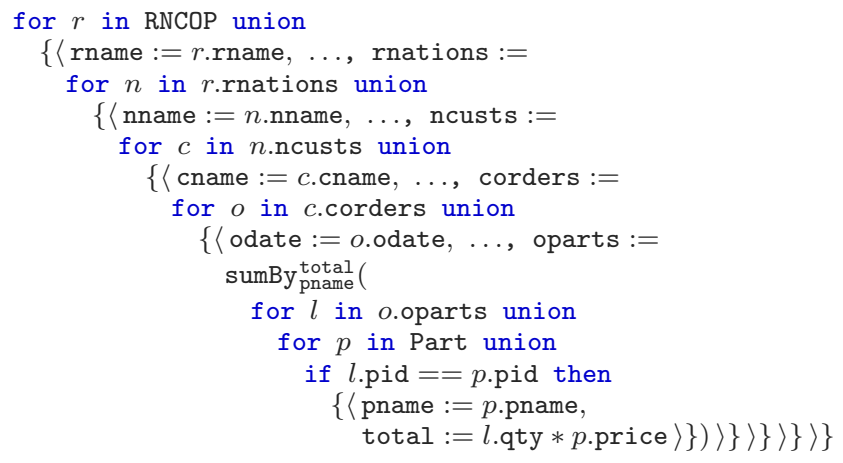




\section{B.2.2 Plan produced by the standard compilation route}

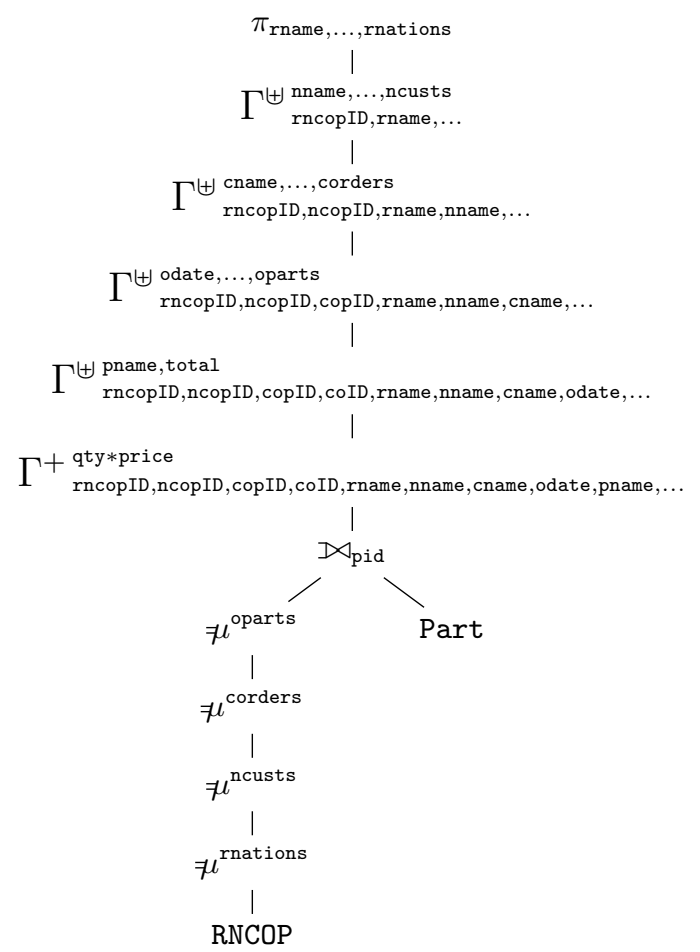

\section{B.2.3 Plan produced by the shredded variant}

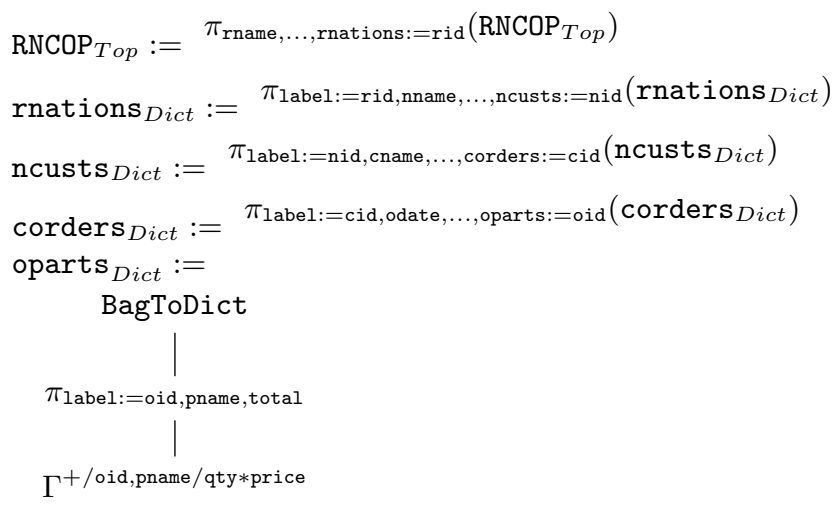

\section{B.3 Nested-to-flat}

The nested-to-flat queries follow the same construction as the nested-to-nested queries, but apply sumBy name $_{\text {qty } \times \text { price }}$ at top-level, where name is one of the top-level attributes; this returns a flat collection persisting only attributes from the outermost level.

The ellipses represent the additional fields that may be present in the narrow and wide version of the queries. We provide the query for 4 levels of nesting, all other queries can be derived from this query. 


\section{B.3.1 Input NRC}

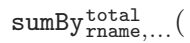

for $r$ in RNCOP union

for $n$ in $n$.rnations union

for $c$ in $n$.ncusts union

for $O$ in c.corders union

for $l$ in o.oparts union

for $p$ in Part union

if $l$.pid $==p$.pid then

$\{\langle$ rname $:=r$.rname,.

total $:=l$.qty $* p$.price $\rangle\}$ )

\section{B.3.2 Plan produced by the standard compilation route}

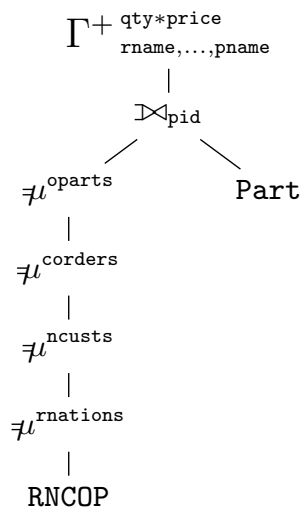

Plan produced by the shredded compilation route.

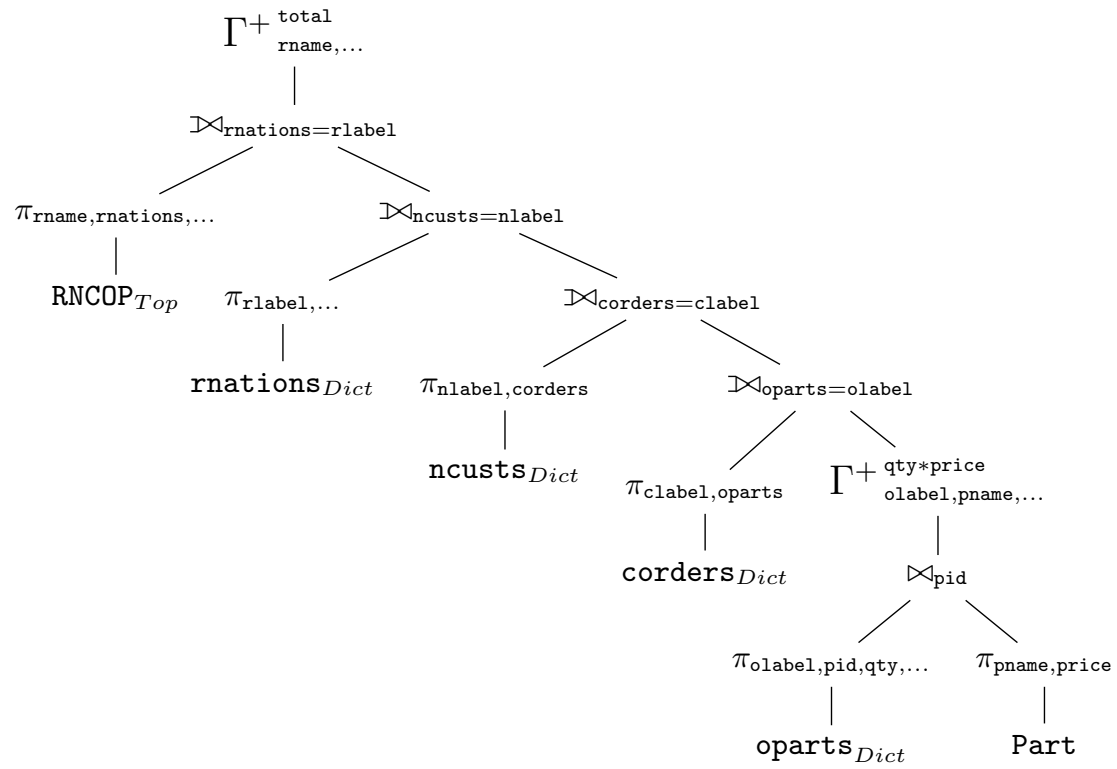




\section{BIOMEDICAL QUERY BENCHMARK}

This section overviews the biomedical benchmark, which was developed in collaboration with a precision medicine start-up. The biomedical benchmark includes an overview of each biomedical data source, queries of the end-to-end (E2E) example from the experiments in Section 6, as well as additional clinical exploration queries and associated performance results. The biomedical benchmark is a collection of NRC queries that perform multiomic analyses, including an end-to-end pipeline $\mathrm{E} 2 \mathrm{E}$ that is based on an analysis that uses several genomic datasets to identify driver genes in cancer 60. Given that cancer progression is determined by the accumulation of mutations and other genomic aberrations within a sample [16], this analysis integrates somatic mutations, copy number information, protein-protein interactions and gene expression data. The benchmark also includes three queries reflecting web-based exploratory analysis that occurs through clinical user interfaces 33. This section continues with details on these datasets and then describes the queries of the analysis and additional queries.

\section{C.1 Inputs}

This section explains the inputs used within the biomedical benchmark. The majority of the datasets are provided from the Genomic Data Commons (GDC) 28, which houses public datasets associated with the International Cancer Genome Consortium (ICGC). The types described below are often truncated for simplicity. The inputs include a two-level nested relation $\mathrm{BN}_{2}(280 \mathrm{~GB})$ 34, 38, a one-level nested relation $\mathrm{BN}_{1}$ (4GB) [52], and five relational inputs - the most notable of which are $\mathrm{BF}_{1}(23 \mathrm{G}), \mathrm{BF}_{2}(34 \mathrm{~GB})$, and $\mathrm{BF}_{3}(5 \mathrm{~KB})$ 34, 23. The implementation of the biomedical benchmark queries can be found in the code repository [19] at https://github.com/jacmarjorie/trance/tree/master/compiler/src/main/scala/ framework/examples/genomic.

\section{C.1.1 $\mathrm{BN}_{2}$ : Occurrences}

An occurrence is a single, somatic mutation belonging to a single sample that has been annotated with candidate gene information. Somatic mutations are cancer-specific mutations that are identified within each sample, and are identified by comparing a sample's cancerous genome to a non-cancerous, reference genome. Note that the term mutation is often used interchangeably with variant. Candidate genes are assigned to mutations based on proximity of a given mutation to a gene on the reference genome. In a naive assignment, a reference gene is a candidate if the mutation lies directly upstream, downstream, or on a gene; however, mutations have been shown to form long-range functional connections with genes 50 and as such candidacy can best be assigned based on a larger flanking region of the genome. With this in mind, the same gene could be considered a candidate gene for multiple mutations within a sample.

Variant annotation is the process that assigns candidate genes to every mutation within each sample. A popular annotation tool is the Variant Effect Predictor (VEP) 38. The Occurrences input is created by associating each simple somatic mutation file (MAF) with nested annotation information from VEP; this returns annotations in JSON format with mutation information at the top-level, a collection of candidate genes for that mutation and corresponding consequence information on the first level, and a collection of additional consequence information on the second level. Each of the mutations within each sample will contain the corresponding nested annotation information.

The tuples in the candidates collection contain attributes that correspond to the impact a mutation has on a gene. The impact attribute is a value from 0 to 1 denoting any known detrimental consequence a mutation has to a candidate gene. sift and poly are additional impact scores that rely on prediction software 55, 1. Given that genes code for proteins and proteins have functional consequences that are attributed disease, these scores reflect the predicted role a mutation has in functional changes to proteins based on alterations to a gene sequence. The consequences for each candidate gene contain qualitative descriptions of mutation impact to a gene sequence based on a standardized set of categorical descriptions from the sequence ontology ( $\mathrm{SO})$ 23].

VEP also takes a distance flag to specify the upstream and downstream range from which to identify gene-based annotations. This flag is used to increase the flanking region of candidate genes associated to each somatic mutation for each sample. From a technical standpoint, increasing the distance will increase the size of candidates and potentially increase the amount of skew. The type of Occurrences is:

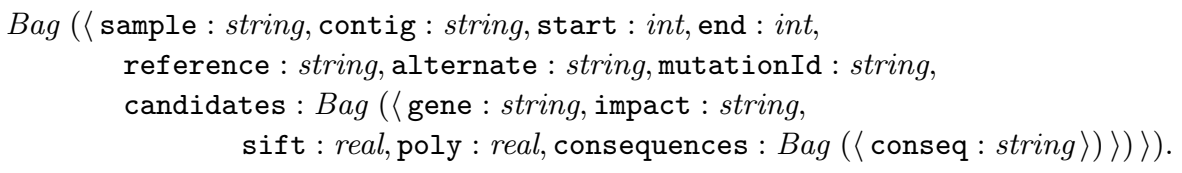

The shredded representation of Occurrences consists of a top-level flat bag Occurrences ${ }^{\mathrm{F}}$ of type:

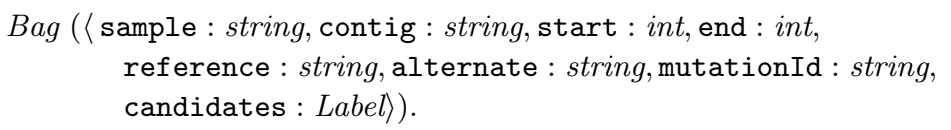


and a dictionary tree Occurrences ${ }^{\mathrm{D}}$ of tuple type

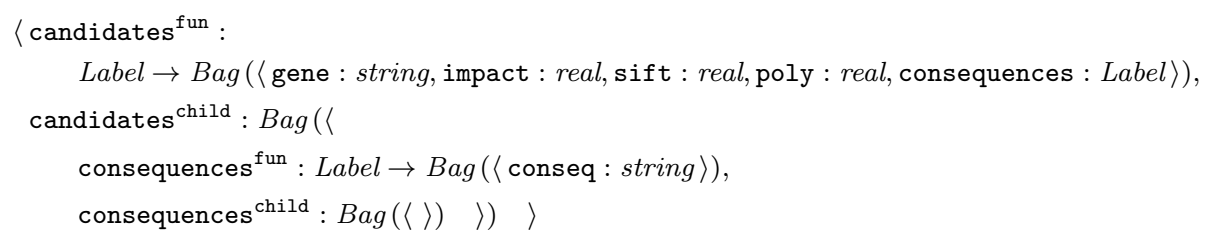

The shredded represented of Occurrences is representated as three datasets in the implementation. The top-level bag is $6 \mathrm{~GB}$, the first-level dictionary is $281 \mathrm{~GB}$, and the third-level dictionary is $35 \mathrm{G}$.

\section{C.1.2 Somatic Mutations and VEP Annotations}

The Occurrences data source described in the above section is based off an endpoint from the ICGC data portal; this resource is built from combining somatic mutation information and variant annotations. Somatic mutations are stored in the GDC as MAF files, which is a flat datadump file that includes a line for every mutation across all samples. The type of the somatic mutation information is:

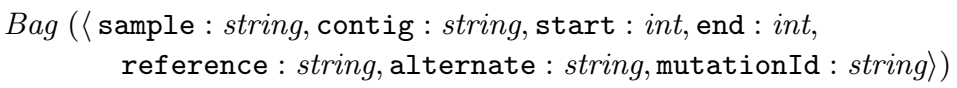

The variant annotations are from the VEP software mentioned above, which returns top-level mutation information and two additional levels of gene and mutational impact information. The overall structure is similar to the occurrences data source, except VEP is returning a unique set of variant annotations that are not associated to a specific sample. The type of the VEP annotation information is:

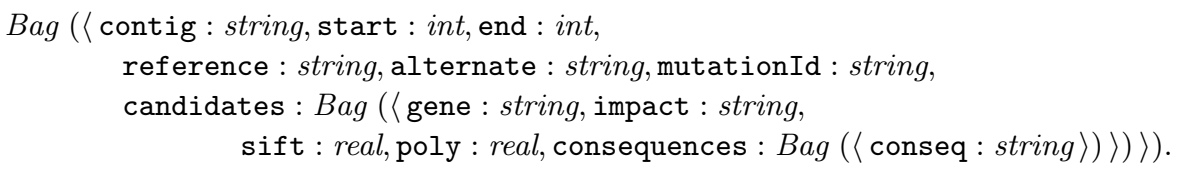

\section{C.1.3 $\mathrm{BF}_{2}$ : Copy Number}

Copy number values correspond to the amplification or deamplification of a gene for each sample, and are also found by comparing against non-cancerous, reference copy number values. The copy number information is provided per gene. The copy number information is reported for each physical sample taken from a patient; this is denoted aliquot. The type of the copy number information is:

$$
\text { Bag ( }\langle\text { aliquot : string, gene : string, cnum : int }\rangle) \text {. }
$$

\section{C.1.4 $\mathrm{BN}_{1}$ : Protein-protein Interactions}

Protein-protein interaction networks describe the relationship between proteins in a network. This Network input is derived from the STRING 52 database. The network is represented with a top-level node tuple and a nested bag of edges, where each edge tuple contains an edge protein and a set of node-edge relationship measurements. The type is:

$$
\text { Bag (〈nodeProtein : string, edges : Bag (〈edgeProtein : string, distance : int }\rangle)\rangle) \text {. }
$$

\section{C.1.5 $\quad \mathrm{BF}_{1}$ : Gene Expression}

Gene expression data is based on RNA sequencing data. Expression measurements are derived by counting the number of transcripts in an aliquot and comparing it to a reference count. The expression measurement is represented as Fragments Per Kilobase of transcript per Million mapped read (FPKM), which is a normalized count. The type is:

$$
\text { Bag ( }\langle\text { aliquot : string, gene : string, fpkm : real }\rangle) \text {. }
$$




\section{C.1.6 Mapping Files}

Sample Metadata. The Samples input maps samples to their aliquots; for the sake of this analysis sample maps to a patient and aliquot associates each biological sample taken from the patient. The type is:

Bag ( $\langle$ sample : string, aliquot : string $\rangle)$.

$\mathbf{B F}_{3}$ : Sequence Ontology. The SOImpact input is a table derived from the sequence ontology 23 that maps a qualitative consequence to a quantitative consequence score (conseq). This is a continuous measurement from 0 to 1 , with larger values representing more detrimental consequences. The type is:

Bag ( $\langle$ conseq : string, value : real $\rangle)$.

Biomart Gene Map. The Biomart input is exported from [49]. It is a map from gene identifiers to protein identifiers. This map is required to associate genes from Occurrences and CopyNumber to proteins that make up Network. The type is:

$$
\text { Bag (〈gene : string, protein : string }\rangle) \text {. }
$$

The inputs described in this section are used in the queries described in the next section.

\section{C.2 E2E: Pipeline Queries}

The queries of the cancer driver gene analysis are an adaptation of the methods from [60]. They work in pipeline fashion to integrate annotated somatic mutation information (Occurrences), copy number variation (CopyNumber), protein-protein network (Network), and gene expression (GeneExpression) data. The idea is to provide an integrated look at the impact cancer has on the underlying biological system. The analysis takes into account the effects a mutation has on a gene, the accumulation of genes with respect to both copy number and expression, and the interaction of genes within the system.

Mutations that play a driving role in cancer often occur at low frequency 30, making cohort analysis across many samples important in their identification. Further, cancer is not just the consequence of a single mutation on a single gene. The interaction between genes in a network, the number of such genes, and their expression levels can provide a more thorough look at cancer progression 15. The queries below define the analysis. The queries work in pipeline fashion where the materialized output from one query is used as input to a query later on in the pipeline.

The pipeline starts with the integration of mutation and copy number variation to produce a set of hybrid-scores for each sample. The hybrid-scores are then combined with network interactions to determine effect-scores. The effect-scores are further combined with gene expression information to determine the connection scores for each sample. The queries conclude by combining the connection scores across all samples, returning connectivity scores for each gene. The genes with the highest connectivity scores are considered drivers. 


\section{C.2.1 STEP 1 : Hybrid scores}

The hybrid score query is the first step in the pipeline. A hybrid-score is calculated for each candidate gene within a sample by combining mutation impact and copy number information for that sample, thus providing a score that corresponds to the likelihood that the gene is a driver. The output of this step remains grouped by sample in order to continue integrating sample-specific genomic datasets that can further contribute to our understanding of driver genes in cancer in the steps below.

The query below describes the process of creating hybrid scores based on the 0ccurrences input. The main difference between this version and the running example is that Samples provides a map between sample and aliquot used to join CopyNumber, and the hybrid score is determined for every aliquot. In addition, conditionals are used to assign qualitative scores based on the human-interpretable level of impact (impact).

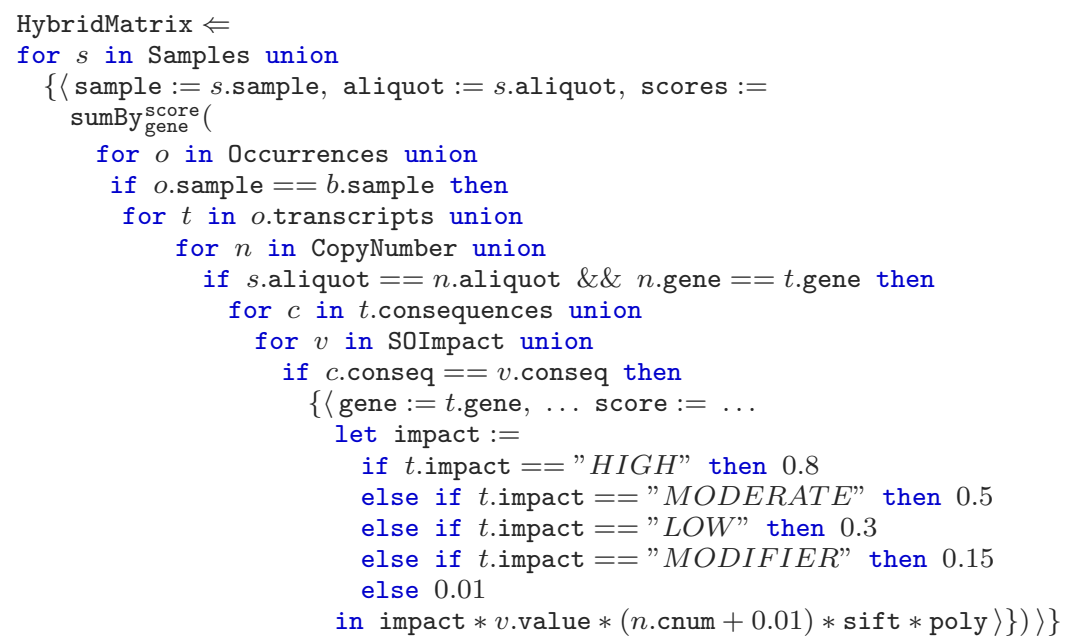

The output type of this query is:

Bag (〈sample : string, aliquot : string, scores : Bag (〈gene : string, score : real $\rangle)\rangle)$.

The plan produced by our standard compilation route is:

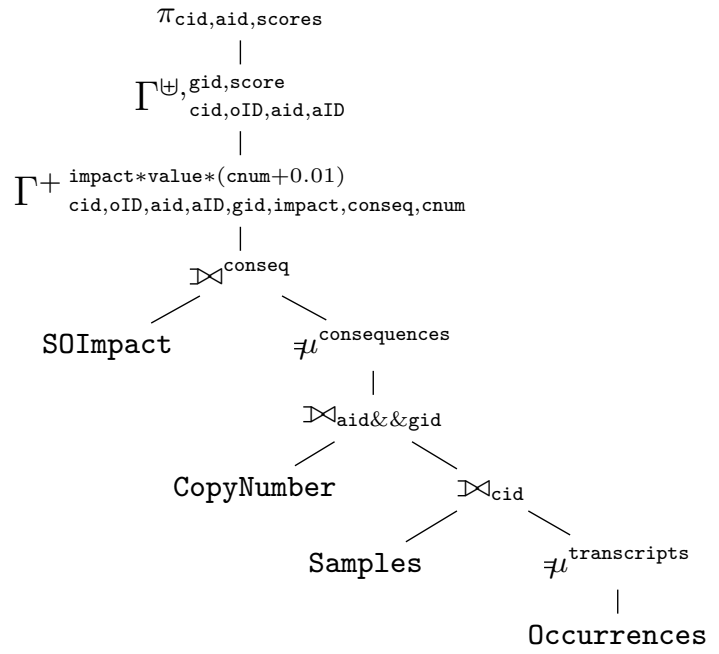




\section{C.2.2 $\mathrm{STEP}_{2}$ : By Sample Network}

The second step in the pipeline aggregates on an individual's sample network, based on the hybrid scores. The goal is to associate each gene in the nested edges collection of Network with the corresponding hybrid scores for a sample. For each gene in this collection, the product of the hybrid score and the relationship measurement for that edge (distance) are summed for each node in the network for each sample.

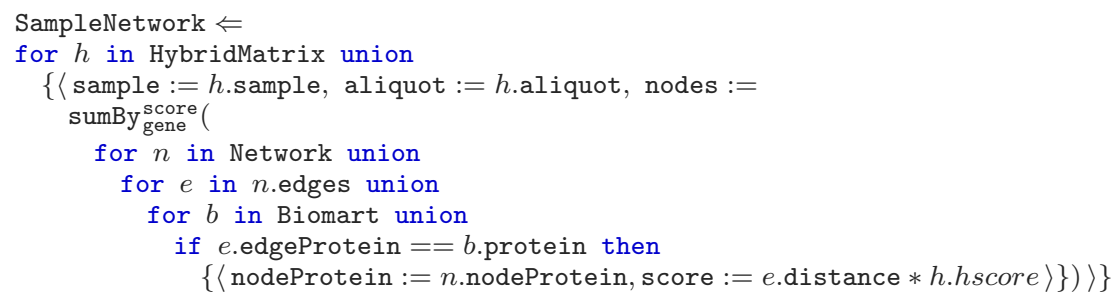

The output type of this query is:

Bag (〈sample : string, aliquot : string, nodes : Bag (〈nodeProtein : string, score : real $\rangle)\rangle)$.

\section{C.2.3 $\mathrm{STEP}_{3}:$ Effect scores}

The effect scores are calculated using the materialized output of the previous two steps, denoted SampleNetwork and HybridMatrix. The nested nodes collection of SampleNetwork contains the sum of the combined gene interaction and hybrid score across the edges collection for each node gene in the top-level tuples of Network. These values are then combined with the hybrid scores for each node gene to produce the effect matrix.

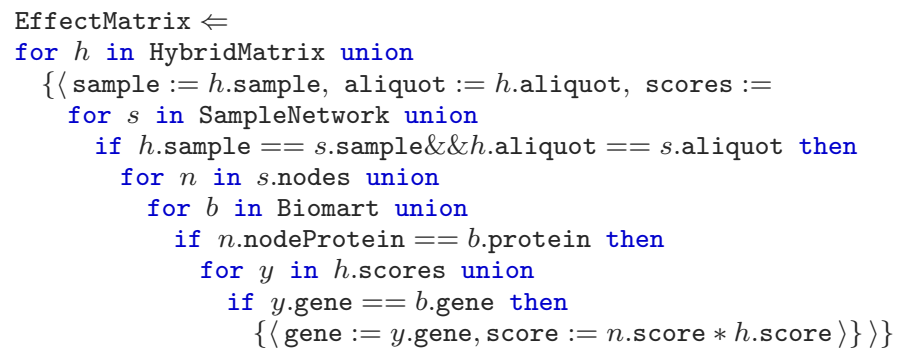

The output type is:

$$
\text { Bag (〈sample : string, aliquot : string, scores : Bag (〈gene : string, score : real }\rangle)\rangle) \text {. }
$$

Step $_{4}$ : Connection scores. Connection scores are determined by combining the effect scores and gene expression data. Gene expression data uses the normalized count (FPKM) measurement discussed in the input section above.

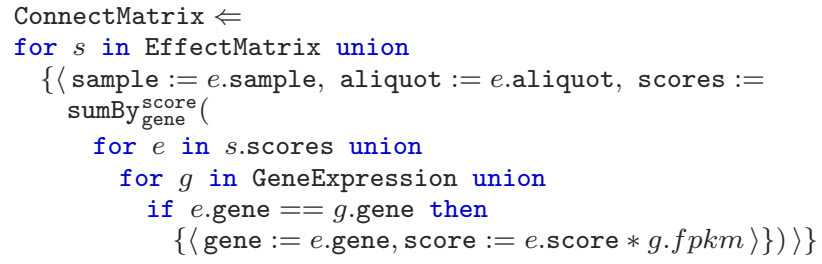

The output type is:

$$
\text { Bag (〈sample : string, aliquot : string, scores : Bag (〈gene : string, score : real }\rangle)\rangle) \text {. }
$$




\section{C.2.4 STEP 5 : Gene connectivity}

The gene connectivity sums up connection scores for each gene across all samples. The genes with the highest connection scores are taken to be drivers.

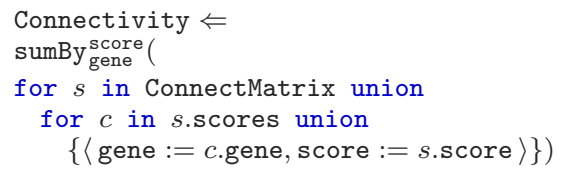

The output type is:

$$
\text { Bag (〈gene : string, score : real }\rangle) \text {. }
$$

\section{C.3 Clinical exploration queries}

This section provides an overview of the clinical exploration queries in the biomedical benchmark. The clinical exploration queries reflect requests a clinician may make from a user-interface; for example, an electronic health record system that provides access to Occurrences and CopyNumber. The queries contain a combination of restructuring, nested joins, and aggregation. The queries are nested-to-nested and each query applys an additional operation on the next. $\mathrm{C}_{1}$ groups $\mathrm{BN}_{2}$ to return a three-level nested output. $\mathrm{C}_{2}$ joins $\mathrm{BF}_{2}$ at level 1 of $\mathrm{BN}_{2}$ then groups as in $\mathrm{C}_{1}$. $\mathrm{C}_{3}$ proceeds the same way and aggregates the result of the join prior to grouping.

\section{C.3.1 $\mathrm{C}_{1}$ : Group occurrences by sample}

This query groups occurrences by sample producing a bag of nested mutation information for each sample. The query also associates a quantitative value to the consequences at the lowest level in the process. The output has four levels of nesting.

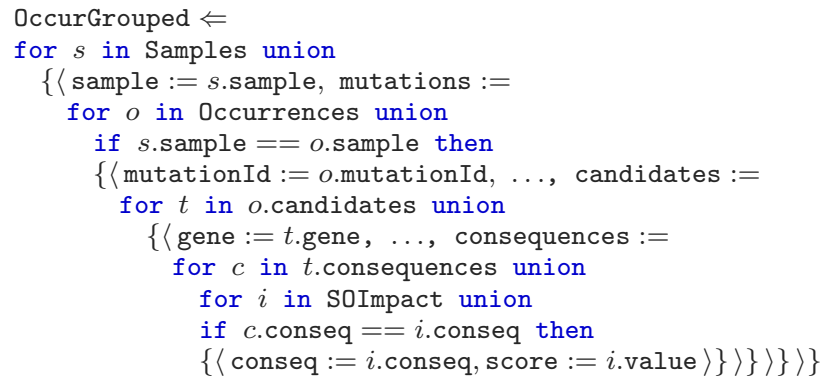

\section{C.3.2 $\mathrm{C}_{2}$ : Integrate copy number and occurrences, group by sample}

This query is similar to above, but joins copy number data on the second level - per each gene - while constructing mutation groups per sample.

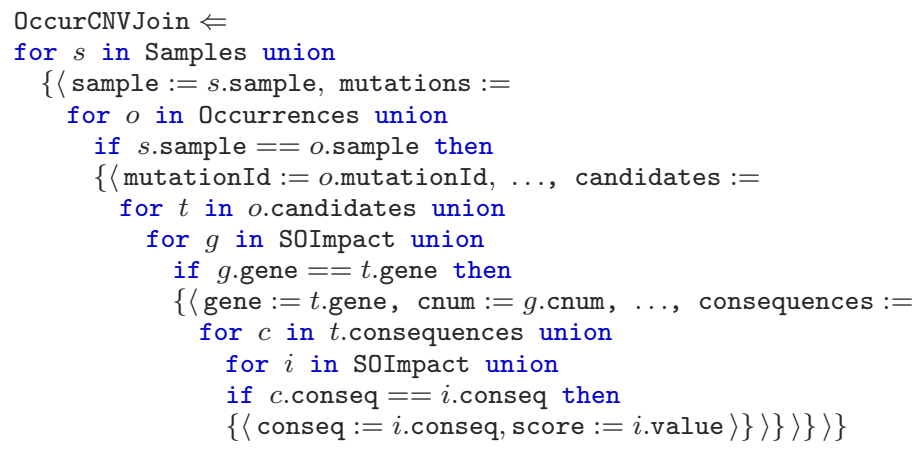




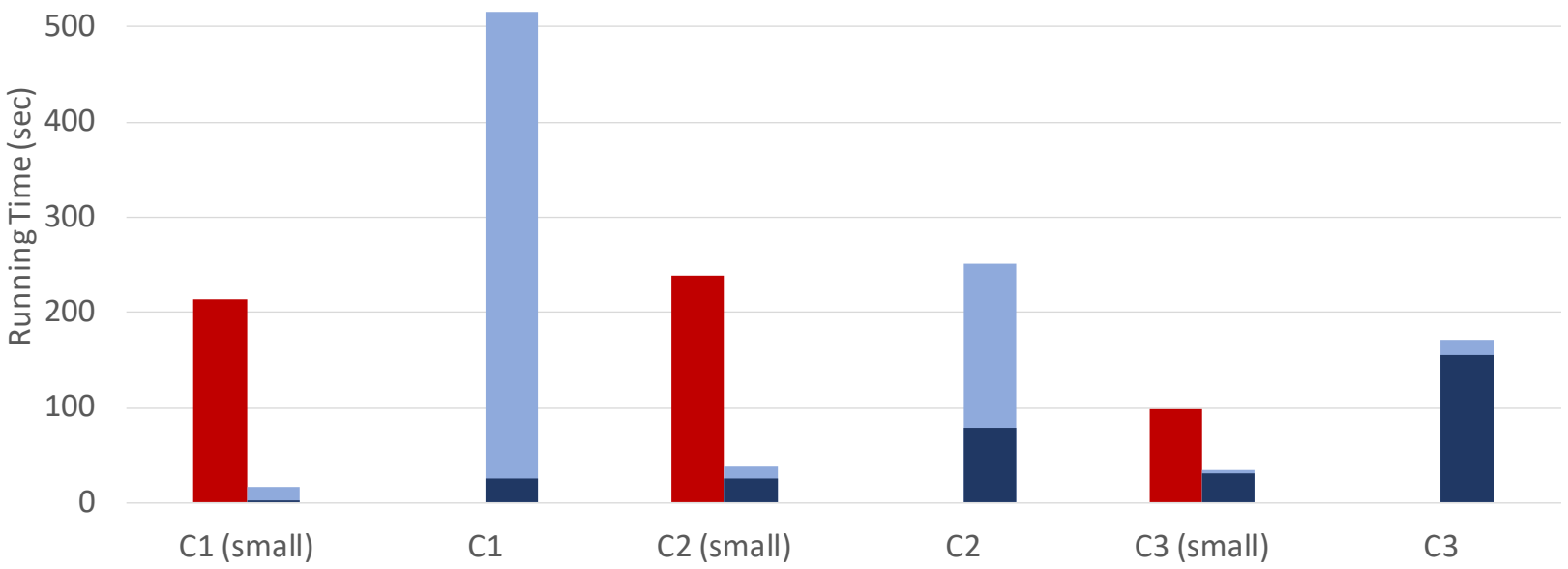

Figure 12: Results for the clinical exploration queries.

\section{C.3.3 $\mathrm{C}_{3}$ : Aggregate copy number and occurrences, group by sample}

This query groups by sample, joins copy number at the second level, joins quantitative consequence values at the third level, and aggregates the product of copy number and consequence score for each gene.

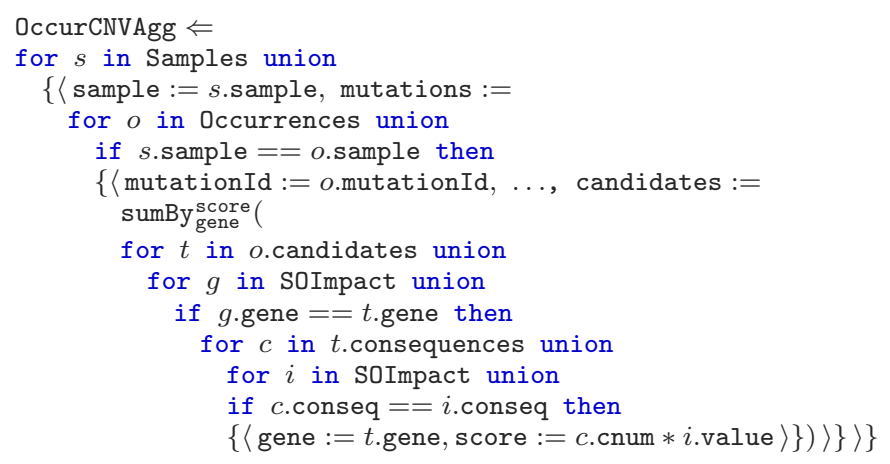

\section{C.3.4 Performance}

The clinical queries extend the biomedical benchmark to explore how queries perform that are not necessarily part of a whole analysis pipeline. These queries are indicative of a process that would return all data to display in a user-interface, which is synonymous to fewer projections in the output.

The clinical exploration queries were evaluated using varying sizes of the Occurrences input; one small collection based on a $168 \mathrm{M}$ mutation file (identified by small) and a large collection of $42 \mathrm{G}$ annotated mutations. Note this is a smaller dataset that is used in the E2E. Figure 12 displays these results. STANDARD was unable to run to completion for the larger input for all queries, overloading the available memory on the system each time. The shredded variant of our compilation was able to complete for all queries exhibiting resilience by distributing the large inner collections. 


\section{NOTE ABOUT SUCCINCT REPRESENTATION AND SHARING}

We use the somatic mutations and VEP annotations described in Section C.1 to explore the benefits of sharing. This microexperiment uses one MAF file from the breast cancer dataset containing 120988 tuples, and the associated VEP annotations for the unique set of all mutations (58121 tuples). When the mutations are joined with the annotation table in the standard compilation route, the result contains 5170132 nested transcript consequence tuples. In the shredded compilation route, the mutations are joined with the top-level annotation dictionary, and first-level dictionary of the output is just the first-level transcript consequences dictionary from the input (3777092 tuples). In comparison to the standard compilation route, the dictionary representation has reduced the total size of the transcript tuples by over 1 million tuples. These results are based off a small subset of the data. Since many of the samples will share mutations specific to cancer, the benefits of sharing will increase as the number of samples increases.

\section{E. ADDITIONAL AND AUGMENTED EXPERIMENTAL RESULTS}

This section contains additional experimental results, along with extended experimental results and some of the experimental plots in the paper augmented with memory information.

All results are for 100GB of non-skewed TPC-H data (scale factor 100, skew factor 0). We use the flat-to-nested and nestedto-nested queries to compare the performance of the standard and shredded variants of our framework using both RDDs and Datasets. As in the body of the paper, we use STANDARD (standard compilation route), ShrED (shredded variant without unshredding), and UNSHRED (shredded variant with unshredding of the final output) to represent runs from our framework. We also explore the effects of introducing database-style optimizations on the plans of the standard compilation route. The results highlight advantages of using Datasets over RDDs in code generation, particularly for nested collections. The results also show how introducing database-style optimizations can significantly improve performance of the standard compilation route, generating programs similar to programs that have been optimized by hand.

\section{E.1 Spark RDDs vs Spark Datasets}

The flat-to-nested and nested-to-nested queries are used to compare the performance of STANDARD (standard compilation route), SHRED (shredded compilation route without unshredding of output), and UNSHRED (shredded compilation with unshredding) using RDDs and Datasets. Figure 13 displays the results for the flat-to-nested queries with $13 \mathrm{a}$ and without projections (13b). With projections, the results show that all strategies exhibit similar performance up to three levels of nesting. The strategies diverge at four levels of nesting where UNSHRED and STANDARD with RDDs have a spike in total run time. The results without projections follow a similar trend, with strategies diverging earlier at lower levels of nesting. Without projections, UNSHRED with RDDs shows increasingly worse performance starting at two levels of nesting. SHRED with RDDs and SHRED with Datasets have similar performance.

As stated in Section B.1 the plan produced with unshredding in the shredded compilation and the plan for the standard compilation are identical; thus, the difference in performance is attributed to code generation for the unshredding procedure. Both methods use a series of cogroups to build up a nested set; however, unshredding requires additional map operations and intermediate object creation that are required for reconstructing nested objects from dictionaries. Case classes that lack binary encoders require a significant amount of time and space to create and store, which is a cost that only increases with the levels of nesting. UNSHRED with RDDs grows exponentially as the number of nesting increases, bringing along the previous level with each level of nesting. This is also a problem for STANDARD, which sees worse performance with increasing levels of nesting but grows at a slower rate due to less object creation.

To consider what this means from a code generation perspective, compare the project operator of Figure 10 to the project operator in Figure 11. The RDD API maps over a relation and creates a new case class (R), whereas the Dataset API avoids this map and uses a select operator that accepts a series of attribute names. By explicitly stating the attributes, the Dataset API has delayed an explicit map operation allowing for further performance benefits from the Spark optimizer. Further, when the time comes to construct R objects, the Dataset API leverages the binary format for a much smaller memory footprint.

Figure 14 further highlights the benefits of Datasets with nested-to-nested queries. Both with and without projections, the difference between SHRED with RDDs and SHRED with Datasets shows a $2 \times$ performance improvement of the explicit statement of attributes within the Dataset API, and STANDARD has decreased performance with RDDs. While the unnest operators used in the code generators both use flat-map operations, the Dataset API maintains a low-memory footprint for the newly created objects (.as [R] in the code generator).

These results show that code generation with Datasets has minimized overhead in object creation and gains further improvements from passing meta-information to the Spark optimizer. Beyond the application to Spark, these results should be useful for further implementations of automated nested query processing on distributed systems. 


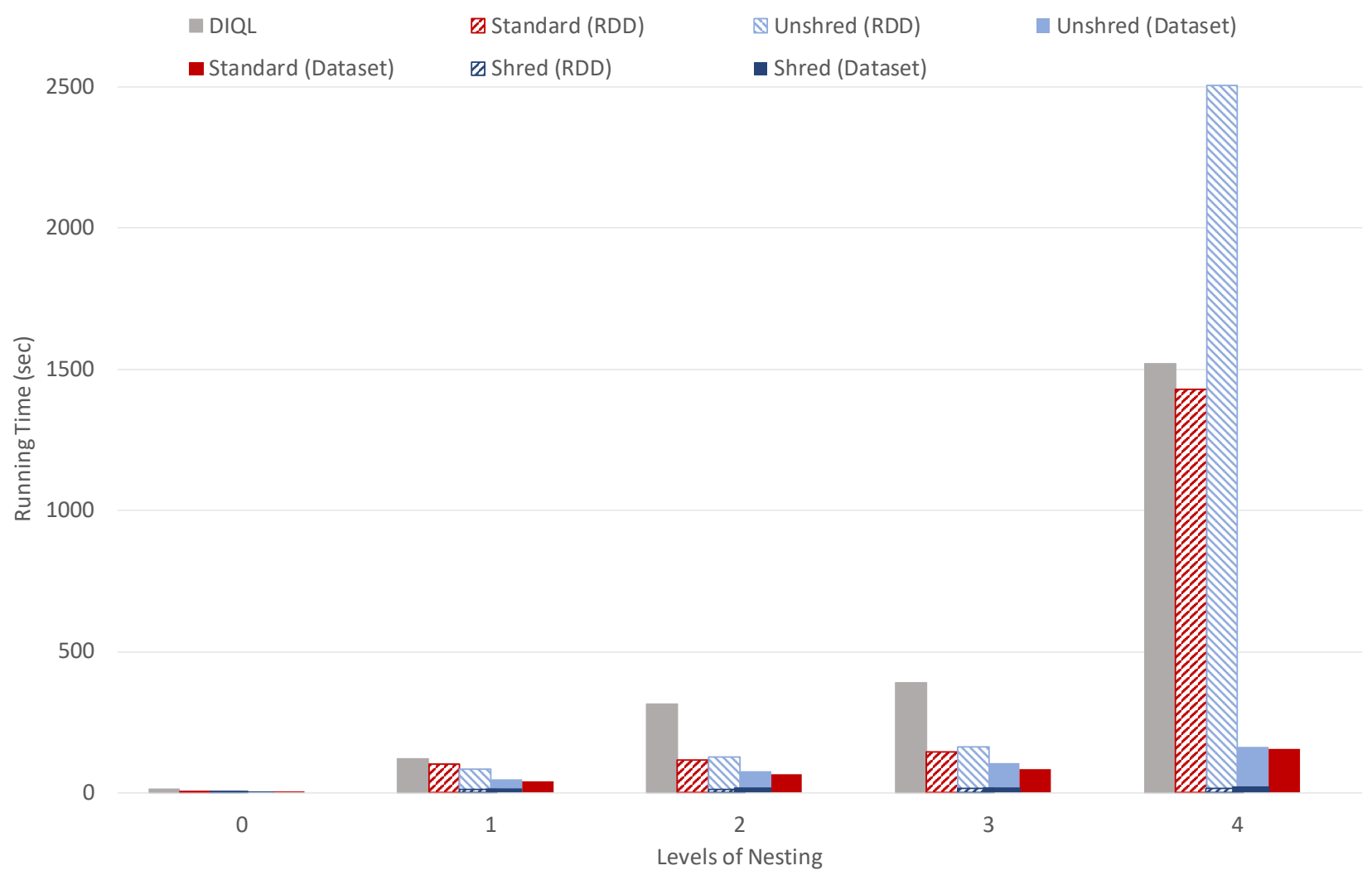

(a) Narrow schema

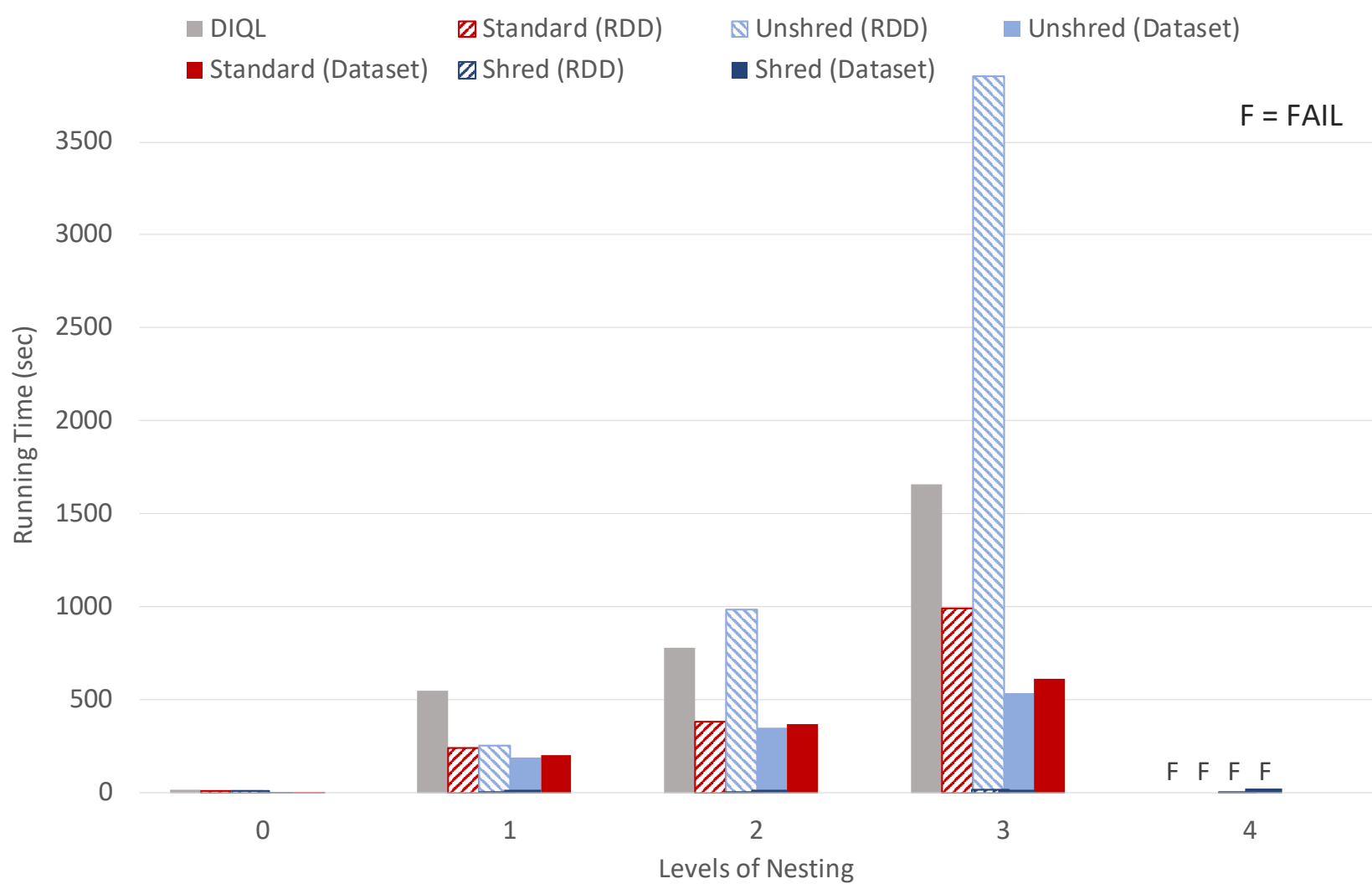

(b) Wide schema

Figure 13: Performance comparison of RDDs and Datasets for the flat-to-nested benchmarked queries, including additional competitor DIQL that uses an RDD implementation. 


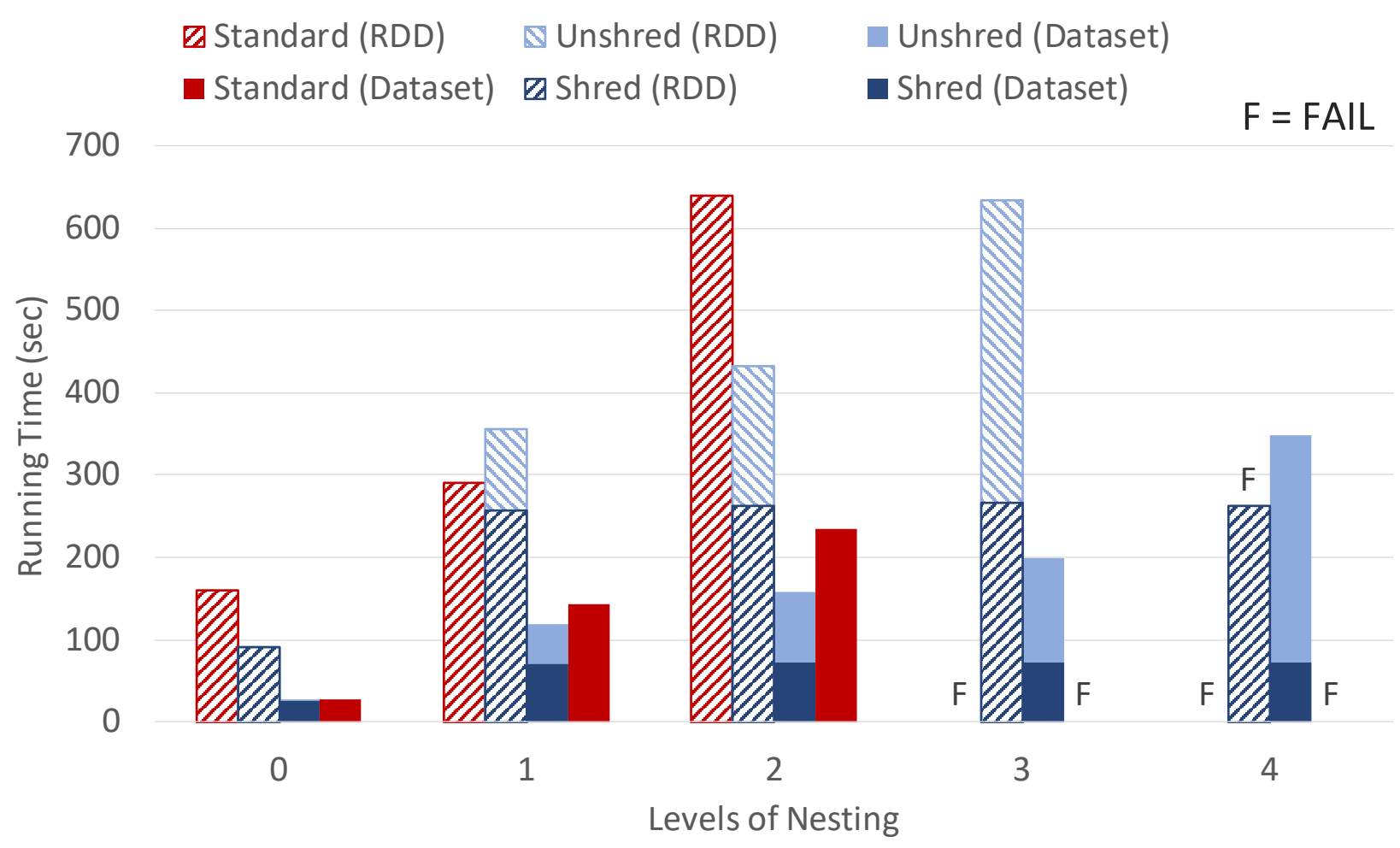

(a) Narrow schema

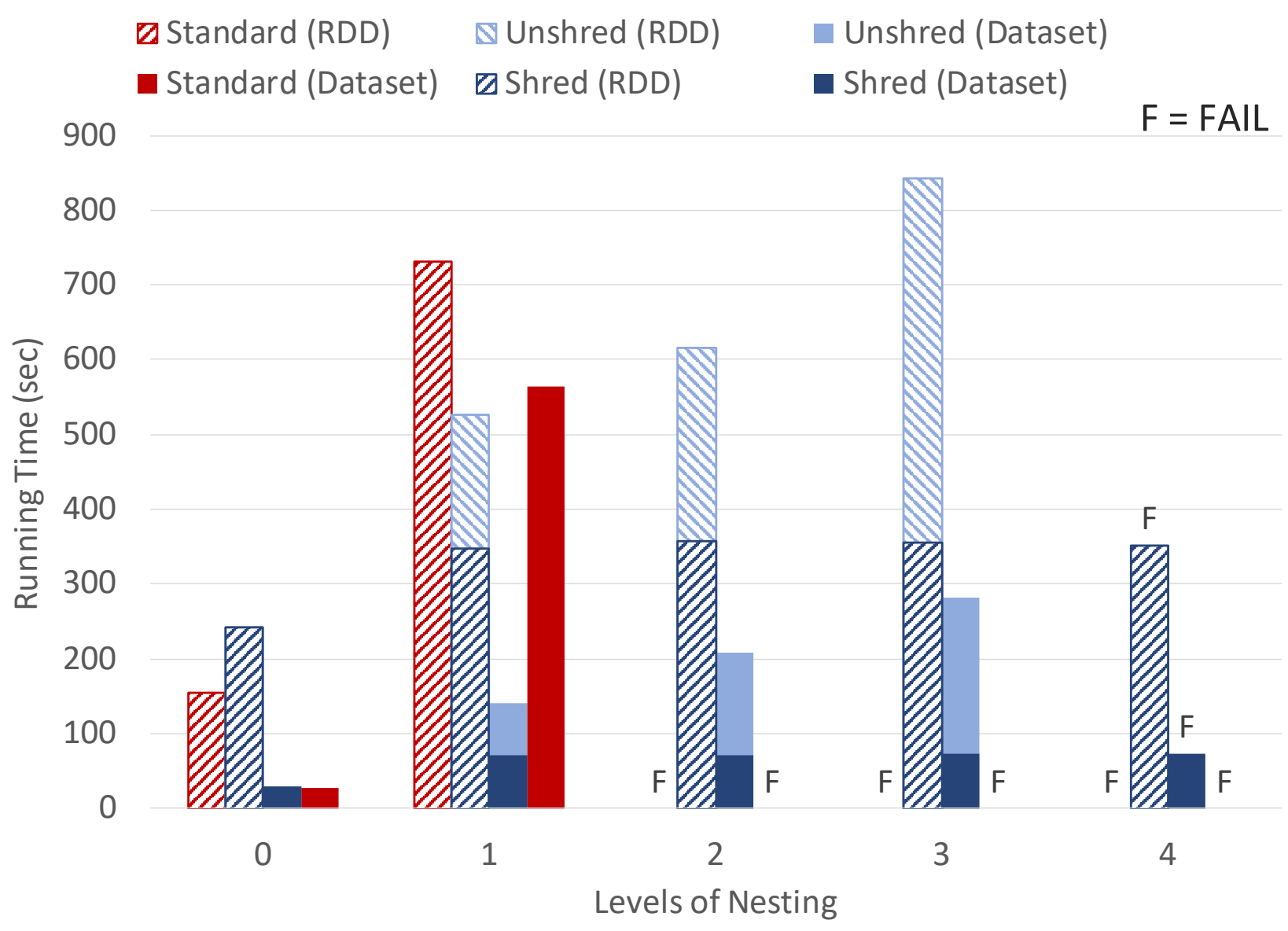

(b) Wide schema

Figure 14: Performance comparison of RDDs verse Datasets for the nested-to-nested benchmarked queries. 


\section{E.2 Experiments Comparing With Additional Competitors}

We used the TPC-H benchmark to compare to a wide array of external competitors: an implementation via encoding in SparkSQL [7] Citus, a distributed version of Postgres [17]; MongoDB [42], and the recently-developed nested relational engine DIQL 25. We include the results for SparkSQL since it outperformed all the other competitors. This section shows the extended results, including all competitors, for the flat-to-nested, nested-to-nested, and nested-to-flat queries. The source code for each of the queries is available in the github.

Evaluation strategies and competitors. We explored several potential competitors for use in the comparison. The following competitors were able to perform at least one of the TPC-H benchmark queries, and thus are represented in the subsequent results.

- SparkSQL:

The SparkSQL queries were manually written based on two restrictions. First, SparkSQL does not support explode (i.e., UNNEST) operations in the SELECT clause, requiring the operator to be kept with the source relation which forces flattening for queries that take nested input. Second, an (outer) join cannot follow an explode statement; this means the query must be written in the following form:

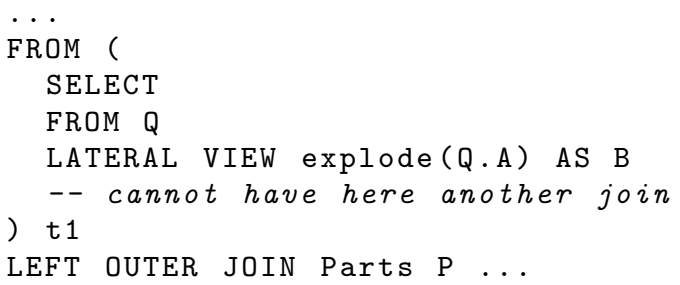

- $D I Q L$ :

The syntax of DIQL fully supports all the queries in the TPC-H benchmark; however, this is an experimental system and we uncovered bugs during this process. With this in mind, we provide the results for the flat-to-nested queries only. The DIQL Spark API has slightly different system requirements and we were only able to compile and run the queries with Spark 2.4.3 and Scala 2.11.

- Postgres+Citus:

We use a distributed version of Postgres (Citus) as the representative relational database engine. We use a coordinator Postgres instance with five workers, exactly like the Spark. We cached inputs and parallelized processes as much as possible with the following:

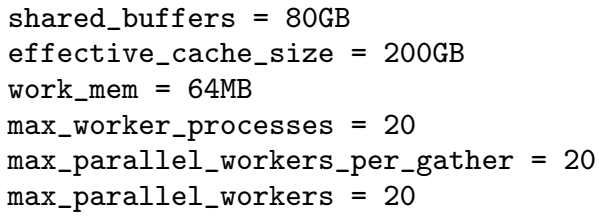

However, the results using default values had better performance. The Citus queries were manually written, using both arrays and JSON with and without caching inputs. We report the runtimes of the array based queries, without caching inputs, and default worker configurations since this continuously outperformed the others.

All Citus queries are based on several caveats. First, Citus does not support nested subqueries in the target of a SELECT, failing with could not run distributed query with subquery outside the FROM, WHERE and HAVING clauses. Second, queries can be rewritten using GROUP BY and ARRAY_AGG, but joins between relations partitioned on different columns - known as complex joins in Citus terminology - are not supported; this fails with complex joins are only supported when all distributed tables are co-located and joined on their distribution columns. Outer joins can be done in a binary fashion with one table being a common table expression (CTE). For instance, the following query where t1 and t2 are partitioned on the join key but not on the join key for $\mathrm{t} 3$ :

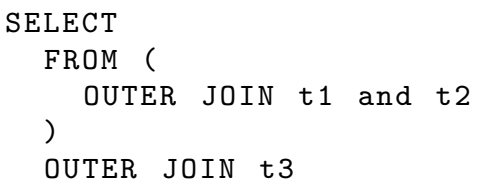

The result of the subquery ( $\mathrm{t} 1$ join $\mathrm{t} 2$ ) will be collected entirely at the master and then partitioned to workers according to the next join key. This is obviously inefficient and has restrictions logged in Citus as:

DETAIL: Citus restricts the size of intermediate results of complex subqueries and CTEs to avoid accidentally pulling large result sets into once place. 
Third, left outer joins between tables partitioned on different keys are not yet supported/https://github.com/citusdata/ citus/issues/2321. Finally, to avoid pulling data back to master and enable outer joins between relations partitioned on different keys, we manually created execution plans where at each step we (outer) join two relations partitioned on the same key and write the result back into a distributed materialized view partitioned on the next join key in sequence. That means we had to materialize the entire flattened nested object to get it repartitioned by partkey before joining with Part. Each nested-to-flat requires 2 queries, while each nested-to-nested has one extra query for the final regrouping.

- MongoDB:

We use MongoDB with one master and five workers, as in the Spark and Citus setup. The queries were hand-written based on the following restrictions. Only one collection can be sharded when performing lookups (joins), the inner one must be local. The only join strategy is to iterate (in parallel) over the outer collection and do lookups on the inner collection, which is located on one machine; thus, this is a bottleneck. We find that MongoDB has good performance with selective filters over a single collection, not designed for queries over multiple collections or even single-collection queries that return many documents. Nested collections formed using the \$push accumulator are currently capped at 100MB; pipelines using more than 100MB will fail.

Additional competitors explored. The following systems were also explored, but were unable to support the queries of the benchmark.

- Rumble:

Rumble transforms JSONiq to Spark and supports local and distributed execution. We discovered problems running even toy examples doing data denormalization. Initially, outer joins were not supported: https://github.com/RumbleDB/ rumble/issues $/ 760$. We reported this and it was fixed, but now outer joins with distributed collections are transformed into Cartesian products ${ }^{1}$ In general, the Rumble JSONiq language is not providing several of the operations available in Spark (e.g., caching, schema handling) are not available through their JSONiq language.

- Zorba:

Zorba (JSONiq) has no support for distributed execution, and has not been maintained in the past 4 years 61.

- MonetDB:

MonetDB [41] has no support for array type and array operations. The system does support JSON operations over strings, but there is no easy way to transform tables to JSON objects; manually creating JSON strings throws errors.

- Cockroach:

CockroachDB does not support nested arrays or ordering by arrays [18.

- VoltDB:

VoltDB does not have support for arrays. There is support for JSON, but is is up to the application to perform the conversion from an in-memory structure to the textual representation. In addition, there is a size limit for JSON values. The VARCHAR columns used to store JSON values are limited to one megabyte (1048576 bytes). JSON support allows for augmentation of the existing relational model with VoltDB; however, it is not intended or appropriate as a replacement for pure blob-oriented document stores.

- YugabyteDB:

YugabyteDB seemed like a good candidate as it supports distributed execution and much of SQL, but the performance was too poor to explore further. For example, the following query took four minutes with 18760 orders tuples and 2500 user tuples:

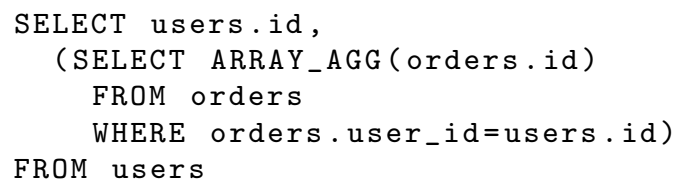

Flat-to-nested for non-skewed data. Figure 15a displays the results for MongoDB, Postgres Citus, DIQL, SparkSQL, StAndARD, Shred, and Unshred for the narrow flat-to-nested queries of the TPC-H benchmark. Given the performance for all systems is worse for wide tuples, we did not explore the performance of MongoDB and Citus for the wide variants. Figure 15b displays the results for DIQL, SparkSQL, StAndARD, Shred, and Unshred for the wide flat-to-nested queries.

Nested-to-nested for non-skewed data. Figure 16 displays the results for MongoDB, Postgres Citus, SparkSQL, StANDARD, SHRED, and UnSHRED for the narrow nested-to-nested queries of the TPC-H benchmark. Given the performance for all systems is worse for wide tuples, we did not explore the performance of MongoDB and Citus for the wide variants; thus, the wide variants for SparkSQL, STANDARD, SHRED, and Unshred can be found in the main body of the paper.

\footnotetext{
${ }^{1}$ Accessed 10 September 2020.
} 


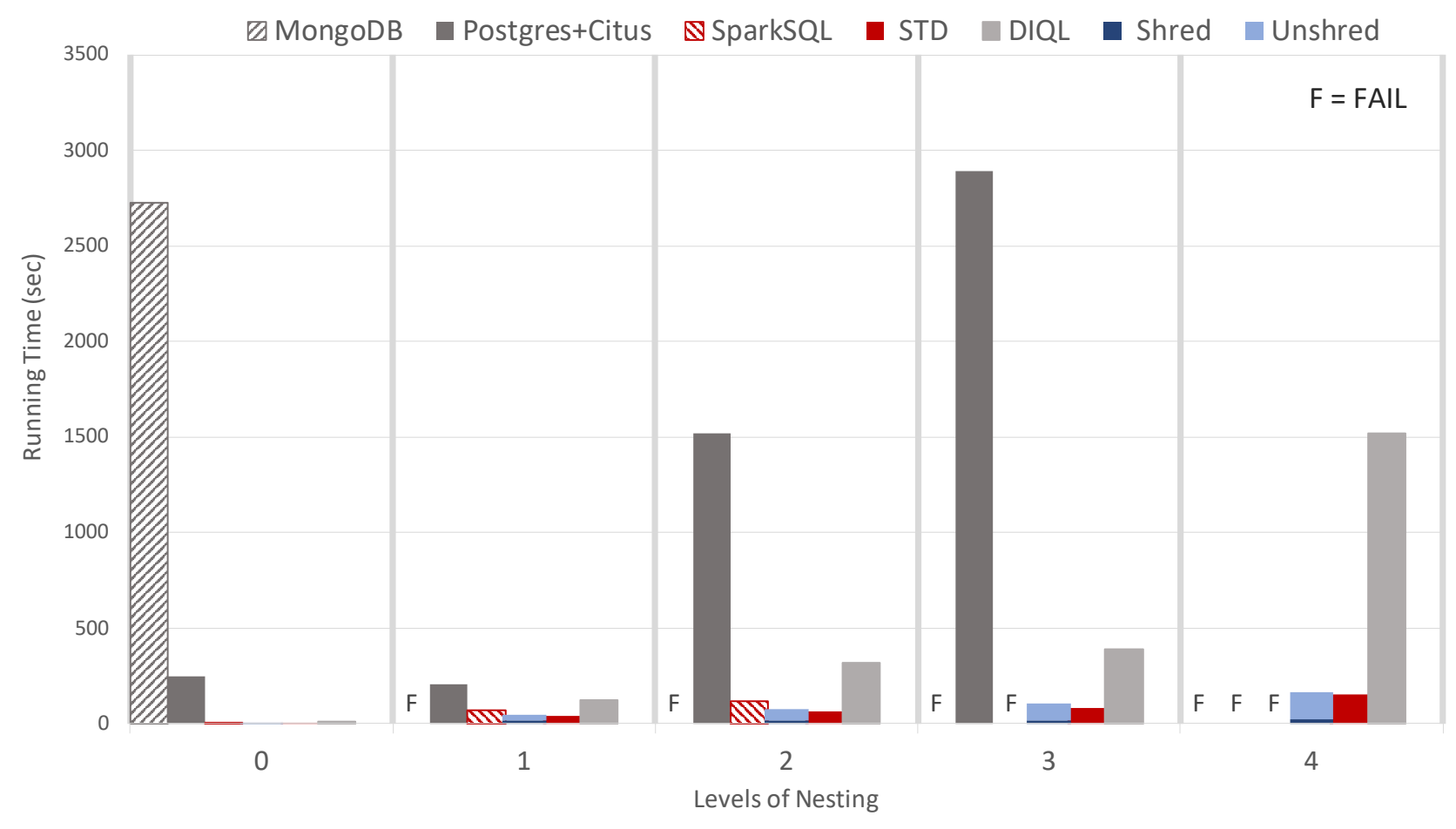

(a) Narrow schema

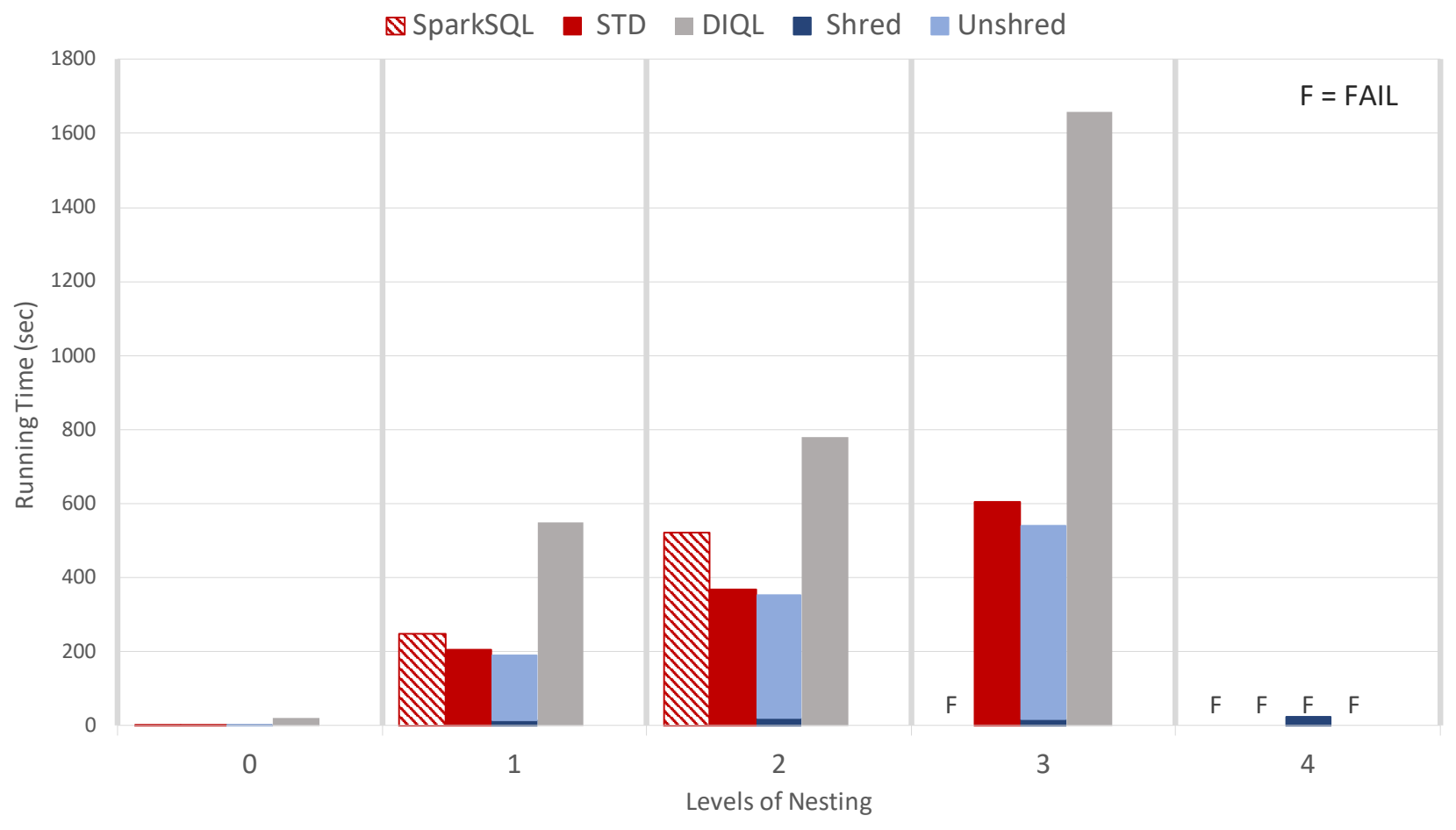

(b) Wide schema

Figure 15: Performance comparison of flat-to-nested queries including all competitors. 


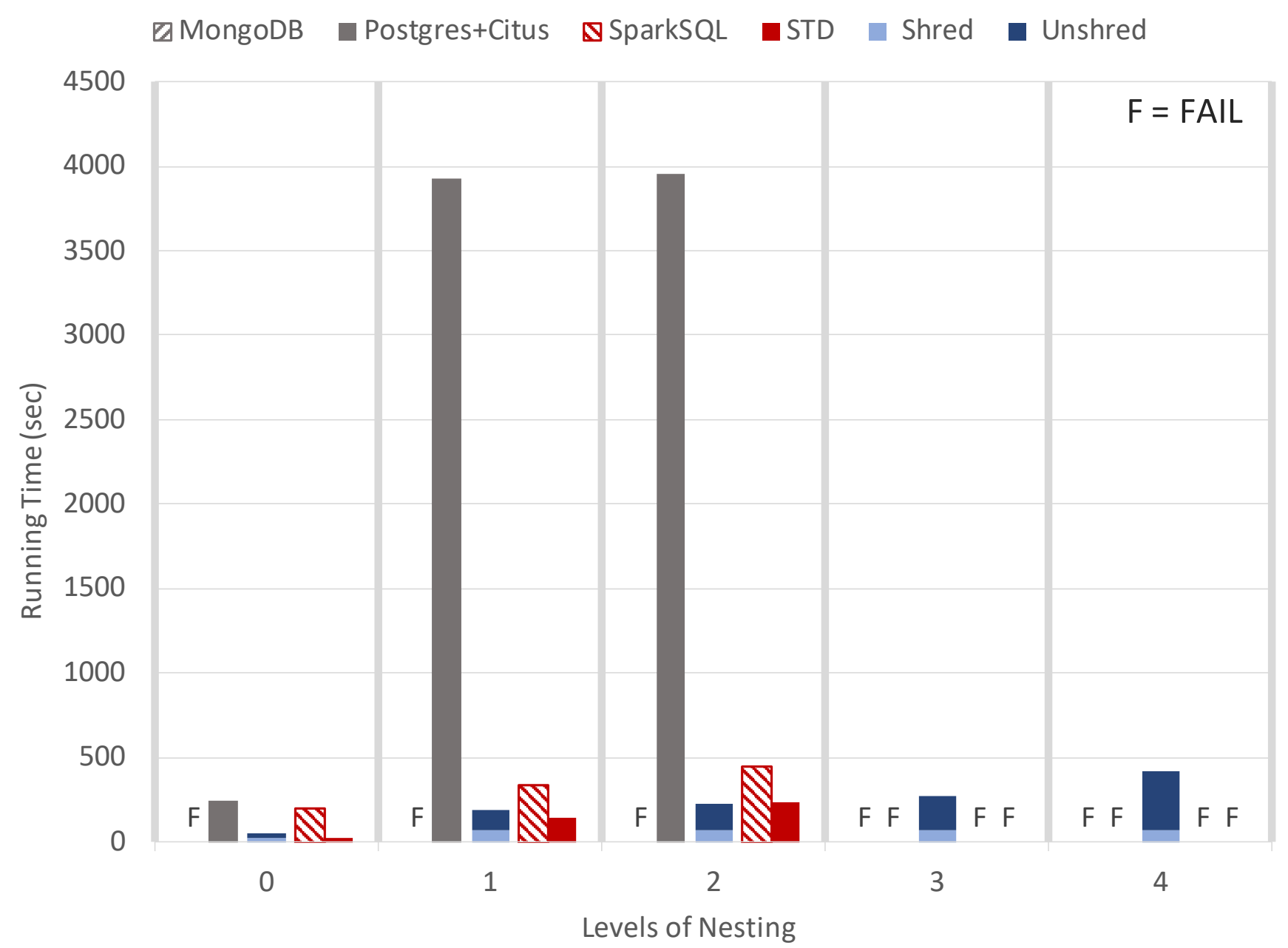

Figure 16: Performance comparison of narrow nested-to-nested queries including all competitors. 


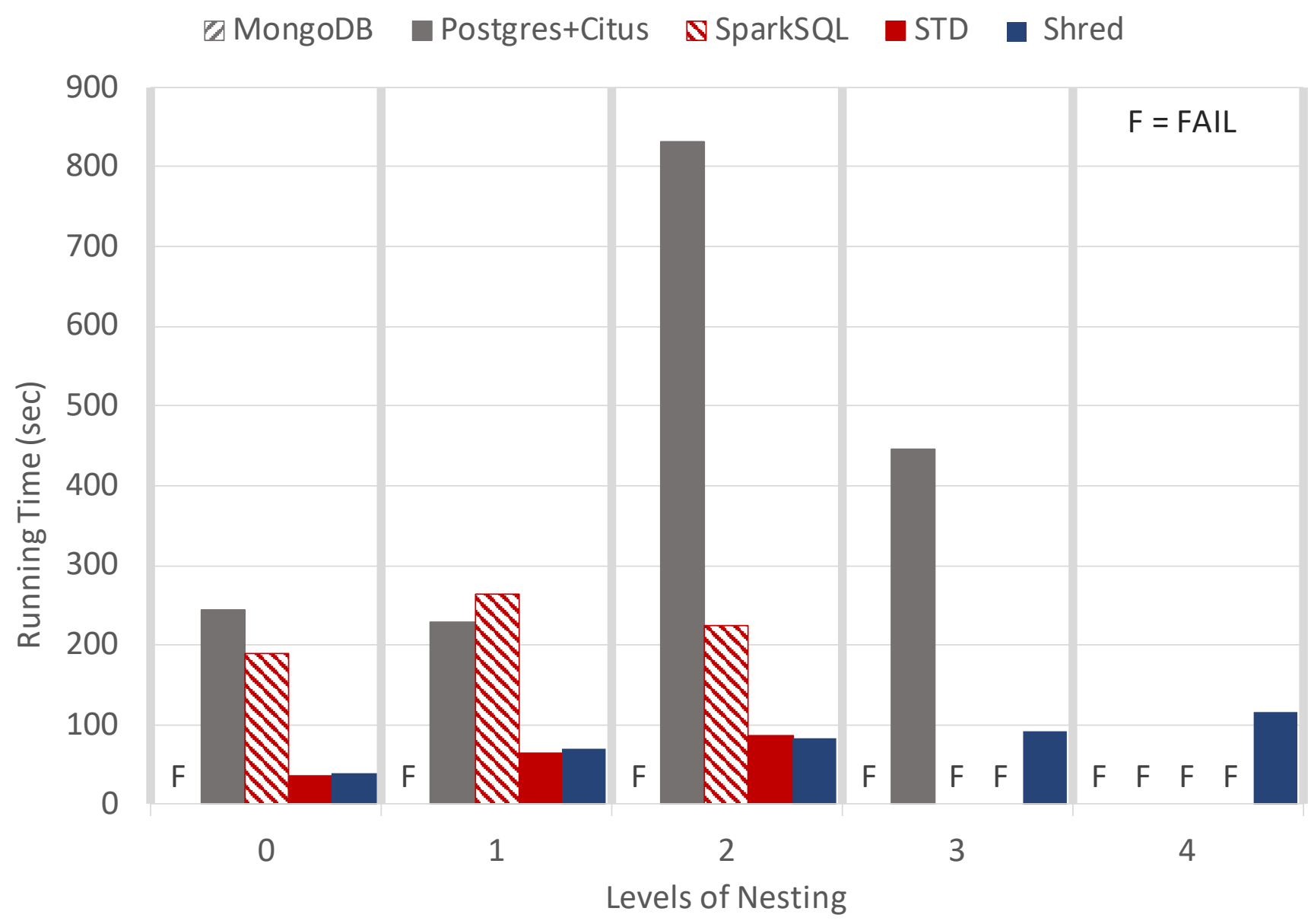

Figure 17: Performance comparison of narrow nested-to-flat queries including all competitors. 


\section{TIME (s) $\quad$ UNSHRED $\square$ SHRED $\quad$ STANDARD $\square$ SPARK-SQL}

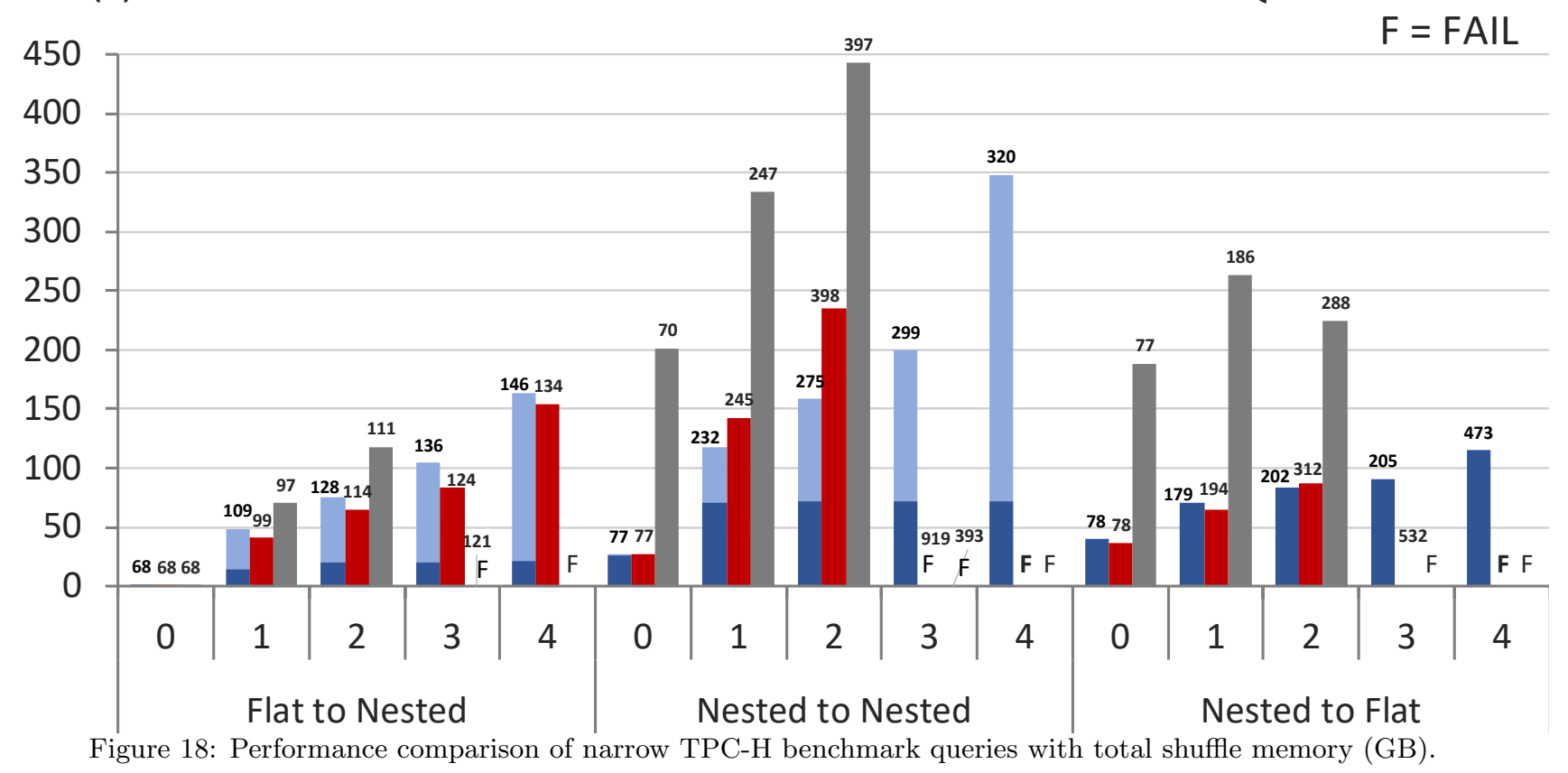

Nested-to-flat for non-skewed data. Figure 17 displays the results for MongoDB, Postgres Citus, SparkSQL, SHRED, UNSHRED and STANDARD for the narrow nested-to-flat queries of the TPC-H benchmark. As with the previous two query categories, we provide only the narrow variants for MongoDB and Postgres Citus. The wide variants for SparkSQL, SHRED, Unshred, and Standard can be found in the main body of the paper. Due to the poor performance of these systems, the nested inputs for MongoDB and Postgres Citus were preprocessed with projections pushed; thus, MongoDB and Postgres Citus take the materialized narrow flat-to-nested query as input. The other systems take the materialzied wide flat-to-nested query as input to better explore the effects of projection.

\section{E.3 Total shuffle for non-skewed TPC-H benchmark}

Figure 18 and Figure 19 provides annotated results for Figure 7 in Section 6 of paper, which includes the total shuffled memory (GB) for each run. If a job crashes at a particular nesting level, we do not report any further total shuffle memory. As above, MongoDB and Postgres Citus take the materialized narrow flat-to-nested query as input; whereas, the other methods take the materialized wide flat-to-nested query as input.

\section{E.4 Standard complation framework optimizations}

This experiment highlights how the framework can leverage database-style optimizations to automatically generate programs that are comparable to hand-optimized programs. Plans are generated using the standard compilation route with an increasing level of optimization applied to both the flat-to-nested and nested-to-nested queries. Figure 20 shows the results of this experiment. STANDARD (no opt) is the standard compilation route with no optimizations; this corresponds to the plan that comes directly from unnesting. Standard (pushed projections) is the standard compilation route with projections pushed. Standard applies all optimizations that produce the optimal plan, which is the plan in all experiments in the body of the paper; this includes pushed projections, nesting operators pushed past join operators, and merged into cogroups where relevant.

The results show that even simple optimizations like pushing projections can provide major performance benefits for flattening methods. For example, Figure 20a shows that projections have not only increased performance of the standard compilation route, but have allowed the strategy to survive to deeper levels of nesting. This is expected since the experiments in the previous sections have shown that the performance of STANDARD is heavily impacted by the presence of projections (ie. the number of attributes an output tuple). For nested-to-nested queries, STANDARD is the only strategy to survive past one level of nesting. These results show that database-style optimizations are not only beneficial to improve performance, but are necessary when using flattening methods even with shallow-nested objects. 


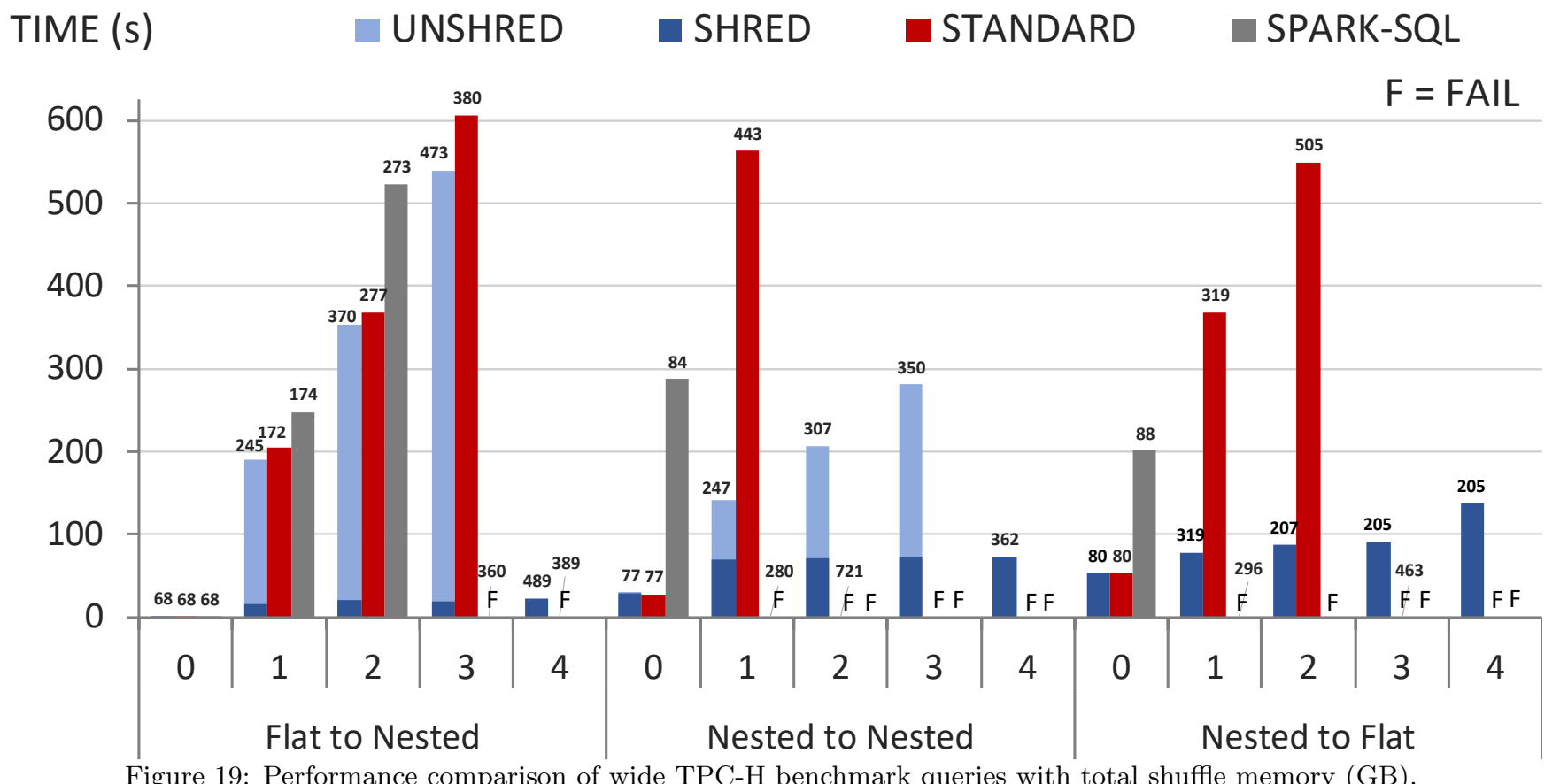

\section{E.5 COP shuffle in skew-handling results}

Figure 21 shows the amount of data shuffled from COP prior to the nested join with Part for the nested-to-nested TPC-H query used in the skew-handling results in the paper. The results highlight how the skew-aware shredded compilation route leads to less than a gigabyte of shuffling for moderate and high levels of skew. There are no COP shuffle results for the standard compilation route since query fails during execution while attempting to flatten COP. SparkSQL survives flattening for high levels of skew, but fails while performing the join with Part. The shuffling of the standard compilation route shows that at lower levels of skew the local aggregation is beneficial. At higher levels of skew the local aggregation reduces the data in the skew-handling variation of the compilation route to about $4.5 \mathrm{G}$; however, the skew-aware compilation route has reduced this to only megabytes of data leading to $74 \mathrm{x}$ less shuffle than the skew-unaware compilation route.

\section{E.6 Skew-handling results without aggregations pushed}

Figure 22 shows the runtimes for the standard and shredded compilation routes when aggregations are not pushed, for both the skew-aware and skew-unaware variants. The results show that without pushing aggregation, the skew-unaware methods decline in performance in comparison to the skew-unaware runs in the body of the paper (with aggregation pushed). These results highlight how pushing aggregation plays a key factor in the TPC-H skewed dataset to help with skewed data; the benefit in this case is a consequence of the TPC-H data generator, which duplicates values to create skew. We include these results to provide insight into how the skew-aware method could perform if aggregation pushing was not a key factor in the performance of the skew-unaware methods.

\section{E.7 Overhead of skew-handling}

Figure 23 shows the overhead of skew-handling for non-skewed data, comparing the results for the skew-unaware version of the compilation framework to the skew-aware version of the compilation framework for both the shredded and standard

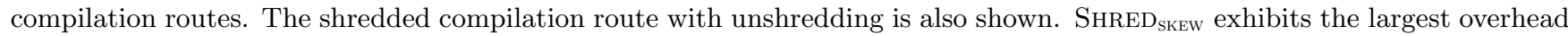
for heavy key collection. Both STANDARD SKEW $_{\text {and }}$ SHRED $_{\text {SKEW }}$ calculate heavy keys for Lineitem $\bowtie$ Part, which takes about $12 \mathrm{~s}$. The main overhead of $\mathrm{SHRED}_{\mathrm{SKEW}}$ is the heavy key calculation within the final casting of the lowest-level dictionary with BagToDict. Unshredding does no heavy key calculations, thus there is no additional overhead for SHRED SKEW $^{+U}$. 


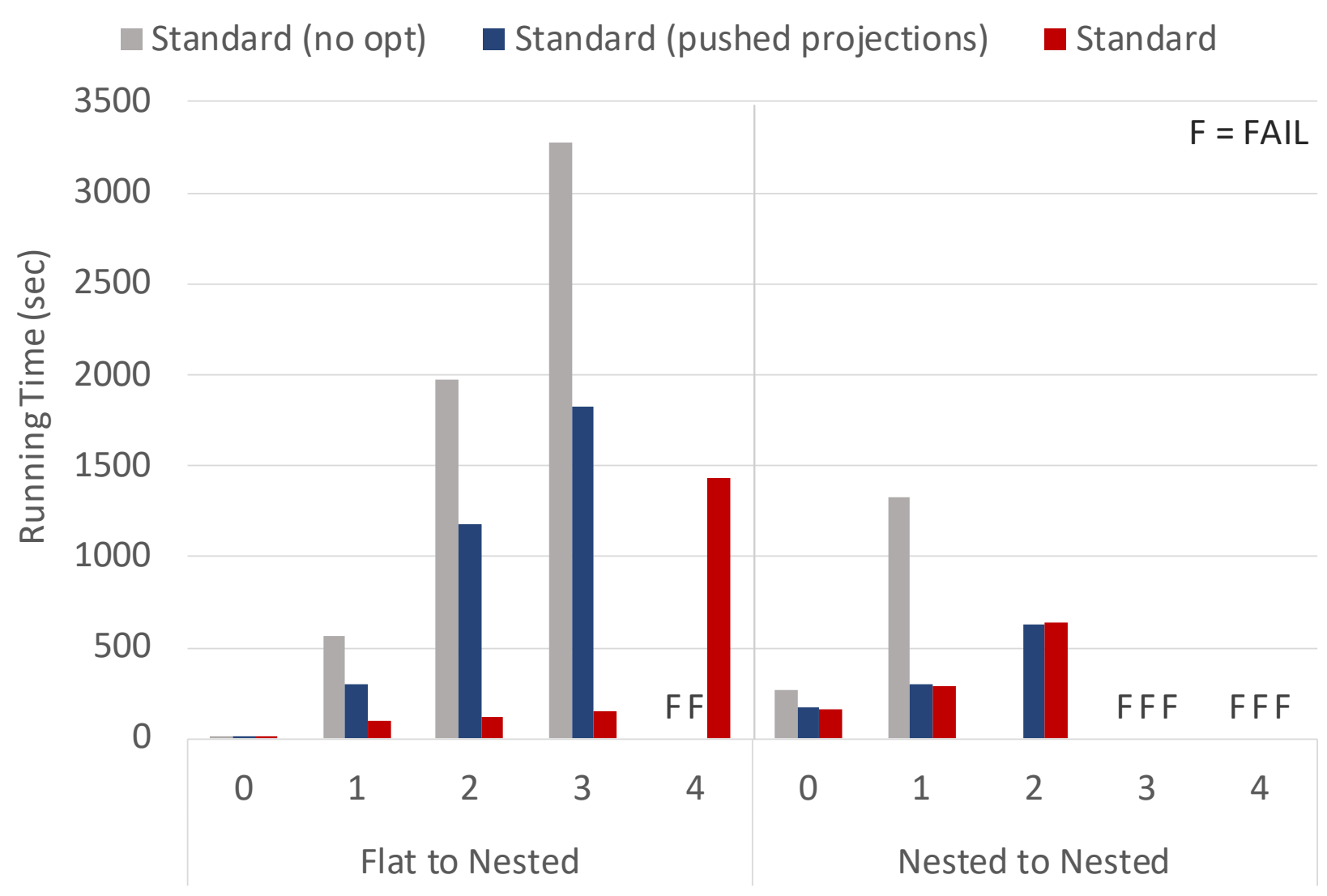

(a) Narrow schema

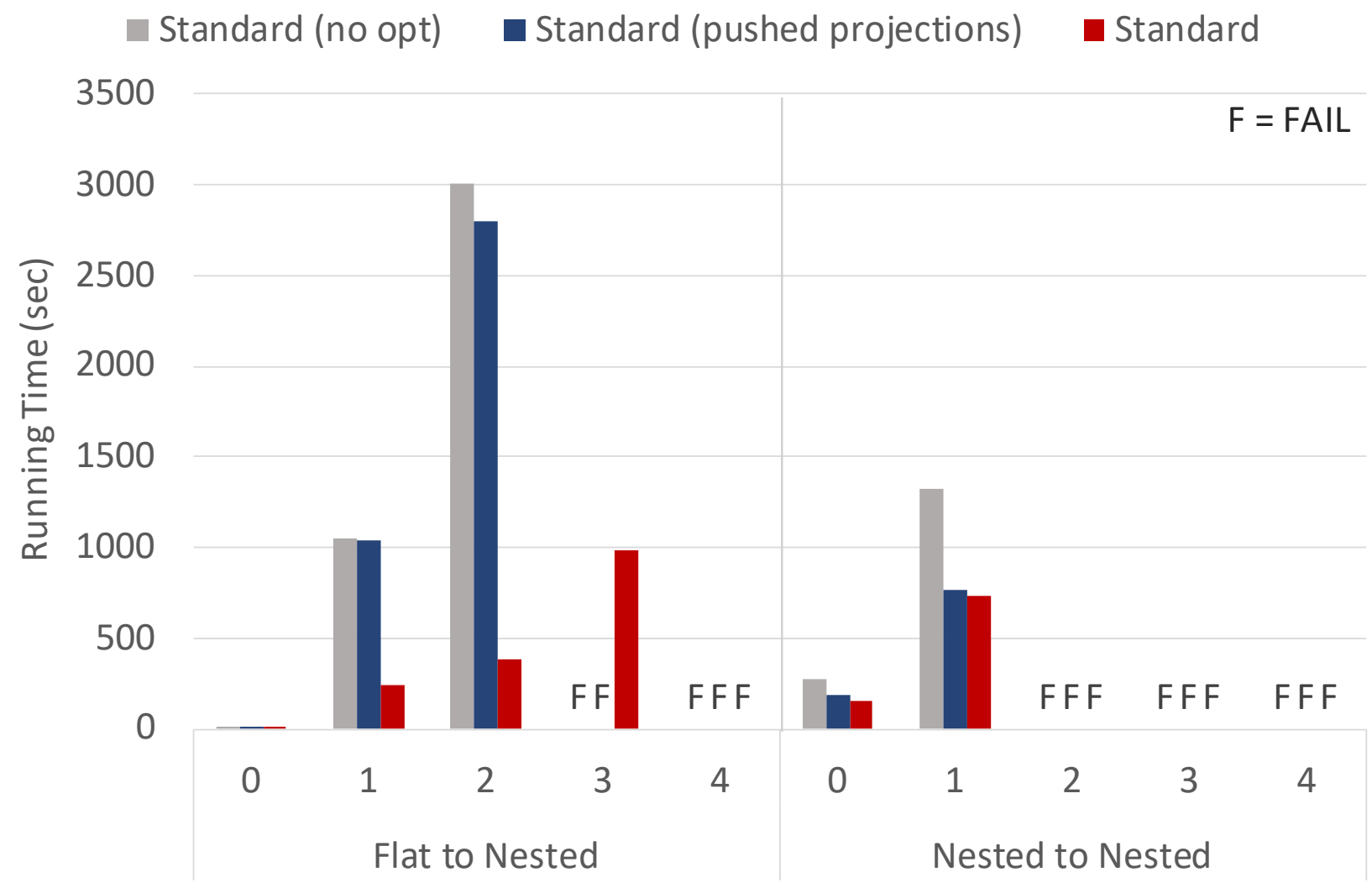

(b) Wide schema

Figure 20: Performance comparison of benchmarked queries for increasing optimization levels of the standard compilation route. 


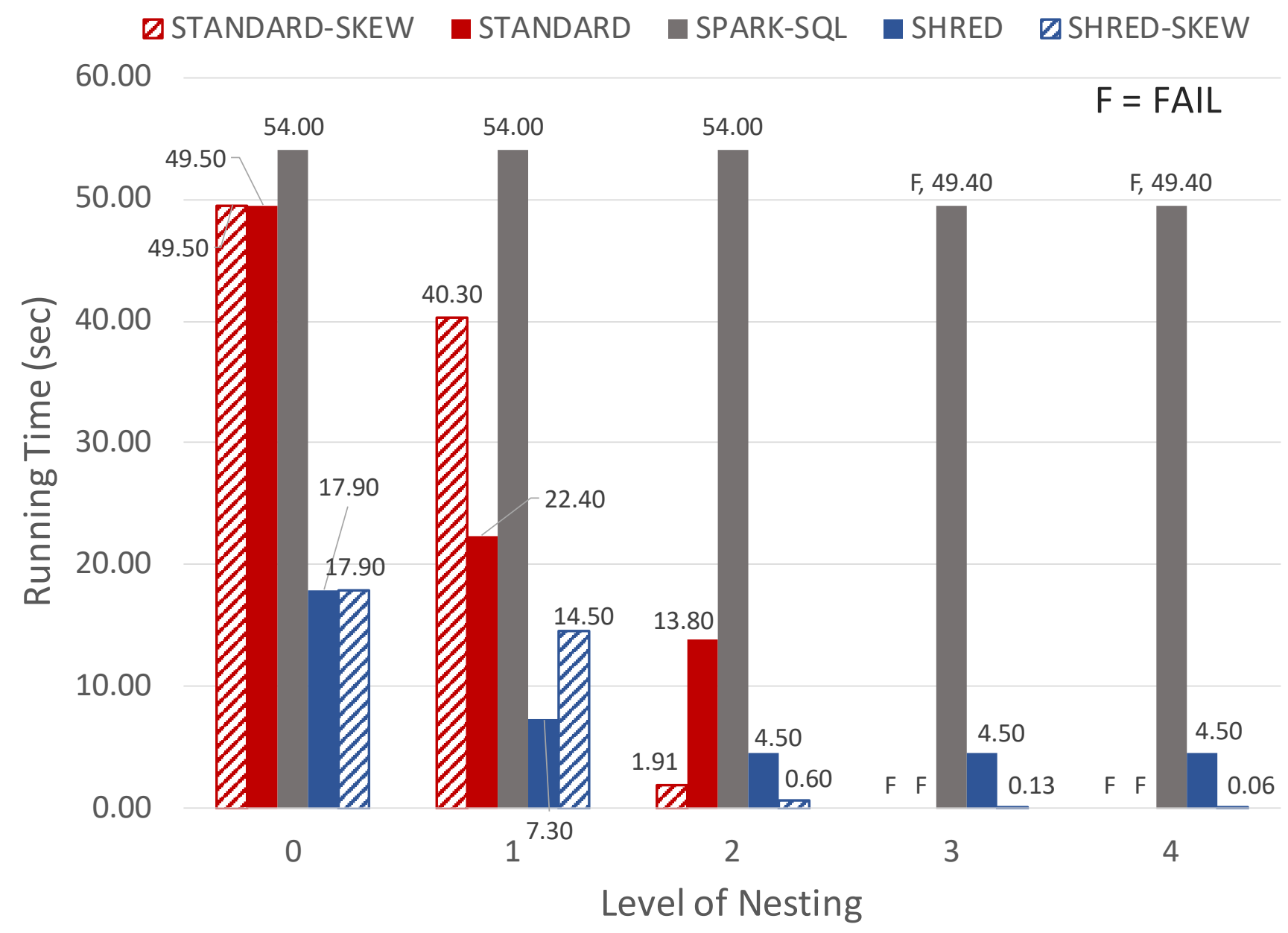

Figure 21: Amount of shuffled data from COP prior to joining with Part in level 2, narrow, nested-to-nested TPC-H query for skew-aware and skew-unaware variants, as well as SparkSQL. 


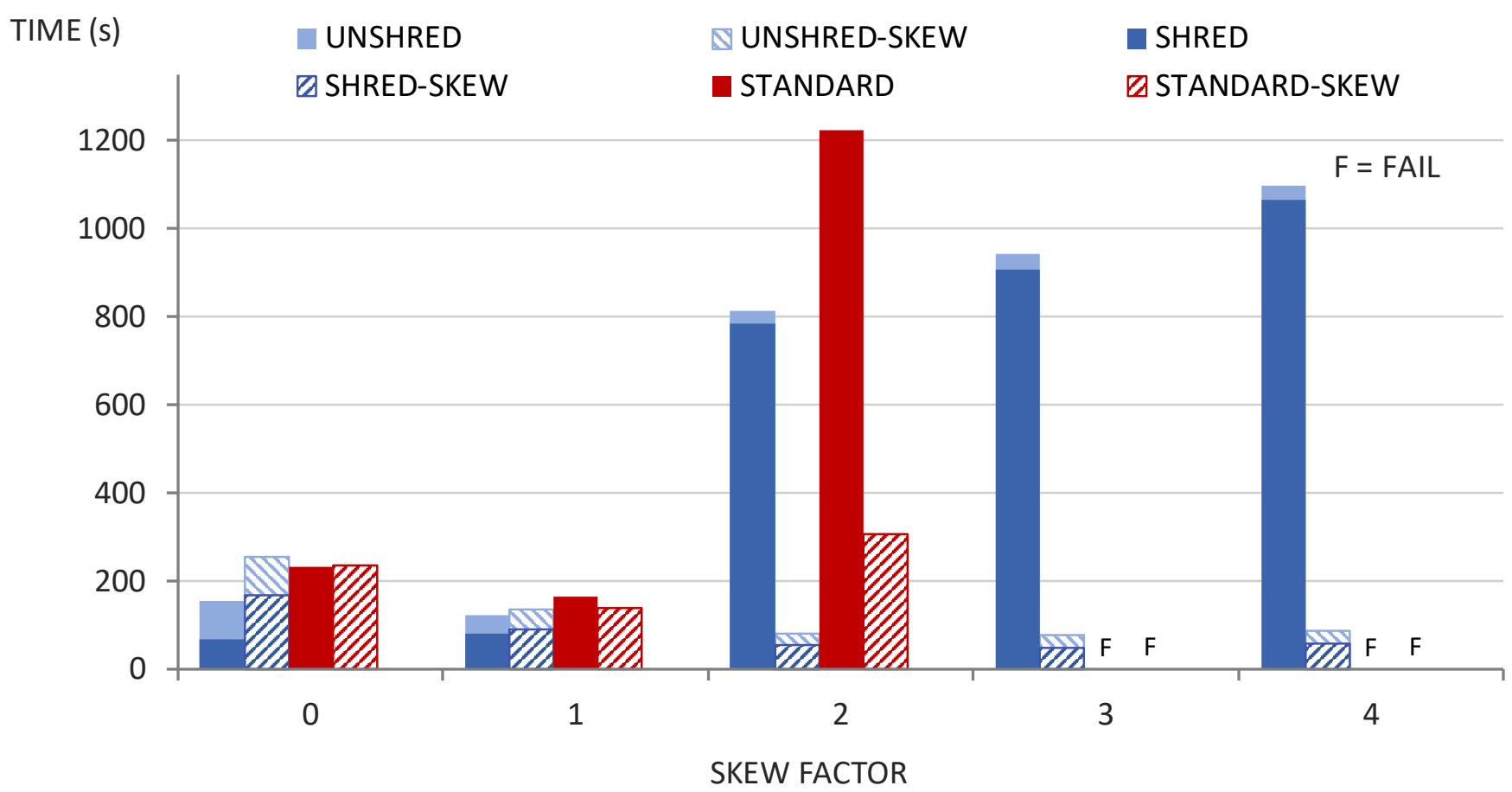

Figure 22: Performance comparison of skew-aware and non-skew aware standard and shredded compilation without aggregation pushing.

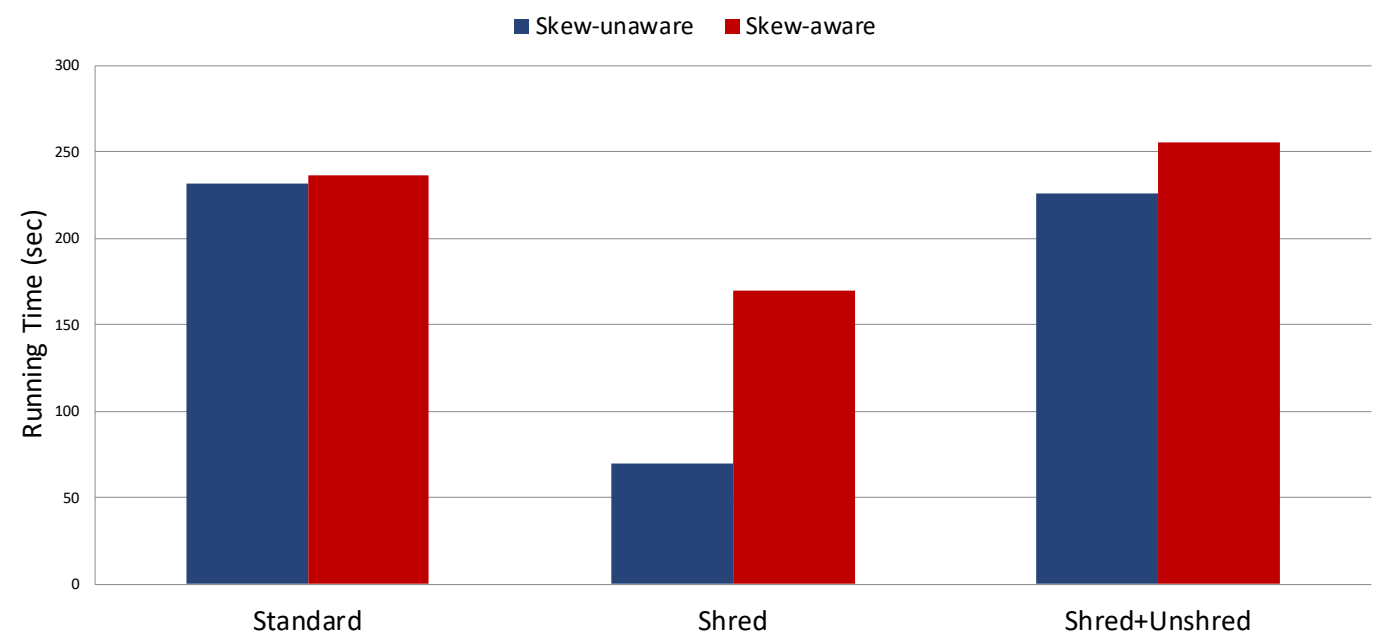

Figure 23: Skew-handling overhead for skew-unaware and skew-aware variants of the standard and shredded compilation routes. 